\title{
Trading and Returns under Periodic Market Closures
}

\author{
HARRISON HONG and JIANG WANG*
}

\begin{abstract}
This paper studies how market closures affect investors' trading policies and the resulting return-generating process. It shows that closures generate rich patterns of time variation in trading and returns, including those consistent with empirical findings: (1) U-shaped patterns in the mean and volatility of returns over trading periods, (2) higher trading activity around the close and open, (3) more volatile open-to-open returns than close-to-close returns, (4) higher returns over trading periods than over nontrading periods, (5) more volatile returns over trading periods than over nontrading periods. It also shows that closures can make prices more informative about future payoffs.
\end{abstract}

WE MODEL A COMPETITIVE STOCK MARKET with periodic closures in which investors trade for both allocational and informational reasons. We use the model to study how market closures intrinsically affect investors' trading behavior and the return-generating process. The purpose of this analysis is to increase our understanding of the time variation in security trading and returns that are associated with regular market closures, such as the intraday and intraweek patterns in stock returns, volatility, and trading volume.

We consider a stock market in which the exogenous information flow is homogeneous over time and the market closes periodically. When the market is open, investors trade the stock either to rebalance their overall portfolio of assets, which also includes other illiquid assets, or to speculate on future stock payoffs using their private information. In particular, investors adjust their asset portfolio by trading the stock in order to hedge the risk of illiquid assets. We refer to these trades as hedging trades and those motivated by private information as speculative trades. When the market is closed,

\footnotetext{
* Hong is from the Graduate School of Business, Stanford University, and Wang is from the Sloan School of Management, Massachusetts Institute of Technology, and NBER. The authors thank Jennifer Huang for programming assistance and an anonymous referee for many valuable suggestions. They also thank Glenn Ellison, John Heaton, Craig Holden, Andrew Lo, Steve Slezak, Jeremy Stein, René Stulz (the editor), the NBER Asset Pricing Lunch Group, and participants of seminars at the London School of Economics, New York University, Princeton University, the University of California at Los Angeles, the University of Houston, the University of Illinois, the University of Pennsylvania, the 1995 WFA meetings, and the Indiana University Symposium on the Organization of Financial Trade and Exchange Mechanism for comments. Hong acknowledges support from an NSF Fellowship and Wang acknowledges support from a Batterymarch Fellowship, NSF grant SES-9414112, and the Laboratory for Financial Engineering at MIT.
} 
investors hold on to their closing positions from the previous trading period despite their desire to trade as new information arrives. Consequently, investors optimally adjust their trading strategies during the trading period (in anticipation of and following market closures), which gives rise to time variations in equilibrium returns.

Market closures impact the economy in two ways: they preclude investors from trading in the market, and they prevent investors from learning about the economy by observing market prices and trading activities. The lack of trading increases the risk of holding the stock over closures, causing investors to reduce their hedging trade at the market close. The anticipated decrease in investors' hedging trade tends to make the stock price decrease over time as a larger premium is demanded on the stock, and tends to make the stock price less sensitive to investors' hedging needs. The lack of market prices as a source of information gives rise to time variation in the information asymmetry among investors. While information asymmetry increases during the closure, it often decreases as trading continues after the market reopens. The decrease in information asymmetry tends to make the stock price increase as a smaller premium is demanded on the stock, and tends to make the stock price more sensitive to investors' private information on future payoffs as more private information is impounded into the price.

The actual time variation in the stock price is determined by the interaction of these two effects: the effect of time-varying hedging trade and the effect of time-varying information asymmetry. The interaction between these two effects can generate a rich set of patterns in stock returns. For example, when the effect of time-varying hedging trade dominates, both the mean and volatility of stock returns decrease over time during the trading periods. When the effect of time-varying information asymmetry dominates, both the mean and volatility increase over time. For some parameter values, the effect of time-varying hedging demand dominates around market open and the effect of time-varying information asymmetry dominates around market close. In this case, both the mean return and return volatility are U-shaped during the trading periods, higher around the open and close and lower during midperiod.

Additionally, when decreasing information asymmetry causes the stock price to increase during the day, the return over trading periods is higher than the return over nontrading periods. Also, trading reveals investors' private information, which moves the price; hence returns over the trading periods tend to be more volatile than returns over the nontrading periods. Furthermore, the information accumulation during a market closure gives rise to high trading volume at the open, and the reduction in investors' hedging positions at the end of a trading period can give rise to high trading volume at the close.

The interaction between investors' hedging trade and the market's information flow also gives rise to other interesting phenomena. For example, as investors adjust their hedging trade in anticipation of future market closures, the endogenous information flow in the economy also changes. As the level of hedging trade decreases at the close, price changes are more likely 
to be caused by speculative trade, and hence are more informative about investors' private information on future payoffs. As a result, closures can make market prices more informative about stock payoffs by reducing the level of "noise" in stock prices from hedging trade.

There is an extensive literature on the empirical patterns of stock returns and trading activities associated with market closures. These patterns include the following.

a. Intraday mean return and volatility are U-shaped. ${ }^{1}$

b. Intraday trading volume is U-shaped. ${ }^{2}$

c. Open-to-open returns are more volatile than close-to-close returns. ${ }^{3}$

d. Weekend returns are lower than weekday returns. ${ }^{4}$

e. Returns over trading periods are more volatile than returns over nontrading periods. ${ }^{5}$

Many of these patterns are robust with respect to different market microstructures (such as the NYSE, Nasdaq, and the interbank market of currencies).

The observed patterns have generated strong interest in developing theoretical models to understand them. Admati and Pfleiderer $(1988,1989)$ and Foster and Viswanathan $(1990,1993)$ analyze how investors' discretion in timing their liquidity trade can lead to endogenous concentration of trades and price changes. Their models, however, leave the actual timing of concentration in trade and price changes undetermined. Additional assumptions on the time-variation of liquidity trade and/or information arrival are needed in order to produce the empirical patterns that are punctuated by closures. Brock and Kleidon (1992) point out the link between time-variation in market activity and closures. They solve for investors' optimal marketmaking policies under periodic market closures. Unfortunately, their analysis is in a partial equilibrium setting and cannot speak to the equilibrium patterns in return and trading. Using a noisy rational expectations equilibrium setting, Slezak (1994) examines the impact of closure on equilibrium returns by comparing two equilibria, the one with closure and the other without closure. The difference in prices between these two cases suggests that market closure may help to explain some of the observed patterns.

Slezak's (1994) results, however, depend crucially on the exogenous specification of the liquidity trade. Under the assumption that the liquidity trade is constant over time, the higher risk (thus lower liquidity) before and after a closure leads to higher price volatility. If investors can instead allocate

\footnotetext{
${ }^{1}$ See, for example, Andersen and Bollerslev (1997), Gerety and Mulherin (1994), Harris (1986, 1988, 1989), Kleidon and Werner (1996), Lockwood and Linn (1990), Rogalski (1984), Smirlock and Starks (1986), and Wood, McInish, and Ord (1985).

${ }^{2}$ See, for example, Chan et al. (1996) and Jain and Joh (1988).

${ }^{3}$ See, for example, Amihud and Mendelson (1987, 1991), Cao, Choe, and Hatheway (1995), Chan et al. (1996), and Stoll and Whaley (1990).

${ }^{4}$ See, for example, French (1980), Gibbons and Hess (1981), and Keim and Stambaugh (1984).

${ }^{5}$ See, for example, Amihud and Mendelson (1991), Barclay, Litzenberger, and Warner (1990), Fama (1965), French and Roll (1986), and Oldfield and Rogalski (1980).
} 
their trade accordingly, they may actually cut back their liquidity trade at the close, causing price volatility to decrease, not increase. Moreover, the intuition from comparing two different equilibria (with and without closure), though helpful, is inconclusive in explaining the actual time patterns. After all, these patterns appear in a single, intertemporal equilibrium, and hence need to be derived as the outcome of such an equilibrium.

Our model differs from existing models in several dimensions. First, we explicitly model investors' allocational and informational trades simultaneously. This stands in contrast to the noisy rational expectations models in the literature, in which allocational trade (liquidity trade) is exogenously specified. As we show in the paper, the fact that investors can choose the timing and size of their allocational trades is important in understanding the equilibrium return and trading patterns. ${ }^{6}$ Second, we focus on how market closures intrinsically affect investors' trading behavior and the return-generating process. In particular, we examine how closures generate endogenous time-variation in trading activity, information arrival, and price movements. Third, we derive the time patterns of return and trading activity from a single intertemporal equilibrium, which is different from Slezak (1994) who relies on a comparative static analysis of stationary equilibria. ${ }^{7}$ Fourth, we assume a competitive market and avoid strategic and market microstructure issues. By analyzing periodic market closures, we focus on those effects on equilibrium returns and trading activities that are solely associated with closures. We show that only periodic market closures are needed to qualitatively generate all the empirical patterns mentioned above.

The paper proceeds as follows. Section I describes the model. Section II defines the notion of equilibrium and Section III discusses the general solution of equilibrium. Section IV analyzes how market closures affect investors' hedging trade and equilibrium returns. Section V further examines the effect of information asymmetry on trading activity and stock returns. Section VI concludes. The Appendixes provides technical details and proofs.

${ }^{6}$ Admati and Pfleiderer $(1988,1989)$ and Foster and Viswanathan $(1990,1993)$ allow some of the liquidity traders to time their trade of given sizes. But they do not provide a complete justification for the behavior of liquidity traders. For example, trade sizes are exogenously specified and not all investors can time their trade. See also Spiegel and Subrahmanyan (1995).

${ }^{7}$ Our model differs from Slezak (1994) in several other ways as well. In our model, two things are present during market closures: new information (both public and private) on asset payoffs and shocks to investors' allocational demand for stock. In Slezak's model, only the former is present. Thus, in the absence of any new information on stock payoffs (public and/or private), investors still desire to trade during a closure in our model but not in Slezak's model. In other words, in the absence of new information on stock payoffs, a closure in our model still affects the equilibrium, but a closure in Slezak's model does not. In this sense, the closure in Slezak's model is more like a pure information event such as a news arrival. Furthermore, in our model, the repeated nature of closures is important in generating the return patterns. In particular, what happens around the market close is related to past and future openings of the market, and what happens around the open is related to closures in the past and future. In Slezak (1994), there is a single closure, the open is not affected by the future close, and the close is not affected by past open. 


\section{The Model}

We consider an economy defined on a continuous time-horizon $[0, \infty)$ with a single commodity, which is also used as the numéraire. The underlying uncertainty is characterized by an $n$-dimensional standard Wiener process $\boldsymbol{w}_{t}, t \in[0, \infty)$. There are two classes of investors denoted by $i=1,2$. Investors are identical within each class, but different across classes in endowments and private information. Let the population weight of the two classes of investors be $\omega$ and $1-\omega$, respectively, where $\omega \in[0,1]$. For convenience, we also refer to any investor in class $i$ as investor $i$. The economy is further defined below.

\section{A. Investment Opportunities}

Investors can invest in publicly traded securities or in private investment opportunities. Publicly traded securities include a risk-free money-market account and a risky stock. Private investment opportunities are linear production technologies available only to individual investors. The payoffs of these investments are as follows:

a. The money-market account pays a positive, constant rate of return $r$.

b. Each share of the stock pays a cumulative dividend $D_{t}$ where

$$
\begin{aligned}
& D_{t}=\int_{0}^{t}\left(G_{s} d s+\boldsymbol{b}_{D} d \boldsymbol{w}_{s}\right) \\
& G_{t}=G_{0}+\int_{0}^{t}\left(-a_{G} G_{s} d t+\boldsymbol{b}_{G} d \boldsymbol{w}_{s}\right) .
\end{aligned}
$$

c. Investor $i$ 's private technology yields a cumulative excess rate of return $q_{i, t}$, where

$$
\begin{aligned}
& q_{i, t}=\int_{0}^{t}\left(Y_{i, s} d s+\boldsymbol{b}_{q} d \boldsymbol{w}_{s}\right) \\
& Y_{i, t}=Y_{i, 0}+\int_{0}^{t}\left(-a_{Y} Y_{i, s} d s+\boldsymbol{b}_{i} d \boldsymbol{w}_{s}\right) .
\end{aligned}
$$

Here, $i=1,2, a_{G}, a_{Y}$ are positive constants, $\boldsymbol{b}_{D}, \boldsymbol{b}_{G}, \boldsymbol{b}_{q}, \boldsymbol{b}_{1}, \boldsymbol{b}_{2}$ are constant matrices of proper order. ${ }^{8} G_{t}$ gives the expected dividend growth of the stock at $t$, and $Y_{1, t}, Y_{2, t}$ give, respectively, the expected excess rate of return on class 1 and class 2 investors' private technologies. They all follow Gaussian AR(1) processes. The system $\left\{G_{t}, Y_{1, t}, Y_{2, t}\right\}$ then follows a Gaussian Markov

\footnotetext{
${ }^{8}$ Throughout this paper, we use the following conventions: (1) Bold-face letters denote matrices (or vectors). (2) Given a set of elements $e_{1}, e_{2}, \ldots, e_{m}$ (of proper order), $\left[e_{1} ; e_{2} ; \ldots ; e_{m}\right]$ denotes the column matrix and $\left[e_{1}, e_{2}, \ldots, e_{m}\right]$ the row matrix from these elements. (3) 1 denotes an identity matrix and $\mathbf{0}$ a zero matrix of proper order.
} 


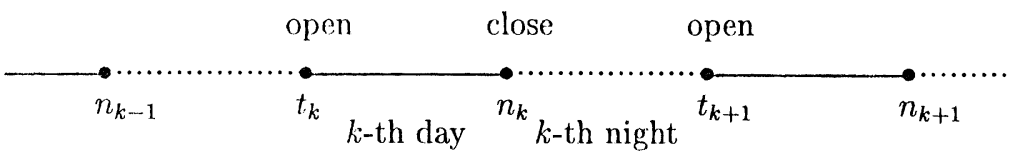

Figure 1. Time line. Intervals of solid lines represent periods when the stock market is open and intervals of dotted lines represent periods when the stock market is closed.

process, which completely determines the distribution of future payoffs on the stock and the investors' private technologies. ${ }^{9}$ For future convenience, let $\boldsymbol{Z}_{t}=\left[G_{t}, Y_{1, t}, Y_{2, t}\right]^{\prime}$. Then

$$
\boldsymbol{Z}_{t}=\boldsymbol{Z}_{0}+\int_{0}^{t}\left(-\boldsymbol{a}_{Z} \boldsymbol{Z}_{s} d s+\boldsymbol{b}_{Z} d \boldsymbol{w}_{s}\right)
$$

where $\boldsymbol{Z}_{0}=\left[G_{0} ; Y_{1,0} ; Y_{2,0}\right], \boldsymbol{a}_{Z}=\left[\left[a_{G}, 0,0\right] ;\left[0, a_{Y}, 0\right] ;\left[0,0, a_{Y}\right]\right]$, and $\boldsymbol{b}_{Z}=$ $\left[\boldsymbol{b}_{G} ; \boldsymbol{b}_{1} ; \boldsymbol{b}_{2}\right]$. Investors can continuously invest in their private technologies. They can also trade the securities in a competitive securities market when the market is open. ${ }^{10}$ The money market is open continuously but the stock market is open only periodically. Thus, investors can adjust their moneymarket account at any time, but they can trade the stock only when the stock market is open. The dividend and interest payments are received continuously as they accrue. $P_{t}$ denotes the share price of the stock when the stock market is open.

Figure 1 shows the time sequence of events. Here, $\left[t_{k}, n_{k}\right]$ is the $k$-th (stock) trading period, and $\left(n_{k}, t_{k+1}\right)$ is the $k$-th nontrading period, where $k=0,1,2, \ldots$ We further require that the lengths of the trading period $(T)$ and nontrading period $(N)$ are constant over time. That is, $n_{k}-t_{k}=T$ and $t_{k+1}-n_{k}=N$ for all $k$. Consequently, $t_{k}=k(T+N)$ and $n_{k}=t_{k}+T$. When $N=0$, the economy reduces to the case of the stock market being permanently open. When $T=$ 0 , it reduces to the discrete-trading case. Further, let $\mathcal{T}_{k}=\left[t_{k}, n_{k}\right]$ denote the $k$-th trading period, $\mathcal{N}_{k}=\left(n_{k}, t_{k+1}\right)$ the $k$-th nontrading period, $\mathcal{T}=\cup_{k}\left[t_{k}, n_{k}\right]$ the set of times the stock market is open, and $\mathcal{N}=\cup_{k}\left(n_{k}, t_{k+1}\right)$ the set of times the stock market is closed. For convenience, we refer to the periods when the stock market is open and closed as "day" and "night" respectively.

\footnotetext{
${ }^{9}$ Instantaneous returns on the private technologies of all investors are assumed to be perfectly positively correlated. Our results stay qualitatively the same if partial correlation is allowed.

${ }^{10}$ The competitive assumption can be justified by the existence of many (possibly an infinite number of) identical investors within each class. Furthermore, for each class of investors, we can construct a representative investor who has the same information, preference, and investment opportunities as each individual investor, but has the total endowments of all investors in the class. The risk tolerance of this representative investor is the sum of the risk tolerance of individual investors in the class (see, e.g., Rubinstein (1974)). The economy then can be viewed as consisting of the two representative investors, interacting competitively in the securities market.
} 


\section{B. Information Distribution}

All investors observe realized dividends and market prices of the stock. Class 1 investors observe all the state variables governing the investment opportunities in the economy $\left(G_{t}, Y_{1, t}\right.$, and $\left.Y_{2, t}\right)$, but class 2 investors only observe the state variable governing their own investment opportunities $\left(Y_{2, t}\right)$. Additionally, there is a set of public signals denoted by $\boldsymbol{U}_{t}$ where

$$
\boldsymbol{U}_{t}=\boldsymbol{U}_{0}+\int_{0}^{t}\left(\boldsymbol{a}_{U} \boldsymbol{Z}_{s} d t+\boldsymbol{b}_{U} d \boldsymbol{w}_{s}\right)
$$

$\boldsymbol{U}_{0}$ is jointly normal with $G_{0}, Y_{1,0}$, and $Y_{2,0}$, and $\boldsymbol{a}_{U}, \boldsymbol{b}_{U}$ are constant matrices of proper order. Thus, class 1 investors have superior information about future stock payoffs. Let $\mathcal{I}_{i, t}$ denote the information set of investor $i$ at time $t(t \geq 0), i=1,2$. We have $\mathcal{I}_{1, t}=\left\{\boldsymbol{U}_{s}, P_{s}, D_{s}, G_{s}, Y_{1, s}, Y_{2, s}, q_{1, s}: 0 \leq s \leq t\right\}$ and $\mathcal{I}_{2, t}=\left\{\boldsymbol{U}_{s}, P_{s}, D_{s}, Y_{2, s}, q_{2, s}: 0 \leq s \leq t\right\}$, where $P_{t}$ is only defined for $t \in \mathcal{T}$. $\mathcal{I}_{t}=$ $\mathcal{I}_{1, t} \vee \mathcal{I}_{2, t}$ then gives the full information set about the economy and $\mathcal{I}_{i, t} \subseteq$ $\mathcal{I}_{t} \forall i=1,2$.

\section{Endowments, Policies, and Preferences}

In addition to his private technology, each investor is also endowed with one share of the stock. He chooses investment and consumption policies to maximize the expected utility over his lifetime consumption. For investor $i$ $(i=1,2)$, let $c_{i, t}$ be his consumption at $t, y_{i, t}$ his investment in the private technology, and $\theta_{i, t}$ the number of stock shares he holds. Then, $\left\{c_{i, t}: t \in\right.$ $[0, \infty)\}$ and $\left\{y_{i, t}: t \in[0, \infty)\right\}$ give his consumption policy and production policy, respectively, and $\left\{\theta_{i, t}: t \in \mathcal{T}\right\}$ his stock trading policy, defined only when the stock market is open. In short, we use $\left\{c_{i, t}, y_{i, t}, \theta_{i, t}\right\}$ to denote investor $i$ 's policy, which is adapted to his information $\mathcal{I}_{i, t}$. We further restrict investors' trading and production policies to predictable, square-integrable processes (with respect to the return processes) and the consumption policy to integrable processes. ${ }^{11}$

We assume that all investors have expected utility of the following form:

$$
\mathrm{E}\left[-\int_{t}^{\infty} e^{-\rho(s-t)-\gamma c_{i, s}} d s \mid \mathcal{I}_{i, t}\right] \quad(i=1,2),
$$

where $\rho$ and $\gamma$ (both positive) are the time-discount coefficient and the riskaversion coefficient, respectively.

${ }^{11}$ The notion of integrability here refers to integrability over any finite period $[0, t] \forall t \geq 0$. See, for example, Harrison and Pliska (1981) for a discussion on the need of integrability conditions. 


\section{Comments on the Model}

Many features of our model, such as the dividend process, the interest rate, investor preferences, and information structure, resemble those in the models of Campbell and Kyle (1993) (under homogeneous information) and Wang (1993, 1994) (under heterogeneous information). Gaussian distributions for dividends and returns on nontraded assets, a constant interest rate, and the constant absolute risk aversion for investor preferences are adopted here merely for tractability.

In our model, investors trade the stock for two reasons: hedging and speculation. First, an investor's portfolio consists of holdings of traded securities (the stock and money-market account) and his private investment. When the returns to his private investment and the stock are correlated, an investor's stock holding depends not only on the expected return on the stock itself but also on the expected return on his private investment. In particular, each investor uses the stock to hedge the risk from his private investment. As an investor adjusts his private investment in response to changes in its expected return, he also revises his hedging position in the stock. This generates the hedging trades in the market. Second, some investors have private information about future stock payoffs. Those investors take speculative positions based on their private information in anticipation of future profits. This generates the speculative trades in the market.

In the model, private investments take the form of investing in private technologies. This particular form of private investment, if taken literally, might seem somewhat artificial, especially when the model is used to explain return behavior over short time-intervals (such as intraday or intraweek). However, we can reinterpret these private investments as illiquid assets that investors hold (see, e.g., Huang and Wang (1997)). It is conceivable that the value of these illiquid assets can change as quickly as the value of traded assets. ${ }^{12}$ The distinction between private technologies and illiquid assets is that an investor can adjust the level of investment in the former case but not in the latter. But this distinction is unimportant for our purpose. Since resources are always available via borrowing, investors trade in the stock not to get resources to invest, but to control portfolio risk. Thus, to the extent that trade motivated by private technology shocks mirrors trade behavior generated by illiquid assets, our model with private technologies captures a salient feature of real markets. We choose the setting with private technologies merely for the simplicity in its solution. More generally, what we need here is a motive for investors to trade for allocational reasons. As will become clear through our discussion, how this motive is generated in the model is not crucial for our main results.

\footnotetext{
${ }^{12}$ For example, consider an investor holding both U.S. and Japanese stocks. When the U.S. market is open, the Japanese market is closed and his holding of the Japanese stocks becomes illiquid. Any news on Japanese stocks may cause him to trade the U.S. stocks in order to rebalance his overall portfolio.
} 
Even though closures are modeled here as periods when the market physically shuts down, they can be broadly interpreted as periods when a subset of investors withdraws from the market. The qualitative nature of the model is not changed if the market is assumed to be open all the time but most investors stop trading in the night. The lack of sufficient market participation makes trading costly and prices uninformative. A market shutdown is simply the extreme of this situation..$^{13}$

\section{E. Distributional Assumptions}

In order to be more specific in our analysis, we make several simplifying assumptions on the distribution of underlying shocks. In particular, we assume that

$$
\boldsymbol{w}_{t}=\left[w_{D, t} ; w_{G, t} ; w_{1, t} ; w_{2, t} ; w_{q, t} ; \boldsymbol{w}_{U, t}\right]
$$

and

$$
\begin{aligned}
& \boldsymbol{b}_{D}=\sigma_{D}[1,0,0,0,0, \mathbf{0}], \quad \boldsymbol{b}_{G}=\sigma_{G}[0,1,0,0,0, \mathbf{0}], \\
& \boldsymbol{b}_{q}=\sigma_{q}\left[\kappa_{D q}, 0,0,0, \sqrt{1-\kappa_{D q}^{2}}, \mathbf{0}\right] \quad \boldsymbol{b}_{1}=\sigma_{1}\left[0,0, \kappa_{-}, \kappa_{+}, 0, \mathbf{0}\right], \\
& \boldsymbol{b}_{2}=\sigma_{2}\left[0,0, \kappa_{+}, \kappa_{-}, 0, \mathbf{0}\right], \quad \boldsymbol{b}_{U}=\sigma_{U}[0,0,0,0,0, \mathbf{1}],
\end{aligned}
$$

where $\kappa_{D q} \in(-1,1)$ and $\kappa_{ \pm}=\frac{1}{2}\left(\sqrt{1+\kappa_{12}} \pm \sqrt{1-\kappa_{12}}\right)$. This specification of the underlying shocks has simple interpretations. For example, $w_{D, t}$ fully characterizes instantaneous shocks to the dividend $D_{t}$ and $\sigma_{D}$ gives its instantaneous volatility. This form of the $\boldsymbol{b}$ 's gives a particular correlation structure among the shocks to different variables. In particular, $\kappa_{D q}$ is the correlation between the payoffs from the stock and the investors' private technologies. We choose this correlation structure to simplify exposition, and our results are not sensitive to this particular choice.

\section{F. Additional Notation}

We also introduce some notation. Let $\mathrm{E}_{i, t}=\mathrm{E}\left[\cdot \mid \mathcal{I}_{i, t}\right](i=1,2)$ and $\mathrm{E}_{t}=$ $\mathrm{E}\left[\cdot \mid \mathcal{I}_{t}\right]$. Define

$$
F_{t}=\mathrm{E}_{t}\left[\int_{t}^{\infty} e^{-r(s-t)} d D_{s}\right]=\frac{1}{r+a_{G}} G_{t}
$$

${ }^{13}$ Intraday trading and return patterns of stocks cross-listed on exchanges with different trading hours provide interesting evidence on this point. See, for example, Chan et al. (1996) and Kleidon and Werner (1996). 
$F_{t}$ gives the expected value of future dividends on the stock discounted at the risk-free rate under full information and $d F_{t}=-\left[a_{G} /\left(r+a_{G}\right)\right] G_{t} d t+$ $\boldsymbol{b}_{F} d \boldsymbol{w}_{t}$ where $\boldsymbol{b}_{F}=\left[1 /\left(r+a_{G}\right)\right] \boldsymbol{b}_{G}$. Also, define

$$
d Q_{t}=d P_{t}+d D_{t}-r P_{t} d t \quad \forall t \in \mathcal{T}
$$

to be the instantaneous excess return on one stock share when the market is open and $e=\mathrm{E}\left[d Q_{t}\right] / d t, \sigma_{Q}^{2}=\mathrm{E}\left[\left(d Q_{t}\right)^{2}\right] / d t$ its first two unconditional moments. In order to relate to the empirical findings, we also define the simple share return on the stock from $s$ to $t$ to be

$$
R_{s, t}=P_{t}-P_{s}+\int_{s}^{t} D_{\tau} d \tau
$$

where $s, t \in \mathcal{T}$ and $t>s$.

\section{Definition of Equilibrium}

In order to derive an equilibrium for the economy, we begin by stating each investor's budget constraint and optimization problem under a given stock price process $P_{t}$. Investor $i$ 's financial wealth at time $t$ consists of two parts: the balance in a money-market account denoted by $W_{i, t}^{0}$ and the position in stock denoted by $\theta_{i, t}$. During the day, that is, $t \in \mathcal{T}_{k}(k=0,1, \ldots)$, the stock market is open and his stock position can be marked to the market. Investor $i$ 's total financial wealth is then given by the market value of his stock position and moneymarket account: $W_{i, t}=W_{i, t}^{0}+\theta_{i, t} P_{t}$. He can freely adjust his stock and moneymarket positions to finance consumption and private investments. His budget constraint is specified by the dynamics of his total financial wealth $W_{i, t}$. During the night (i.e., $t \in \mathcal{N}_{k}$ ), the stock market is closed and investor $i$ holds his position from the previous day, $\theta_{i, n_{k}}$, which can no longer be marked to the market. Investor $i$ now has to finance his consumption and private investments through his money-market account. His budget constraint is specified by the dynamics of his money-market account $W_{i, t}^{0}$. His overnight stock position $\theta_{i, n_{k}}$ now acts as an additional state variable.

Each investors' optimization problem can now be expressed as follows. For $i=1,2$ and $k=0,1, \ldots$,

$$
\begin{aligned}
t \in \mathcal{T}_{k}: \quad J_{i, t}= & \sup _{\left\{c_{i}, y_{i}, \theta_{i}\right\}} \mathrm{E}\left[-\int_{t}^{n_{k}} e^{-\rho(s-t)-\gamma c_{i, s}} d s+J_{i, n_{k}^{+}}^{*} \mid \mathcal{I}_{i, t}\right] \\
& \text { s.t. } \quad d W_{i, t}=\left(r W_{i, t}-c_{i, t}\right) d t+\theta_{i, t} d Q_{t}+y_{i, t} d q_{i, t} \\
t \in \mathcal{N}_{k}: \quad J_{i, t}^{*}= & \sup _{\left\{c_{i}, y_{i}\right\}} \mathrm{E}\left[-\int_{t}^{t_{k+1}} e^{-\rho(s-t)-\gamma c_{i, s}} d s+J_{i, t_{k+1}} \mid \mathcal{I}_{i, t}\right] \\
& \text { s.t. } d W_{i, t}^{0}=\left(r W_{i, t}^{0}-c_{i, t}\right) d t+\theta_{i, n_{k}} d D_{t}+y_{i, t} d q_{i, t},
\end{aligned}
$$


where $J_{i, t}, J_{i, t}^{*}$ denote, respectively, investor $i$ 's value function during the day and night, and $t^{-}, t^{+}$, respectively, the time right before and after $t$. At the close and the open, the optimality of investors' policies yields the following boundary conditions:

$$
J_{i, n_{k}}=\mathrm{E}\left[J_{i, n_{k}^{+}}^{*} \mid \mathcal{I}_{i, n_{k}}\right] \text { and } J_{i, t_{\bar{k}+1}^{-}}^{*}=\mathrm{E}\left[J_{i, t_{k+1}} \mid \mathcal{I}_{i, t_{\bar{k}+1}}\right]
$$

An equilibrium is given by a stock price process $\left\{P_{t}, t \in \mathcal{T}\right\}$ such that investors follow their optimal policies and the stock market clears. The market clearing condition is

$$
\omega \theta_{1, t}+(1-\omega) \theta_{2, t}=1 \quad \forall t \in \mathcal{T} .
$$

The equilibrium stock price and the investors' optimal policies can in general be expressed as functions of time and the state of the economy. The state of the economy is determined by investors' wealth and their information on current and future investment opportunities. We make two assumptions about the investors' information sets, which are verified later: (a) $\mathcal{I}_{i, t}$ has a finite dimensional sufficient statistic $\boldsymbol{\Psi}_{i, t}$, and (b) $\boldsymbol{\Psi}_{t}=\left\{\boldsymbol{\Psi}_{1, t}, \boldsymbol{\Psi}_{2, t}\right\}$ follows a Markov process under $\mathcal{I}_{i, t}(i=1,2)$. Under these two assumptions, $\Psi_{t}$ together with investors' wealth defines a set of variables that fully characterize the state of the economy at time $t$. We can then write $P_{t}=P(\bullet ; t)$ (for $t \in \mathcal{T})$ and $\left\{c_{i, t}, y_{i, t}, \theta_{i, t}\right\}=\left\{c_{i}(\bullet ; t), y_{i}(\bullet ; t), \theta_{i}(\bullet ; t)\right\}($ for $i=1,2)$, where $\bullet$ denotes the relevant state variables. (Given that each investor's policy is adapted to his information, it may only depend on a subset of the state variables.) We call $P(\bullet ; t)$ the price function and $\left\{c_{i}(\bullet ; t), y_{i}(\bullet ; t), \theta_{i}(\bullet ; t)\right\}$ the policy function of investor $i$.

The price function and policy functions are time-dependent since the structure of the economy changes over time. For example, the daytime and the nighttime are different because the stock market opens only during the day. Different points in time during a day are also different because the trading opportunities remaining in the day are different. Given that $N$ and $T$ are assumed to be the same for all $k$, the time variation is periodic: the day and the night repeat themselves over time. Thus, we only consider periodic equilibria of the economy, in which the price function and investors' policy functions exhibit periodicity in time. Specifically, we have

Definition 1: A periodic equilibrium is defined by the stock price function $P(\bullet ; t)$ and investors' policy functions $\left\{c_{i}(\bullet ; t), y_{i}(\bullet ; t), \theta_{i}(\bullet ; t)\right\}, i=1,2$, such that (a) the policies maximize investors' expected utility, (b) the stock market clears, and (c) the price function and the investors' policy functions are periodic in $t$ with periodicity $T+N$.

Under Definition 1, the stock price depends on the relevant state variables in the same way for every day. That is,

$$
P(\bullet ; \tau)=P\left(\bullet ; t_{k}+\tau\right),
$$


where $k=0,1, \ldots$ and $\tau \in[0, T]$. Realized values of the stock price can be different from day to day as values of the state variables can be different. Furthermore, the periodicity in each investor's optimization problem and policy function yields

$$
J_{i}\left(\bullet ; t_{k}\right)=J_{i}\left(\bullet ; t_{k+1}\right) \quad(i=1,2) ;
$$

that is, his value function, as a function of the state variables, is periodic in time with periodicity $T+N$. Equations (12) and (13) provide the boundary conditions we need to solve a periodic equilibrium. Thus, a periodic equilibrium of the economy is given by a price function (12) such that investors adopt optimal policies given by the solution to equations (9), (10), and (13), and the market clears as equation (11) requires.

Given the constant risk-free rate and constant absolute risk aversion in preferences, each investor's demand of risky investments depends only on the distribution of future payoffs, not on his wealth (see, e.g., Merton (1969)). We thus seek an equilibrium in which the stock price is independent of investors' wealth; that is, $P_{t}=P\left(\Psi_{t} ; t\right)$. Furthermore, in this paper we restrict ourselves to the linear equilibria in which the price function is linear in $\Psi_{t}$.

Definition 2: A linear, periodic equilibrium is a periodic equilibrium in which $P_{t}=L\left(\Psi_{t} ; t\right) \forall t \in \mathcal{T}$, where $L(\cdot ; t)$ denotes a linear function, which is time dependent with periodicity $T+N$, and $\Psi_{t}$ denotes the set of sufficient statistics for $\mathcal{I}_{1, t}$ and $\mathcal{I}_{2, t}$.

\section{Solution of the Equilibrium}

In solving for an equilibrium, we first conjecture a particular equilibrium and then we characterize the investors' optimal policies and market clearing conditions under that conjectured equilibrium. Finally, we confirm that the conjectured equilibrium indeed exists.

\section{A. Conjecture of the Equilibrium}

In the current model, the distribution of future payoffs on the stock and private technologies is fully determined by $\boldsymbol{Z}_{t}=\left[G_{t}, Y_{1, t}, Y_{2, t}\right]^{\prime}$. In general, investors do not have full information on $\boldsymbol{Z}_{t}$ and need to rely on their own information in decision making. In seeking a linear, periodic equilibrium, we conjecture a price function linear in the conditional expectations of both investors:

$$
P_{t}=\lambda_{1} \mathrm{E}_{1, t}\left[\boldsymbol{Z}_{t}\right]+\lambda_{2} \mathrm{E}_{2, t}\left[\boldsymbol{Z}_{t}\right]-\lambda_{0} \quad \forall t \in \mathcal{T},
$$

where $\lambda_{i}=\left[\lambda_{i G},-\lambda_{i 1},-\lambda_{i 2}\right](i=1,2)$, defined on $\mathcal{T}$, is deterministic but time-dependent with periodicity $T+N$. Let $\hat{G}_{t}=\mathrm{E}_{2, t}\left[G_{t}\right]$ and $\hat{Y}_{1, t}=\mathrm{E}_{2, t}\left[Y_{1, t}\right]$. From equation (14), it follows that

$$
P_{t}=\lambda_{1 G} G_{t}-\lambda_{11} Y_{1, t}-\lambda_{12} Y_{2, t}+\lambda_{2 G} \hat{G}_{t}-\lambda_{21} \hat{Y}_{1, t}+\lambda_{22} Y_{2, t}-\lambda_{0} .
$$




\section{B. Characterization of the Equilibrium}

To characterize the equilibrium, we take the price function, equation (15), as given and derive each investor's expectations and policies and conditions for market clearing.

\section{B.1. Conditional Expectations}

Investor $i$ receives a vector of signals $\boldsymbol{S}_{i, t}: \boldsymbol{S}_{1, t}=\left[\boldsymbol{U}_{t} ; D_{t} ; P_{t} ; G_{t} ; Y_{1, t} ; Y_{2, t} ; q_{1, t}\right]$ and $\boldsymbol{S}_{2, t}=\left[\boldsymbol{U}_{t} ; D_{t} ; P_{t} ; Y_{2, t} ; q_{2, t}\right]$. Thus, $\mathcal{I}_{i, t}=\left\{\boldsymbol{S}_{i, t}: 0 \leq s \leq t\right\}$. Since $\boldsymbol{Z}_{t}$ and $\boldsymbol{S}_{i, t}$ are jointly Gaussian, the distribution of $\boldsymbol{Z}_{t}$ conditioned on $\left\{\boldsymbol{S}_{i, t}: 0 \leq s \leq t\right\}$ is Gaussian, fully characterized by its mean and variance. Let $\boldsymbol{Z}_{i, t}=\mathbf{E}_{i, t}\left[\boldsymbol{Z}_{t}\right]$ and $\boldsymbol{o}_{i}(t)=\mathrm{E}_{i, t}\left[\left(\boldsymbol{Z}_{t}-\boldsymbol{Z}_{i, t}\right)\left(\boldsymbol{Z}_{t}-\boldsymbol{Z}_{i, t}\right)^{\prime}\right]$ denote investor $i$ 's conditional mean and variance of $\boldsymbol{Z}_{t}$, respectively. We have the following lemma.

Lemma 1: Given equation (3), $\boldsymbol{S}_{i, t}$, and equation (15), $\boldsymbol{Z}_{t}$ is a Gaussian process under $\mathcal{I}_{i, t}, i=1,2$. That is, $\forall s, s_{1}, \ldots, s_{n}(\geq 0),\left\{\boldsymbol{Z}_{s_{1}}, \ldots, \boldsymbol{Z}_{s_{n}}\right\}$ has a Gaussian distribution conditioned on $\mathcal{I}_{i, s}$. Furthermore, $\boldsymbol{Z}_{i, t}$ and $\boldsymbol{o}_{i}(t)$ are governed by

$$
\begin{aligned}
\forall t>s: \quad \boldsymbol{Z}_{i, t} & =e^{-\boldsymbol{a}_{Z}(t-s)} \boldsymbol{Z}_{i, s}+\int_{s}^{t} e^{-\boldsymbol{a}_{Z}(t-\tau)} \boldsymbol{k}_{i}\left(d \boldsymbol{S}_{i, \tau}-\mathrm{E}_{i, \tau}\left[d \boldsymbol{S}_{i, \tau}\right]\right) ; \\
\boldsymbol{o}_{i}(t) & =\boldsymbol{o}_{i}(s)+\int_{s}^{t} \boldsymbol{g}_{i, o} d \tau,
\end{aligned}
$$

where $\boldsymbol{k}_{i}$ and $\boldsymbol{g}_{i, o}$ are deterministic, given in Appendix B. In particular, $\boldsymbol{o}_{i}$ evolves deterministically over time.

Given the solution to $\boldsymbol{Z}_{i, t}$ in Lemma 1, the next result is immediate:

LemMa 2: Let $\boldsymbol{\Psi}_{t}=\left[\boldsymbol{Z}_{1, t} ; \boldsymbol{Z}_{2, t}\right]$ and $\boldsymbol{\Psi}_{i, t}=\mathbf{E}_{i, t}\left[\boldsymbol{\Psi}_{t}\right], i=1,2 . \boldsymbol{\Psi}_{i, t}$ is a sufficient statistic for $\mathcal{I}_{i, t}$, following a Gaussian Markov process under $\mathcal{I}_{i, t}$, and $\Psi_{t}$ is a set of sufficient statistics for $\mathcal{I}_{1, t}$ and $\mathcal{I}_{2, t}$, following a Gaussian Markov process under $\mathcal{I}_{t}$ as well as under $\mathcal{I}_{i, t}$.

Lemma 2 verifies the two assumptions in Section II about the investors' information sets in a linear equilibrium. We can define $\boldsymbol{\Psi}_{t}=\left[\boldsymbol{Z}_{1, t} ; \boldsymbol{Z}_{2, t}\right]$ to be the state vector.

Lemmas 1 and 2 apply under the conjectured linear price function, equation (14), even when the lengths of the day and night change through time. For our purpose, the lengths of the day and night are assumed to be constant and we seek a periodic solution to the investors' expectations, which requires that

$$
\boldsymbol{o}_{i}\left(t_{k+1}\right)=\boldsymbol{o}_{i}\left(t_{k}\right), \quad k=0,1,2, \ldots
$$




\section{B.2. Optimal Policies}

For future convenience, we define $\boldsymbol{X}_{t}=\left[1, Y_{1, t}, Y_{2, t}, G_{t}-\hat{G}_{t}, Y_{1, t}-\hat{Y}_{1, t}\right]^{\prime}$ as a subvector of the state variables and let $\boldsymbol{X}_{i, t}=\mathrm{E}_{i, t}\left[\boldsymbol{X}_{t}\right]$ denote investor $i$ 's conditional expectation of $\boldsymbol{X}_{t}$. The price function in equation (15) can then be rewritten as follows:

$$
P_{t}=F_{t}-\lambda \boldsymbol{X}_{t}=F_{i, t}-\lambda_{i} \boldsymbol{X}_{i, t} \quad \forall t \in \mathcal{T},
$$

where $\lambda=\left[\lambda_{0}, \lambda_{1}, \lambda_{2}, 1 /\left(r+a_{G}\right)-\lambda_{G}, 0\right], F_{i, t}=\mathrm{E}_{i, t}\left[F_{t}\right], \lambda_{1}=\lambda$, and $\lambda_{2}=$ $\left[\lambda_{0}, \lambda_{1}, \lambda_{2}, 0,0\right] .{ }^{14}$ From Lemma $1, \boldsymbol{X}_{i, t}$ follows a Gaussian Markov process under $\mathcal{I}_{i, t}$ and we have

$$
\forall t>s: \quad \boldsymbol{X}_{i, t}=\boldsymbol{X}_{i, 0}+\int_{0}^{t}\left\{-\boldsymbol{a}_{i, X} \boldsymbol{X}_{i, s} d s+\boldsymbol{k}_{i, X}\left(d \boldsymbol{S}_{i, s}-\mathrm{E}_{i, t}\left[d \boldsymbol{S}_{i, s}\right]\right)\right\}
$$

and

$$
\forall t \in \mathcal{T}: \quad Q_{t}=\int_{0}^{t}\left\{\boldsymbol{a}_{i, Q} \boldsymbol{X}_{i, t} d t+\boldsymbol{k}_{i, Q}\left(d \boldsymbol{S}_{i, t}-\mathrm{E}_{i, t}\left[d \boldsymbol{S}_{i, t}\right]\right)\right\},
$$

where $i=1,2, \boldsymbol{a}_{i, X}, \boldsymbol{k}_{i, X}, \boldsymbol{a}_{i, Q}, \boldsymbol{b}_{i, Q}$ are given in Appendix B.

With equations (19) and (20), investor $i$ 's control problem as defined in equation (9) can be solved explicitly. The following lemma summarizes the result:

Lemma 3: Given the price function of equation (18) and the solution to investors' expectations of equations (16) and (17), investor i's value function has the form

$$
\begin{cases}t \in \mathcal{T}_{k}: & J_{i, t}=-\exp \left\{-\rho t-r \gamma W_{i, t}-\frac{1}{2} \boldsymbol{X}_{i, t}^{\prime} \boldsymbol{v}_{i} \boldsymbol{X}_{i, t}\right\} \\ t \in \mathcal{N}_{k}: & J_{i, t}^{*}=-\exp \left\{-\rho t-r \gamma W_{i, t}^{*}-\frac{1}{2} \boldsymbol{X}_{i, t}^{* \prime} \boldsymbol{v}_{i} \boldsymbol{X}_{i, t}^{*}\right\}\end{cases}
$$

where $i=1,2, k=0,1, \ldots, W_{i, t}^{*}=W_{i, t}^{0}+\theta_{i, n_{k}} F_{i, t}, \boldsymbol{X}_{i, t}^{*}=\left[\boldsymbol{X}_{i, t} ; \theta_{i, n_{k}}\right], \boldsymbol{v}_{i}$ is a symmetric matrix, given by

$$
\forall s<t: \quad \boldsymbol{v}_{i}(s)=\boldsymbol{v}_{i}(t)+\int_{t}^{s} \boldsymbol{g}_{i, v} d \tau
$$

${ }^{14}$ Equation (15) can be rewritten as $P_{t}=\left(\lambda_{1 G}+\lambda_{2 G}\right) G_{t}-\lambda_{0}-\left(\lambda_{11}+\lambda_{21}\right) Y_{1, t}-\left(\lambda_{12}+\right.$ $\left.\lambda_{22}\right) Y_{2, t}-\lambda_{2 G}\left(G_{t}-\hat{G}_{t}\right)+\lambda_{21}\left(Y_{1, t}-\hat{Y}_{1, t}\right)$. Since $\lambda_{1 G}\left(G_{t}-\hat{G}_{t}\right)=\lambda_{11}\left(Y_{1, t}-\hat{Y}_{1, t}\right)$ for $t \in \mathcal{T}$, there is no loss of generality in eliminating $\left(Y_{1, t}-\hat{Y}_{1, t}\right)$ from the price function (by redefining $\left.\lambda_{2 G}\right)$. No arbitrage requires that $\lambda_{1 G}+\lambda_{2 G}=1 /\left(r+a_{G}\right)$. We then obtain the form in equation (18) with $\lambda_{1}=\lambda_{11}+\lambda_{21}, \lambda_{2}=\lambda_{12}+\lambda_{22}$, and $\lambda_{G}=\lambda_{1 G}$. 
and

$$
\boldsymbol{v}_{i}\left(t_{k}\right)=\boldsymbol{v}_{i}\left(t_{k+1}\right)
$$

with $\boldsymbol{g}_{i, v}$ being deterministic, given in Appendix B. His optimal policies are

$$
\begin{aligned}
& t \in\left[t_{k}, n_{k}\right]:\left\{\begin{array}{l}
{\left[\begin{array}{l}
\theta_{i, t} \\
y_{i, t}
\end{array}\right]=\left[\begin{array}{l}
\boldsymbol{h}_{i, \theta} \\
\boldsymbol{h}_{i, y}
\end{array}\right] \boldsymbol{X}_{i, t}} \\
c_{i, t}=-\frac{1}{r} \ln \gamma+r W_{i, t}+\frac{1}{2 \gamma} \boldsymbol{X}_{i, t}^{\prime} \boldsymbol{v}_{i} \boldsymbol{X}_{i, t}
\end{array}\right. \\
& t \in\left(n_{k}, t_{k+1}\right):\left\{\begin{array}{l}
y_{i, t}=\left[\boldsymbol{h}_{i, y}, h_{i}\right] \boldsymbol{X}_{i, t}^{*} \\
c_{i, t}=-\frac{1}{r} \ln \gamma+r W_{i, t}^{*}+\frac{1}{2 \gamma} \boldsymbol{X}_{i, t}^{* \prime} \boldsymbol{v}_{i} \boldsymbol{X}_{i, t}^{*},
\end{array}\right.
\end{aligned}
$$

where $\boldsymbol{h}_{i, \theta}, \boldsymbol{h}_{i, y}, h_{i}$ are functions of $\boldsymbol{\lambda}_{i}, \boldsymbol{o}_{i}, \boldsymbol{v}_{i}$, given in Appendix $B$.

Investors' optimal investment policies, $\theta_{i, t}$ and $y_{i, t}(i=1,2)$, are both linear functions of $\boldsymbol{X}_{i, t}$. During the day, the stock market is open; investors can freely trade the stock and adjust their private investments. During the night, the stock market is closed and investors hold their closing stock positions from the previous day, which is $\theta_{i, n_{k}}$ for $t \in\left(n_{k}, t_{k+1}\right)$. Investor $i$ 's investment in his private technology and consumption not only depend on $\boldsymbol{X}_{i, t}$, but also depend on $\theta_{i, n_{k}}$.

Given $\boldsymbol{\lambda}$ and the resulting $\boldsymbol{o}_{i}$, Lemma 3 expresses investor $i$ 's optimal policies as functions of $\boldsymbol{v}_{i}$, which is solved from equation (22), a (vector-form) first-order ordinary differential equation (ODE), with boundary condition (23).

\section{B.3. Market Clearing}

In equilibrium, the stock market clears. From Lemma 3, the market clearing condition requires

$$
\omega \lambda_{1} \boldsymbol{h}_{1, \theta}+(1-\omega) \boldsymbol{h}_{2, \theta} \boldsymbol{\tau}=[1,0,0,0,0] \quad \forall t \in \mathcal{T}_{k} \quad(k=0,1, \ldots),
$$

where $\tau=\left[\left[\lambda_{1}, 0,0,0,0\right] ;\left[0, \lambda_{1}, 0,-\lambda_{G}, 0\right] ;\left[0,0, \lambda_{1}, 0,0\right]\right]$. The periodic condition further requires that, for $k=0,1,2, \ldots$,

$$
\lambda\left(t_{k}\right)=\lambda\left(t_{k+1}\right)
$$


Given $\boldsymbol{h}_{i, \theta}(i=1,2)$, equation (25) defines a first-order ODE for $\boldsymbol{\lambda}$ (see Appendix $C$ ) and equation (26) defines the boundary condition.

\section{Existence and Computation of the Equilibrium}

Lemmas 1, 2, and 3 specify the investors' expectations and optimal policies and the stock market clearing condition in a linear, periodic equilibrium of the form in equation (14) or equivalently equation (15). Solving such an equilibrium now reduces to solving equations (16b), (22), and (25), a system of first-order ODEs for $\boldsymbol{o}_{1}, \boldsymbol{o}_{2}, \boldsymbol{v}_{1}, \boldsymbol{v}_{2}$, and $\lambda$, subject to periodicity conditions (17), (23), and (26).

The solution of an ODE system depends on the boundary condition. In the case of the familiar initial-value problem, the boundary condition is simply the initial value of the system. It seeks a solution given its value at a fixed point in time. Our problem, however, has a different boundary condition. We need to find particular initial values $\boldsymbol{o}_{i}\left(t_{k}\right), \boldsymbol{v}_{i}\left(t_{k}\right)(i=1,2)$, and $\boldsymbol{\lambda}\left(t_{k}\right)$ such that equations (17), (23), and (26) hold. This is known as a two-point boundary value problem, which seeks a solution of the system with its values at two given points in time satisfying a particular condition.

The following theorem states the result on the existence of a solution to the given system, which gives a linear, periodic equilibrium of the economy.

Theorem 1: For $\omega$ close to one, a linear, periodic equilibrium exists (generically), in which the stock price has the form in equation (15), the investors' expectations are given by equations (16a) and (16b), and the investors' policies are given by equation (24). 15

The proof of Theorem 1 is given in Appendix C.

In general, the actual values of $\boldsymbol{o}_{i}, \boldsymbol{v}_{i}(i=1,2)$, and $\boldsymbol{\lambda}$, can only be solved numerically. In solving the system, we always start with the solution at $\omega=$ 1 and then obtain solutions for values of $\omega$ close to one. Iteratively, we arrive at the solutions for desired values of $\omega$. (The method for numerical solutions is discussed in more detail in Appendix E.)

\section{The Case of Symmetric Information}

We first analyze the equilibrium in the case of symmetric information when $\mathcal{I}_{i, t}=\mathcal{I}_{t} \forall t \geq 0$ and $i=1,2$; all investors have perfect information about the economy and trade only for hedging reasons. This case can be obtained from the general model by letting $\boldsymbol{U}_{0}=\boldsymbol{Z}_{0}, \boldsymbol{a}_{U}=[[1,0,0] ;[0,1,0] ;[0,0,1]]$, and $\boldsymbol{b}_{U}=0$.

${ }^{15}$ We assume that the investor's initial information given by $\boldsymbol{U}_{0}$ is consistent with what the periodic equilibrium requires. Here, the condition that $\omega$ is close to one arises from the specific approach we use in the proof as opposed to economic reasons (see Appendix C). Our proof relies on a continuity argument. It is first shown that at $\omega=1$, a solution to the given system exists. Since the system is smooth with respect to $\omega$, it is then shown that a solution also exists for $\omega$ close to one. The proof itself, however, does not specify the required range of $\omega$. 
$G_{t}, Y_{1, t}$, and $Y_{2, t}$ then become public information. Studying the case of symmetric information allows us to examine how closures affect investors' hedging trade and the resulting trading and return patterns.

\section{A. Equilibrium under Symmetric Information}

From the general solution of the equilibrium in Section III, the stock price under symmetric information is

$$
P_{t}=F_{t}-\left(\lambda_{0}+\lambda_{1} Y_{1, t}+\lambda_{2} Y_{2, t}\right) \quad \forall t \in \mathcal{T} .
$$

It is given by the value of expected future dividends $\left(F_{t}\right)$ minus a risk discount $\left(\lambda_{0}+\sum_{i} \lambda_{i} Y_{i, t}\right)$. The risk discount consists of two parts: (a) an unconditional part $\left(\lambda_{0}\right)$ that is independent of the returns on the alternative private investments and (b) a conditional part that depends on the expected returns on the private investments. The unconditional part derives primarily from the uncertainty in future dividends. ${ }^{16}$ The conditional part of the risk discount depends on the correlation between the returns on the stock and the private technologies, $\kappa_{D q}$. When the expected return on investor $i$ 's private technology is high, he wants to increase his investment in private technology. If the return on private technology is uncorrelated with the return on the stock $\left(\kappa_{D q}=0\right)$, he can finance the additional private investment solely via borrowing at the risk-free rate and his holdings of the stock do not change. If there is positive correlation $\left(\kappa_{D q}>0\right)$, however, some of the stock is sold to control the risk of his overall portfolio of risky assets, or if there is negative correlation $\left(\kappa_{D q}<0\right)$, more stock is purchased (financed by borrowing). Thus, except when $\kappa_{D q}=0$, holding the stock price fixed, changes in the expected return on private technologies affect the optimal holdings of the stock. In equilibrium, the price of the stock must change in order to induce other investors to hold amounts of the stock that clear the market. When $\kappa_{D q}>0(<0)$, the stock price is lower (higher) for higher expected returns on private technologies; when $\kappa_{D q}=0$, stock prices are unaffected by the expected returns on private technologies.

${ }^{16}$ From equation (1), the stock's future dividends can be decomposed into two components: a riskless component determined by current $G_{t}$ and a risky component independent of $G_{t}$ with zero mean. In particular, $\forall t \geq s \geq 0, G_{t}=e^{-a_{G}(t-s)} G_{s}+\int_{s}^{t} e^{-a_{G}(\tau-s)} b_{G} d w_{\tau}$ and

$$
\begin{aligned}
D_{t}-D_{s} & =\int_{s}^{t} G_{\tau} d \tau+\int_{s}^{t} b_{D} d w_{\tau} \\
& =\left[\int_{s}^{t} e^{-a_{G}(\tau-s)} d \tau\right] G_{s}+\left[\int_{s}^{t} d \tau \int_{s}^{\tau} e^{-a_{G}\left(\tau^{\prime}-s\right)} b_{G} d w_{\tau^{\prime}}+\int_{s}^{t} b_{D} d w_{\tau}\right] .
\end{aligned}
$$

The first term gives the deterministic component in future dividends and the second term gives the risky component, which has zero mean conditioned on the information at $s$. The present value of the riskless component is $F_{t}$. The present value of the risky component is $-\lambda_{0}$. In other words, $\lambda_{0}$ gives the risk premium associated with the risky component of dividends. 
Investor $i$ 's stock position during the day is given by

$$
\theta_{i, t}=h_{i, \theta}^{(0)}+h_{i, \theta}^{(1)} Y_{1, t}+h_{i, \theta}^{(2)} Y_{2, t} \quad(i=1,2) .
$$

As an example, we consider investor 1's stock position, $\theta_{1, t}$, which consists of three components. The first component $h_{1, \theta}^{(0)}$ gives his unconditional stock position. The second component $h_{1, \theta}^{(1)} Y_{1, t}$ arises from his hedging trade as he adjusts his stock position to hedge the risk of his private investments. The third component $h_{1, \theta}^{(2)} Y_{2, t}$ arises from his market-making activity. As class 2 investors trade the stock to hedge the risk of their private investments, the price adjusts to induce class 1 investors to take the opposite position, leading to the third component in his stock position (which is proportional to $\left.Y_{2, t}\right)$. So, $h_{1, \theta}^{(1)}$ and $h_{1, \theta}^{(2)}$ characterize the intensity of investor 1's hedging and market-making activities in equilibrium, respectively. ${ }^{17}$

\section{B. Time Variation in Trading and Returns}

We now examine how market closures give rise to time variation in investors' hedging trade and the resulting stock price. The intensity of investors' hedging trade depends on its cost and benefit. In particular, the lack of overnight trading opportunities increases the risk of overnight stock positions. The increase in hedging cost causes investors to decrease their overnight hedging positions at the close. In a continuous-time setting like ours, the low hedging position desired at the close need not affect investors' hedging trade during the day. They can hedge actively throughout the day and reduce their hedging position discretely at the close, giving a discontinuity in $\boldsymbol{h}_{i, \theta}=\left[h_{i, \theta}^{(0)}, h_{i, \theta}^{(1)}, h_{i, \theta}^{(2)}\right]$ at $n_{k}(k=0,1, \ldots)$.

The low hedging demand at the close makes the market clearing price at the close to be less sensitive to private technological shocks and $\lambda_{i}(i=1,2)$ is lower than it would be if the market remained open. Moreover, the price at the close also affects the prices prior to the close. Unlike investors' hedging position, which goes through discrete changes at the close, the market clearing price cannot change discretely. In our continuous-time setting, the information flow is continuous (the state variables follow continuous paths). Any discrete price change would be predictable and lead to arbitrage oppor-

\footnotetext{
17 The above interpretation of an investor's stock holding is an oversimplification for ease of exposition. The economic forces behind each of the components (defined as part of the holding associated with a particular state variable) are more complicated. Take investor 1's hedging component as an example. It originates from his hedging needs in response to technology shock $Y_{1, t}$. As his cohort trades in the same direction, the expected return on the stock changes, which in turn affects his stock holding. Moreover, the stock return now becomes correlated with changes in expected returns on private investments and the stock itself, and investor 1 also uses the stock to hedge against these changes. The net dependence of his stock holding on $Y_{1, t}$ has contributions from all these channels. See Merton $(1971,1990)$ for general discussions of investors' optimal asset demands when expected returns are stochastic.
} 
tunities. ${ }^{18}$ Hence, $\lambda_{i}$ being lower at the close also makes it lower before the close. Of course, the effect of the close diminishes as we move further away from the close. In short, closures cause discrete changes in investors' hedging trade at the close, which generate smooth changes in the price over the day.

\section{B.1. Special Case 1: Homogeneous Investors}

In order to develop more intuition about the model, we first consider a special case in which investors are homogeneous. We establish the simple result that in the absence of any need to trade among investors, closures generate no time variation in prices.

Let $\sigma_{1}=\sigma_{2}, \kappa_{12}=1$, and $Y_{1,0}=Y_{2,0}$. Thus, $Y_{1, t}=Y_{2, t} \forall t \geq 0$, which we denote by the common process $Y_{t}$ with volatility $\sigma_{Y}$. The two classes of investors then face the same investment opportunities, both public and private. In this case, all investors become identical, the market is effectively complete, and the equilibrium is simple.

Proposition 1: When $\sigma_{1}=\sigma_{2}=\sigma_{Y}$ and $\kappa_{12}=1$, the economy has a unique linear, periodic equilibrium. The equilibrium stock price is

$$
P_{t}=F_{t}-\left(\bar{\lambda}_{0}+\bar{\lambda} Y_{t}\right) \quad \forall t \in \mathcal{T},
$$

where

$$
\bar{\lambda}_{0}=\gamma\left(\bar{\sigma}_{D}^{2}+\sigma_{F}^{2}+\bar{\lambda}^{2} \sigma_{Y}^{2}\right) \equiv \bar{\lambda}_{00}+\gamma \bar{\lambda}^{2} \sigma_{Y}^{2} \quad \text { and } \quad \bar{\lambda}=\frac{\sigma_{D} \kappa_{D q}}{\sigma_{q}\left(r+a_{Y}+\sigma_{Y}^{2} v\right)}
$$

with $\bar{\sigma}_{D}^{2}=\sigma_{D}^{2}\left(1-\kappa_{D q}^{2}\right)$ and $v=\left(1 / 2 \sigma_{Y}^{2}\right)\left[-\left(r+2 a_{Y}\right)+\sqrt{\left(r+2 a_{Y}\right)^{2}+4 \sigma_{Y}^{2} / \sigma_{q}^{2}}\right]$.

Proofs of the propositions are given in Appendix D.

Clearly, $\bar{\lambda}_{0} \geq 0$ and $\bar{\lambda} \geq 0$ (assuming $\kappa_{D q} \geq 0$ ). The equilibrium price function is now independent of $T$ and $N$. Being identical, all investors hold equal shares of the market (day or night) and there is no trading. How the market closes and opens has no effect on investors' investment-consumption decisions and the stock price function.

Certain properties of the equilibrium are useful in future discussions, especially how it depends on the uncertainty in private investment opportunities. First, $\sigma_{Y}$ gives the volatility of investors' private investment opportunities. With correlated payoffs, more volatile private investments make

\footnotetext{
${ }^{18}$ See, for example, Huang (1985) for a discussion of general conditions under which asset prices must follow diffusion processes. Although Huang's results are derived for the case of complete markets, extending them to the case of incomplete markets as ours is intuitively straightforward.
} 
the stock riskier, leading to a higher (unconditional) risk discount on the stock. In particular, for $\sigma_{Y}=0$ (constant private investment opportunities), $\bar{\lambda}_{00}=\gamma\left(\bar{\sigma}_{D}^{2}+\sigma_{F}^{2}\right)$ gives the risk discount, which is proportional to the aggregate risk-aversion and the total risk of the stock. ${ }^{19}$ For $\sigma_{Y}>0, \bar{\lambda}_{0}$ is greater than $\bar{\lambda}_{00}$. The difference, $\gamma \bar{\lambda}^{2} \sigma_{Y}^{2}$, gives the extra premium associated with stochastic private investments, which increases with $\sigma_{Y}^{2}$. Moreover, as the stock becomes riskier, investors reduce the level of their private investments (for a given expected return). As a result, shocks to their private investment opportunities have less impact on the stock price, leading to a smaller $\bar{\lambda}$. Additionally, $a_{Y}$ determines the persistence in private technological shocks. For more transitory shocks (larger $a_{Y}$ ), they have less effect on the risk of the stock. Thus, both $\bar{\lambda}_{0}$ and $\bar{\lambda}$ become smaller for larger $a_{Y}$.

\section{B.2. Special Case 2: Heterogeneous Investors and Permanent Market Closure}

Next, we consider another special case in which investors are heterogeneous in their private investments and there are gains from trade, but there is no aggregate uncertainty in private investment opportunities. This case allows us to analyze in closed form the discrete changes in investors' hedging position at the close and the resulting time-variation in stock price.

In particular, we let $\omega=1 / 2, \sigma_{1}=\sigma_{2}, \kappa_{12}=-1$, and $Y_{1,0}=Y_{2,0}$. Then, $Y_{1, t}=-Y_{2, t}$ and shocks to individual investors' private technologies cancel with each other at the aggregate level, leaving no aggregate uncertainty about private investment opportunities. Moreover, we let $T$ be finite and $N=\infty$. The stock market opens during $[0, T]$ and then closes permanently thereafter. The result is stated in Proposition 2.

Proposition 2: When $\omega=1 / 2, \sigma_{1}=\sigma_{2}=\sigma_{Y}, \kappa_{12}=-1, Y_{1,0}=Y_{2,0}$, and $N=\infty$, the economy has a unique linear equilibrium. The equilibrium stock price is

$$
P_{t}=F_{t}-\lambda_{0} \quad \forall t \in[0, T]
$$

with $\lambda_{0}=\bar{\lambda}_{00}+\left(\bar{\lambda}_{0}-\bar{\lambda}_{00}\right) e^{-r(T-t)}$, and the investors' stock holdings are

$$
\theta_{i, t}= \begin{cases}1-|\bar{h}| Y_{i, t}, & t \in[0, T) \\ 1-\left(\bar{\lambda} / \bar{\lambda}_{0}\right) Y_{i, T}, & t=T \\ \theta_{i, T}, & t>T,\end{cases}
$$

where $\bar{h} \equiv-\kappa_{D q} \sigma_{D} /\left(r \sigma_{q} \bar{\lambda}_{0}\right), 0<\bar{\lambda} / \bar{\lambda}_{0}<|\bar{h}|$, and $\bar{\lambda}_{00}, \bar{\lambda}_{0}$, and $\bar{\lambda}$ are defined in Proposition 1.

${ }^{19}$ At zero expected excess returns, the private technologies attract negative investments because investors use them to hedge the risk of their stock investments. As a result, the total risk of the stock becomes $\bar{\sigma}_{D}^{2}+\sigma_{F}^{2}$ instead of $\sigma_{D}^{2}+\sigma_{F}^{2}$. 
Facing different private technologies, investors trade in the stock market to hedge the risk of their private investments. Since the risk exposures of the two classes of investors are perfectly negatively correlated, so are their hedging needs. Consequently, their hedging trade does not move the stock price.

When the stock market closes, the economy enters autarchy and investors can no longer mutually insure the risk from their private investments. Similar to the case of homogeneous investors, each investor's shadow price of the stock covaries with his private investment opportunity. The additional volatility in the (shadow) price makes the stock riskier for each investor when the market closes. In particular, the risk discount reaches $\bar{\lambda}_{0}$ at the close, its value under no trading. ${ }^{20}$ Right before the close, the market is open and investors can trade the stock to mutually insure the risk from their private investments. However, the price of the stock cannot drop to its corresponding level in the case of the market being permanently open. As discussed earlier, such a discrete change in price is ruled out by arbitrage arguments. It can only decrease gradually as we move further away from the closure. Far before the closure, that is, $t \ll T$ (assuming $T \gg 1 / r$ ), the economy is close to the case of the market being permanently open. The risk discount on the stock is close to its value when $\sigma_{Y}=0: \lambda_{0} \approx \bar{\lambda}_{00}$. As $t$ approaches $T$ (from the left), $\lambda_{0}$ approaches $\bar{\lambda}_{0}$ from $\bar{\lambda}_{00}$.

Proposition 2 explicitly shows how investors change their stock positions discretely at $T$. Before market closure, investors trade in the same fashion as in the case of the market being permanently open. In addition to their fair share of the market (one share per capita), the two classes of investors take on opposite stock positions to hedge the risk from their private investments. Their trading behavior is not at all affected by the approaching closure. At the closure, however, they discontinuously cut back their hedging positions to avoid the high risk of overnight positions. Note that $|\bar{h}|$ increases with $a_{Y}$ (since $\lambda_{0}$ decreases with $a_{Y}$ ). Thus, for more transitory private technological shocks, investors take larger hedging positions during the day but make larger cuts at the close.

\section{B.3. The General Case}

We now turn to the general case. The intuition from the special cases gives the following picture. Market closures give rise to time variation in investors' hedging trade. In particular, they discretely reduce their hedging positions at the close. As a result, the stock price is on average lower and less sensitive to investors' private technology shocks around the close than at the open.

In the following discussions, we use numerical examples to illustrate the predictions of the model. We choose "reasonable" parameter values. For example, the daily interest rate is set at 0.1 percent, the daily volatility of

${ }^{20}$ When $Y_{i, T}=0$, for example, $\theta_{i, T}=1$, and (from Proposition 1) investor $i$ 's shadow price of the stock is $P_{i, t}^{*}=F_{t}-\bar{\lambda}_{0}-\bar{\lambda} Y_{i, t}$ for $t>T$. At the closure point, the market price equals the shadow prices, which is $F_{T}-\bar{\lambda}_{0}$. Thus, $P_{T}=F_{T}-\bar{\lambda}_{0}$. For arbitrary values of $Y_{i, T}$, the intuition is the same. 
share returns at 0.135 , and the daily excess share return at $0.01 .^{21}$ For the remaining degrees of freedom, the choice for the parameter values is quite arbitrary since the qualitative features of the results are not sensitive to the particular values. In particular, we have set $\sigma_{1}=\sigma_{2}$ and $\omega=1 / 2$ to maintain certain symmetry between the two classes of investors. Given that the equilibrium is periodic, in all our illustrations we only present the patterns during a single trading period. The opening and closing points are $\tau=0$ and $\tau=$ $T=0.5$, respectively. For easy comparison in our numerical illustrations throughout the paper, we use the following values for the other parameters as a benchmark: $\gamma=1000, \sigma_{D}=\sigma_{G}=0.08, \sigma_{q}=0.5, a_{Y}=0.25, \sigma_{1}=\sigma_{2}=7$, $\kappa_{D q}=0.5$, and $\kappa_{12}=0$.

Figure 2, Panel A, plots the intraday patterns of $\lambda_{0}$ and $\lambda_{1}=\lambda_{2} \equiv \lambda$ (due to symmetry between the two classes of investors). Clearly, $\lambda_{0}$ increases during the day, indicating a decreasing price over the day. The lower price at the close reflects the increase in risk to hold the stock overnight. $\lambda$ decreases during the day. Hence, the price is least sensitive to the investors' technological shocks at the close as they reduce their hedging trade.

The investors' intraday holdings of the stock are similar to those in the case of permanent market closure considered above. The symmetry between the two classes of investors implies $h_{1, \theta}^{(1)}=h_{2, \theta}^{(2)}=-h_{1, \theta}^{(2)}=-h_{2, \theta}^{(1)}$. Figure 2, Panel $\mathrm{B}$, plots $h \equiv h_{1, \theta}^{(1)}$ over a trading day. At the beginning of the day, investors actively trade the stock to hedge the risk of their private investments and the value of $h$ is high. At the end of the day, they drastically reduce their hedging trade and $h$ drops discretely, as denoted by a circle at $\tau=0.5$. The value of $h$ maintains more or less the same level after the opening because the effect of closure is not significant. As the closing point approaches, investors start to adjust their positions, but not necessarily in the same direction as their closing trades. As we have seen in the case of permanent closure (Section IV.B.2), investors can always wait until the close to establish their overnight position. Their trades before the close are affected by several factors. To fix ideas, let us focus on investor 1. Suppose that $Y_{1, t}>0$ and he is selling the stock to hedge. On the one hand, the expected decrease of $\lambda$ as the closure approaches gives rise to expected price appreciation. Thus, the cost of selling the stock to hedge increases, which tends to make investors hedge less. On the other hand, the lower level of $\lambda$ itself reduces the cost of selling stock to hedge and the decrease in price volatility makes the stock a more attractive hedging vehicle, which tends to make investors hedge more. (Additional dynamic effects are discussed in footnote 17.)

From the intraday stock price, we can calculate the mean and the variance of instantaneous stock returns. Figure 2, Panel C, shows that the mean excess share return $e$ is decreasing during the day. Since the variability in the underlying state variables is constant over time, the intraday variability of

\footnotetext{
${ }^{21}$ Since we use share returns in this paper, one should be careful in relating the return numbers here to the observed numbers on the rate of return (which is the return on one dollar instead of one share). We have not tried to calibrate the model to actual data, but it is possible (see, e.g., Campbell and Kyle (1993)).
} 


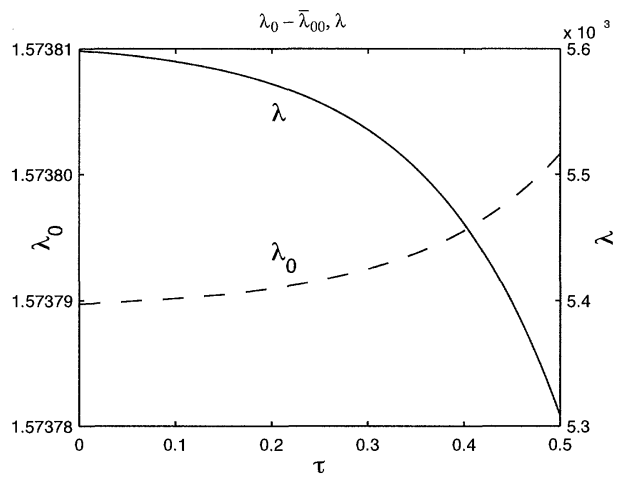

(a)

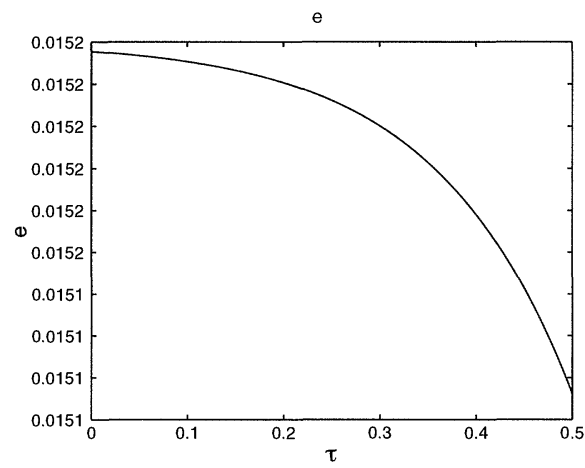

(c)

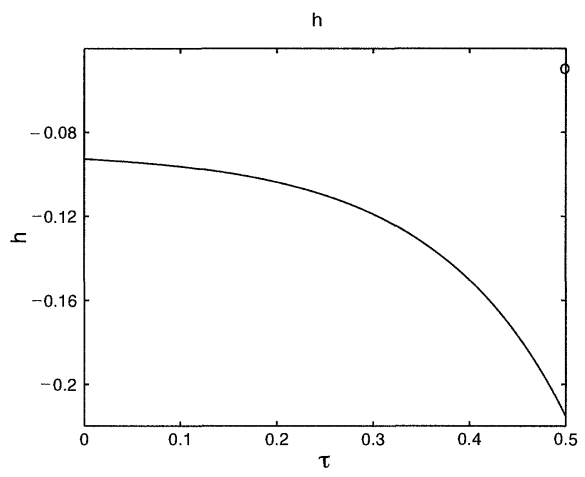

(b)

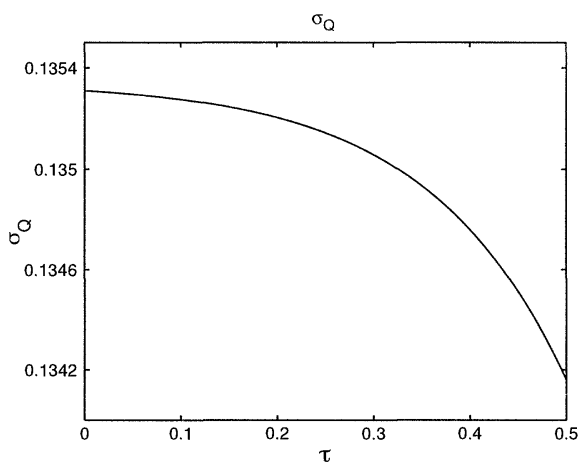

(d)

Figure 2. Intraday patterns of the stock price, holdings, and returns under symmetric information. The figures plot the time variation as follows: Panel $\mathrm{A}$, the risk premium, $\lambda_{0}$, and the sensitivity of the stock price to private technology shocks, $\lambda_{1}$; Panel $\mathrm{B}$, the sensitivity of stock positions to private technology shocks, $h$; Panel $\mathrm{C}$, the instantaneous mean excess share return on the stock, $e$; and Panel $\mathrm{D}$, the return volatility $\sigma_{Q}$, in the case of symmetric information. In Panel A the dashed line is $\lambda_{0}-\bar{\lambda}_{00}\left(\bar{\lambda}_{00}\right.$ is the risk discount with constant private investment opportunities) and its values are on the left $y$-axis while the solid line is $\lambda$ and its values are on the right $y$-axis. In Panel B the value of $h$ at the close is marked by a circle. The parameters are set at the following values: $T=0.5, N=0.5, \omega=0.5, \gamma=1000, r=0.001, \sigma_{D}=$ $0.08, a_{G}=0.85, \sigma_{G}=0.08, \sigma_{q}=0.5, a_{Y}=0.25, \sigma_{1}=\sigma_{2}=7, \kappa_{D q}=0.5, \kappa_{12}=0$.

the stock returns is determined purely by $\lambda$, the sensitivity of price to technological shocks. As $\lambda$ decreases during the day (see Panel A), the instantaneous price volatility also decreases during the day as Figure 2, Panel D, shows.

Given the reduction in investors' stock positions at the close, the trading activity at the close is abnormally high. Also, the information accumulation during the night gives rise to active trading at the open. Thus, abnormally high trading activities at the open and the close are the simple outcomes of periodic market closures in our model. 
Figure 2 illustrates the nature of the equilibrium using the benchmark parameter values. We now briefly discuss how the equilibrium changes as we change the values of different parameters. For a wide range of parameter values we have explored, the qualitative features of the equilibrium are the same as those shown in Figure 2. The quantitative features of course change with parameter values. A parameter of particular interest is $a_{Y}$. As discussed in the two special cases, a larger $a_{Y}$ gives more transitory shocks to private technologies, which lead to a higher level of hedging trade in the day and a larger drop at the close. As a result, a larger $a_{Y}$ leads to more time variation in the stock price. In particular, $\lambda_{0}$ increases more and $\lambda$ decreases more before the close. Other parameters can change the quantitative feature of the equilibrium as well. For example, when $a_{G}$ decreases, fluctuations in dividend growth become more persistent and the stock becomes riskier. As a result, the level of hedging decreases and so does $\lambda$. Moreover, the decrease in hedging positions at the close becomes larger, leading to more time variation in the stock price during the trading periods. The effect of varying $\sigma_{G}$ can be analyzed similarly. For brevity, we omit these results here.

\section{Discrete Simple Returns}

We now consider the discrete returns as defined in equation (8). In particular, the simple share returns from open to open, close to close, open to close, and close to open are given by $R^{o o}=R_{t_{k}, t_{k+1}}, R^{c c}=R_{n_{k}, n_{k+1}}, R^{o c}=$ $R_{t_{k+1}, n_{k+1}}$, and $R^{c o}=R_{n_{k}, t_{k+1}}$, respectively.

Given that $\lambda_{0}$ is a periodic function of time on $\mathcal{T}$, the expected simple return over a calendar day must be zero. ${ }^{22}$ Since the stock price is lower at the close than at the open, that is, $\lambda_{0}(0)<\lambda_{0}(T)$, the expected return over the day is lower than the expected return over the night.

Proposition 3. $\mathrm{E}\left[R^{o o}\right]=\mathrm{E}\left[R^{c c}\right]=0$. For $\lambda_{O}(0)<\lambda_{O}(T), \mathrm{E}\left[R^{o c}\right] \leq 0 \leq \mathrm{E}\left[R^{c o}\right]$.

Furthermore, $\lambda(0)>\lambda(T)$ implies that the stock price is more sensitive to the technological shocks at the open than at the close. Thus the open-to-open returns are more volatile than the close-to-close returns. ${ }^{23}$ Also, since the stock price linearly depends on the underlying state variables, the open-toclose returns and the close-to-open returns have the same volatility. Note that there is continuous trading from open to close but there is no trading

\footnotetext{
${ }^{22}$ In this paper, we have assumed that the stock dividend follows a stationary process and has a zero unconditional mean. Consequently, its price also follows a periodic-stationary process. Thus, simple returns over any number of calendar days have a zero unconditional mean. We can have a nonzero unconditional mean by adding a (constant) positive trend to the dividend process. The zero trend is assumed in the paper merely for simplicity. It is also worth pointing out that, conditionally, the return over a calendar day may be positive or negative, depending on the value of $G_{t}$ at the beginning of the day.

${ }^{23}$ The difference in the volatility of open-to-open and close-to-close returns also implies certain patterns in autocorrelation in returns. See Amihud and Mendelson (1987) for a discussion on this relation and the empirical evidence.
} 


\section{Table I}

\section{Discrete Stock Returns under Symmetric Information}

This table reports the unconditional mean and variance of various discrete returns produced by the model under symmetric information. $R_{o o}, R_{c c}, R_{o c}$, and $R_{c o}$ denote, respectively, the simple stock returns from a market open to the next open, from a market close to the next close, from a market open to the next close, and from a market close to the next open. The parameters are set at the following values: $T=0.5, N=0.5, \omega=0.5, \gamma=1000, r=0.001, \sigma_{D}=0.08, a_{G}=0.85$, $\sigma_{G}=0.08, \sigma_{q}=0.5, a_{Y}=0.25, \sigma_{1}=\sigma_{2}=7, \kappa_{D q}=0.5, \kappa_{12}=0$.

\begin{tabular}{lcc}
\hline Returns & Mean $\left(\times 10^{-4}\right)$ & Variance \\
\hline$R_{o o}$ & 0.00 & 0.0424 \\
$R_{c c}$ & 0.00 & 0.0421 \\
$R_{o c}$ & -0.12 & 0.0142 \\
$R_{c o}$ & 0.12 & 0.0142 \\
\hline
\end{tabular}

from close to open: This result suggests that under symmetric information, the volatility of discrete simple returns over a given period does not depend on whether or not there is trading within the period per se.

Proposition 4. For $\lambda(0)>\lambda(T), \operatorname{var}\left[R^{o o}\right]>\operatorname{var}\left[R^{c c}\right]$. For $T=N, \operatorname{var}\left[R^{o c}\right]=$ $\operatorname{var}\left[R^{c o}\right]$.

Table I reports the means and variances of discrete simple returns for the benchmark parameters used in Figure 2. Clearly, the mean return over market open is negative, the mean return over market close is positive, and the return volatility is the same over market close as over market open.

To summarize, we have shown in this section that investors reduce their hedging trade at the close, leading to time-variation in prices and returns. In particular, both the mean and volatility of stock returns decrease monotonically over the trading periods. The stock price also decreases during the trading periods, yielding higher returns over nontrading periods. The return volatility is the same for the periods of market open and the periods of market close. All these results are inconsistent with the empirical patterns. ${ }^{24}$ However, the open-to-open return is more volatile than the close-to-close return and trading activity is high at the close and open, which are consistent with the empirical findings.

\section{The Case of Asymmetric Information}

The existence of asymmetric information changes the time-variation of stock returns. In this section, we show that under asymmetric information, periodic market closures can generate a rich set of return patterns, including those consistent with the empirical findings.

${ }^{24}$ Kleidon and Werner (1996), and Chan et al. (1996) have found that intraday return volatility can decrease monotonically for stocks cross-listed on the exchanges in London, New York, and Tokyo. This would be consistent with our model, assuming that those stocks are often large stocks and their trading is driven mostly by allocational reasons. 


\section{A. Equilibrium under Asymmetric Information}

For simplicity, throughout this section we let $\boldsymbol{a}_{U}=\mathbf{0}$ and $\boldsymbol{b}_{U}=\mathbf{0}$. Thus, the public signal after time 0 reveals no information. Class 1 investors now have full information about the economy, while class 2 investors only have partial information. Thus, in this section we also refer to class 1 investors as the informed investors and class 2 investors as the uninformed investors.

The general solution of equilibrium in Section III gives the following stock price:

$$
P_{t}=\hat{F}_{t}-\lambda_{0}-\lambda_{1} Y_{1, t}-\lambda_{2} Y_{2, t}+\lambda_{G}\left(G_{t}-\hat{G}_{t}\right) \quad \forall t \in \mathcal{T} .
$$

In the absence of informed investors, the stock price takes the form $P_{t}=\hat{F}_{t}$ $\lambda_{0}-\lambda_{2} Y_{2, t}$, where $\hat{F}_{t}$ gives the value of expected future payoffs as perceived by the uninformed investors and $\lambda_{0}+\lambda_{2} Y_{2, t}$ gives the risk discount. In the presence of the informed investors, they make speculative trades based on their private information-for example, when the uninformed investors underestimate the dividend growth, $G_{t}-\hat{G}_{t}>0$, and the informed investors purchase the stock in expectation of future appreciation in the value of the stock, thereby driving up its current price. This gives the term $\lambda_{G}\left(G_{t}-\hat{G}_{t}\right)$ in the stock price where $\lambda_{G}>0$. Additionally, the informed investors' hedging activities give the term $\lambda_{1} Y_{1, t}$ in the risk discount. ${ }^{25}$

From Lemma 3, investors' optimal stock holdings are as follows:

$$
\begin{aligned}
& \theta_{1, t}=h_{1, \theta}^{(0)}+h_{1, \theta}^{(1)} Y_{1, t}+h_{1, \theta}^{(2)} Y_{2, t}+h_{1, \theta}^{(3)}\left(G_{t}-\hat{G}_{t}\right) ; \\
& \theta_{2, t}=h_{2, \theta}^{(0)}+h_{2, \theta}^{(1)} \hat{Y}_{1, t}+h_{2, \theta}^{(2)} Y_{2, t} .
\end{aligned}
$$

As in the case of symmetric information, the first three terms in both the informed and uninformed investor's stock position correspond to, respectively, the unconditional, hedging, and market-making components of his stock position. For an informed investor (investor 1), however, the additional term, $h_{1, \theta}^{(3)}\left(G_{t}-\hat{G}_{t}\right)$, corresponds to his speculative trade, which is proportional to $G_{t}-\hat{G}_{t}$ (the difference between the true dividend growth and the uninformed investors' estimate). The coefficient $h_{1, \theta}^{(3)}$ characterizes the intensity of the informed investors' speculative trading. For an uninformed investor (investor 2), he cannot perfectly identify the informed investors' trading motives. His market-making trade is based only on his expectation about the

\footnotetext{
${ }^{25}$ Another way to interpret the stock price is to rewrite it as $P_{t}=\lambda_{G} G_{t}+\left[1 /\left(r+a_{G}\right)-\lambda_{G}\right] \hat{G}_{t}-$ $\left(\lambda_{0}+\lambda_{1} Y_{1, t}+\lambda_{2} Y_{2, t}\right)$. The first two terms then reflect, respectively, how the expectations of informed and uninformed investors on future stock payoffs affect the stock price. In particular, we expect $0<\lambda_{G}<1 /\left(r+a_{G}\right)$. Since the uninformed investors' expectation of the true dividend growth comes from regressing the realized dividends, it is less sensitive to the true growth.
} 
informed investors' hedging needs, which depends on $\hat{Y}_{1, t}$ instead of $Y_{1, t}$. Part of his market-making trade actually corresponds to the speculative trade of the informed investors, who are on the opposite sides of the trade.

As the market closes and reopens, the endogenous flow of information in the economy also changes and so does the degree of information asymmetry among investors. This time-variation in information asymmetry leads to rich patterns in stock returns. In what follows, we first analyze the time variation in information asymmetry generated by market closures and then examine the resulting return patterns.

\section{B. Time Variation in Information Asymmetry}

During the closure, market prices cease to provide information to the uninformed investors. As the informed investors continue to receive new private information, the information asymmetry between the two classes of investors increases over the night. At the open, trading resumes. The opening price partially reveals the private information of the informed investors accumulated over the closure, causing the level of information asymmetry to drop discretely. As trading continues during the day, more private information is revealed through the prices, and the level of information asymmetry may continue to decrease. At the close, two additional forces come into play. On the one hand, the informed investors cut back their hedging positions, which also unveils their speculative positions and their private information. This tends to speed the decrease in information asymmetry at the close. On the other hand, the informed investors also cut back their speculative positions due to the high risk over the closure. The reduction in speculative trade makes the stock price less revealing about existing private information. This tends to increase the information asymmetry at the close. The interaction between these two forces determines the time variation in information asymmetry around the close. ${ }^{26}$

We define the uninformed investors' conditional standard deviation of the dividend growth, $\delta \equiv\left\{\mathrm{E}_{2, t}\left[\left(G_{t}-\hat{G}_{t}\right)^{2}\right]\right\}^{1 / 2}$, as a measure of information asymmetry between the two classes of investors. The level and the time variation of information asymmetry depend on several factors, in particular on the nature of the $G_{t}$ process and $Y_{1, t}$ process, each characterized by its instantaneous volatility and mean-reversion coefficients $\left(\sigma_{G}, a_{G}\right.$ and $\sigma_{Y}, a_{Y}$, respectively), and the length of closure $N$.

Figure 3, Panel A, plots the time path of $\delta$ through the course of a calendar day for different values of $\sigma_{G}$ (the other parameters are set at the benchmark values, except that the fraction of class 1 investors is now $\omega=0.05$ instead of 0.5$)$. The time interval $(-0.5,0)$ corresponds to the night and $[0,0.5]$ corresponds to the following day. When $\sigma_{G}=0$, the dividend growth is con-

\footnotetext{
${ }^{26}$ The behavior of the equilibrium near the close depends on the competitive assumption, under which the informed investors revise their hedging positions without taking into account the fact that revisions also reveal their speculative positions. In a noncompetitive model, the informed investors would take into account the information impact of their hedging activities, and optimally trade off the benefits and costs of cutting back hedging positions at the close.
} 


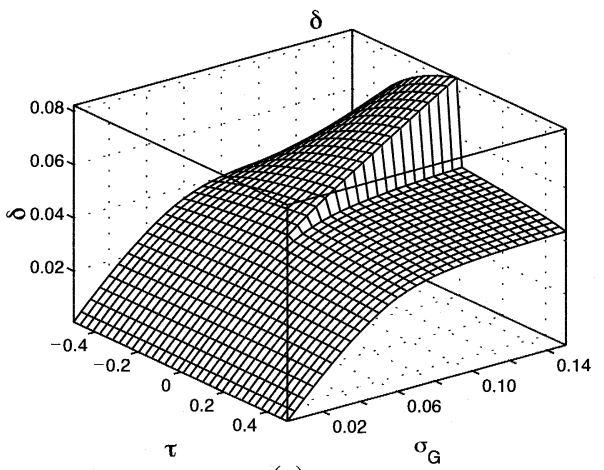

(a)

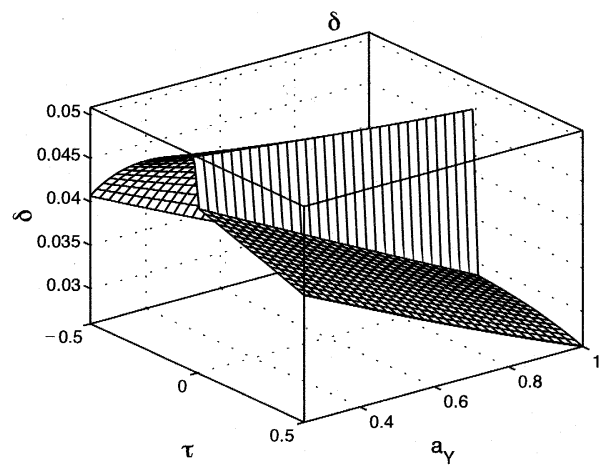

(c)

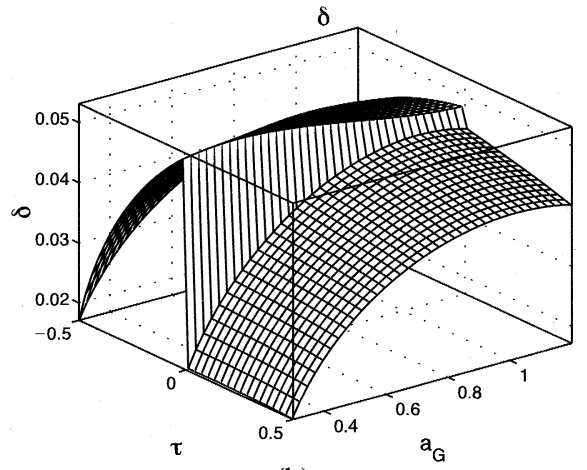

(b)

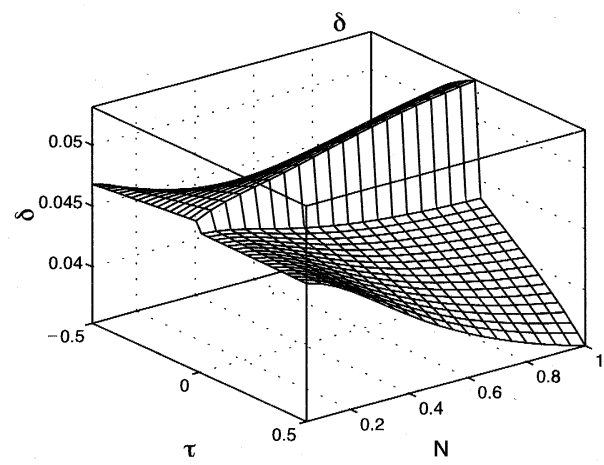

(d)

Figure 3. Intraday patterns of information asymmetry. The figures plot the time variation of information asymmetry, $\delta$, for different values of: Panel $\mathrm{A}$, the volatility of dividend growth rate, $\sigma_{G}$; Panel $\mathrm{B}$, the persistence of dividend growth rate, $a_{G}$; Panel $\mathrm{C}$, the persistence of private investment opportunities, $a_{Y}$; and Panel D, the length of the night. In Panel A, $a_{G}=$ $0.85, a_{Y}=0.25$, and $N=0.5$. In Panel B, $\sigma_{G}=0.08, a_{Y}=0.25$, and $N=0.5$. In Panel $\mathrm{C}, \sigma_{G}=$ $0.08, a_{G}=0.85$, and $N=0.5$. In Panel D, $\sigma_{G}=0.08, a_{G}=0.85, a_{Y}=0.25$. The other parameters are set at the following values: $T=0.5, \omega=0.5, \gamma=1000, r=0.001, \sigma_{D}=0.08, \sigma_{q}=0.5, \sigma_{1}=$ $\sigma_{2}=7, \kappa_{D q}=0.5, \kappa_{12}=0$.

stant and $\delta=0 \forall t$. As $\sigma_{G}$ increases, the unconditional uncertainty of $G_{t}$, given by $\mathrm{E}\left[G_{t}^{2}\right]=\sigma_{G}^{2} /\left(2 a_{G}\right)$, increases, and so does the overall level of $\delta$. For $\sigma_{G}>0, \delta$ varies over time. Due to the periodicity of equilibrium, $\delta$ takes the same value at $\tau=-0.5$ and 0.5 . Clearly, $\delta$ increases during the night as the uninformed investors lose the market price as a source of information while the informed investors accumulate more private information. It drops discretely as the market reopens and continues to decrease as trading continues. Comparing the time path of $\delta$ for different values of $\sigma_{G}$ in Panel A further shows that for larger values of $\sigma_{G}, \delta$ increases faster over the night, reflecting more accumulation of private information, and it drops more at the open as the opening price reveals a larger amount of the private information accumulated over the night. 
Fluctuations in $G_{t}$ (from its unconditional mean) have a half-life of $1 / a_{G}$ and are more persistent for smaller values of $a_{G}$. Figure 3 , Panel B, illustrates how the persistence of $G_{t}$ fluctuations changes the time pattern of $\delta$. Higher persistence in $G_{t}$ fluctuations implies that (a) the unconditional uncertainty about $G_{t}$ is higher, (b) values of $G_{t}$ at different times are more correlated, and (c) the private information of the informed investors on $G_{t}$ is longer-lived. The higher unconditional uncertainty tends to increase the overall level of informational uncertainty. In particular, $\delta$ increases faster during the night (as the market closes) and reaches a higher level right before the open. But, it goes through a larger drop at the open. As values of $G_{t}$ at different times become more correlated, the previous closing price contains more information about the value of $G_{t}$ at the open. Combined with the previous closing price, the opening price reveals more information about the value of $G_{t}$, leading to a larger decrease in $\delta$ at the open.

Although $\delta$ usually keeps decreasing as trading continues after the open, its behavior around the close depends on the half-life of the informed investors' private information on $G_{t}$. For highly transitory $G_{t}$ fluctuations, the informed investors' private information (on $G_{t}$ ) becomes very short-lived and its value dissipates fast over time. In particular, their private information on $G_{t}$ at the close has little value in forecasting its value at the next open and the expected payoff on overnight speculative positions is small. In this case, informed investors would cut most of their speculative position at the close and the stock price then reflects little of their private information, causing $\delta$ to increase as the closure approaches. This effect from the reduction in their speculative trade is opposite to the effect from the reduction in their hedging trade, which causes $\delta$ to decrease faster before the close. The actual pattern of $\delta$ depends on which one of these two effects dominates. ${ }^{27}$ As shown in Figure 3, Panel B, $\delta$ decreases monotonically during the day and the decrease speeds up toward the close. However, for very large values of $a_{G}$ (not shown in the figure), the reduction of their speculative position at the close has a stronger effect; $\delta$ decreases faster earlier in the day, slows down as the close approaches, and could even start to increase before the close (see Hong and Wang (1998) for more discussions).

The degree of information asymmetry also depends on the nature of the $Y_{1, t}$ process, which drives the informed investors' hedging trades and gives rise to the "noise" in the price of the stock as a signal about its future payoffs. For larger values of $\sigma_{1}, Y_{1, t}$ becomes more volatile and the informed investors trade more for their hedging needs. More noise from their hedging trade leads to less informative prices about stock payoffs and a higher level of information asymmetry. The persistence in the informed investors' hedging needs, as measured by $a_{Y}$, determines the serial correlation in the noise. With low serial correlation in the noise, neighboring prices are close to independent signals (about $G_{t}$ ), and they tend to reveal more of the informed investors' private information on $G_{t}$. With high serial correlation in the noise, neighboring prices are more

\footnotetext{
27 There are also other indirect effects of changing $a_{G}$ on $\delta$. For example, as $a_{G}$ decreases, $F_{t}=$ $1 /\left(r+a_{G}\right) G_{t}$ becomes more volatile (keeping other things the same). Thus, the stock becomes riskier as a hedging vehicle and the level of hedging decreases, and so does the level of $\delta$.
} 
correlated as signals, and they tend to reveal less information on $G_{t}$. Thus, for larger values of $a_{Y}$, a sequence of market prices tends to be more informative about the true value of $G_{t}$. Figure 3, Panel C, shows the time variation of $\delta$ for different values of $a_{Y}$. Clearly, for larger values of $a_{Y}$, the drop in $\delta$ at the open is larger since the prices at the open and previous close provide less-correlated signals about $G_{t}$. The level of $\delta$ during the day is also lower. Furthermore, for larger values of $a_{Y}$, investors cut more of their hedging positions at the close because their hedging need is more transitory but the positions have to be held overnight. More reduction in informed investors hedging positions can reveal more of their private information about stock payoffs. Thus, $\delta$ decreases faster toward the end of the day for larger values of $a_{Y}$.

Another important parameter in determining the time variation of information asymmetry is $N$, the length of the night. As we increase the length of the night, it has three effects. First, a longer night allows more overnight accumulation of private information. Thus, we may see a higher level of information asymmetry at the open than later in the day, giving rise to more time variation in the day. Next, a longer night makes overnight hedging positions riskier. As the informed investors cut their hedging positions at the close, prices become more informative about their speculative trade and private information, causing the information asymmetry to decrease faster at the close. Third, a longer night also makes overnight speculative positions riskier. It further reduces the expected gains since the private information at the close becomes less valuable in predicting the next opening price (the same effect as shortening the half-life of private information). Thus, the informed investors speculate less with overnight positions, which tends to increase the information asymmetry as the close approaches. Figure 3, Panel $\mathrm{D}$, shows the time variation of $\delta$ for different values of $N$.

In the above discussion, we have focused mainly on the absolute level and the time variation of information asymmetry over time. We now want to compare the level of information asymmetry under periodic market closures ( $N$ is finite) with its level in absence of any closures $(N=0)$. In the current model, the uninformed investors rely on market prices to extract information about the true dividend growth. When the stock market closes, they lose this important source of information. As a result, we would expect the level of information asymmetry to be higher when the market is closed more often. This, however, is only part of the story. As discussed earlier, market closures tend to reduce investors' hedging trade (especially at the close) and the "noise" in the price it generates. Consequently, prices become more informative about future payoffs, and the overall level of information asymmetry may decrease when the market is closed more often. In other words, closing the market periodically can actually make the prices more informative and reduce the information asymmetry about future payoffs. Figure 4 shows that for some parameter values the level of $\delta$ with periodic closures (when $N \neq 0$ ) can indeed lie uniformly below its level without closures (when $N=0$ ). This result stands in contrast to those from models of noisy rationalexpectations equilibrium in which allocational trading is exogenously specified, independent from market closures (see, e.g., Slezak (1994)). 


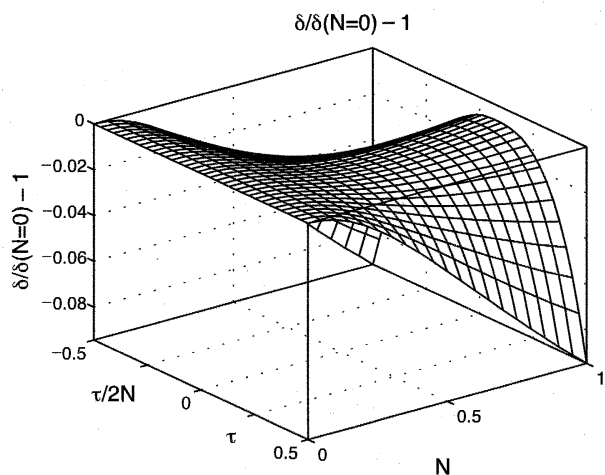

(a)

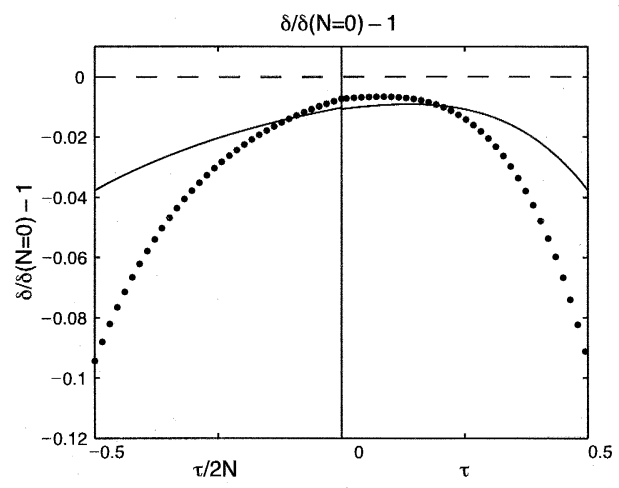

(b)

Figure 4. Intraday patterns of information asymmetry relative to its level in absence of closures. The figures plot the time variation of information asymmetry, $\delta$, relative to its level in absence of closures $(N=0)$, for different values of the length of the night, $N$. In Panel B the dashed line is for $N=0$, the solid line for $N=0.5$, and the dotted line for $N=1$. The other parameters are set at the following values: $T=0.5, \omega=0.05, \gamma=1000, r=0.001, \sigma_{D}=0.08$, $a_{G}=1, \sigma_{G}=0.025, \sigma_{q}=0.5, a_{Y}=0.25, \sigma_{1}=\sigma_{2}=7, \kappa_{D q}=0.5, \kappa_{12}=0$.

\section{Time Variation in Trading and Returns}

We now examine the effect of time-varying information asymmetry on equilibrium prices, trading strategies, and return distributions. We first illustrate the nature of this effect using a particular set of parameter values and then examine the results for other parameter values.

\section{C.1. Stock Price Coefficients}

Figure 5 illustrates the time pattern of price coefficients $\lambda_{0}, \lambda_{1}, \lambda_{2}$, and $\lambda_{G}$ for the benchmark parameters. The coefficient $\lambda_{0}$ gives the mean risk discount of the stock. After the open, more private information is revealed through trading; hence the uninformed investors' uncertainty about the stock's future payoffs decreases. In equilibrium, a lower risk discount is required, and $\lambda_{0}$ decreases after the market opens. Near the close, the risk discount decreases faster due to faster information revelation as the informed investors reduce their hedging trade (see Figure 3). $\lambda_{1}$ decreases during the day as in the case of symmetric information, reflecting the anticipated reduction in the informed investors' hedging positions at the close. $\lambda_{2}$ now exhibits a U-shaped pattern. $\lambda_{G}$ reflects the impact of the informed investors' speculative trade on the equilibrium price. It increases monotonically during the day, indicating that the stock price is more informative about the dividend growth at the end of the day than at the beginning. 


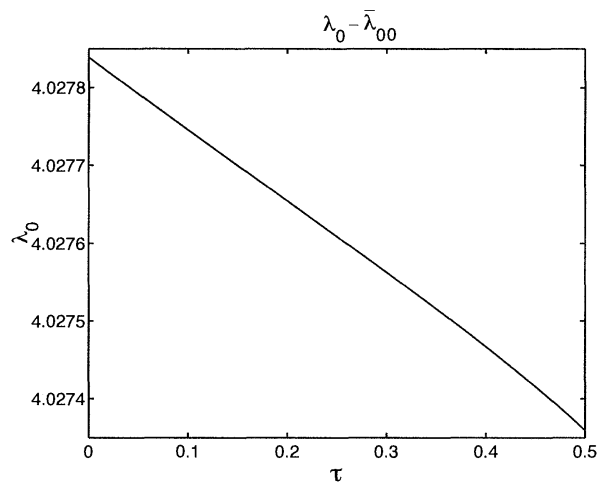

(a)

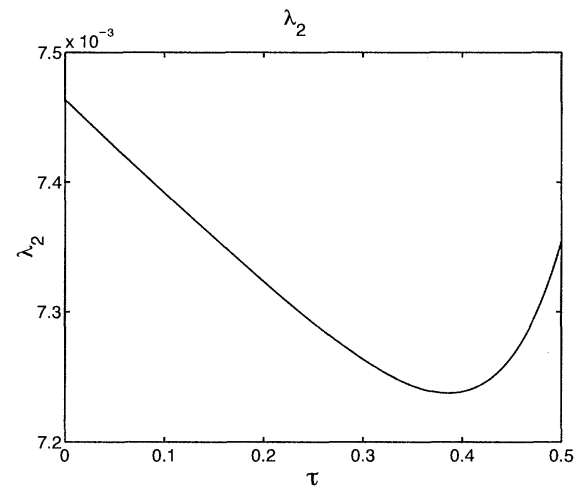

(c)

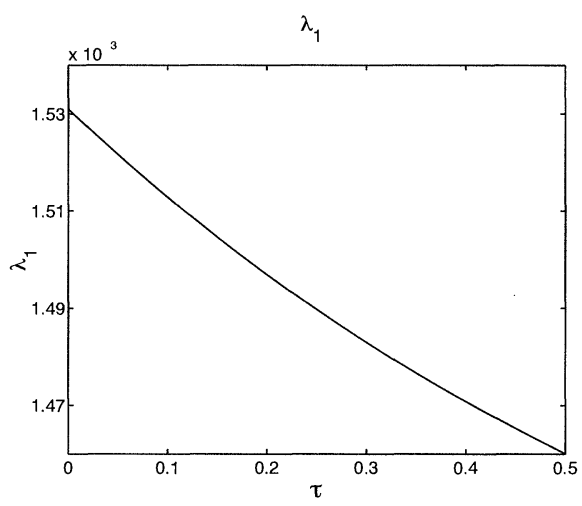

(b)

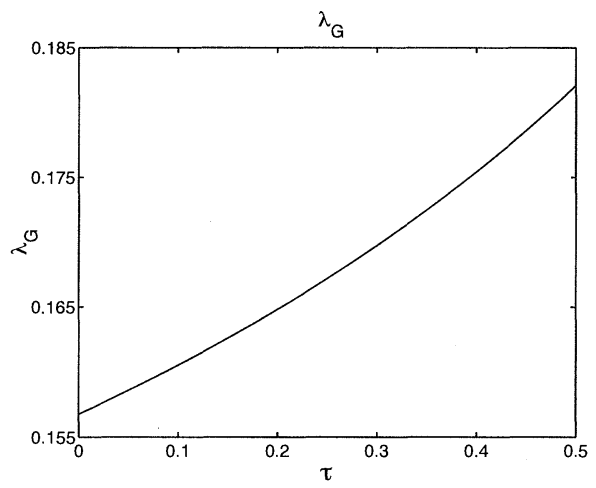

(d)

Figure 5. Intraday patterns of the stock price under asymmetric information. The figures plot the time variation of the risk premium, $\lambda_{0}$, and the sensitivities of the stock price to changes in the private technologies of class 1 and class 2 investors, and the dividend growth rate, $\lambda_{1}, \lambda_{2}$, and $\lambda_{G}$, respectively. The parameters are set at the following values: $T=0.5, N=$ $0.5, \omega=0.5, \gamma=1000, r=0.001, \sigma_{D}=0.08, a_{G}=0.85, \sigma_{G}=0.08, \sigma_{q}=0.5, a_{Y}=0.25, \sigma_{1}=$ $\sigma_{2}=7, \kappa_{D q}=0.5, \kappa_{12}=0$.

\section{C.2. Investors' Trading Strategies}

In order to better understand the time variation in price, let us now examine the investors' trading behavior in equilibrium. For brevity, we focus on the informed investors. The trading behavior of the uninformed investors in equilibrium can be inferred from the market clearing condition of equation (25).

Figure 6 illustrates the different components of the informed investors' stock holdings, again for the benchmark parameters. Panel A gives an informed investor's mean stock position in excess of his market share. Since the informed investors have better information about the dividend growth 


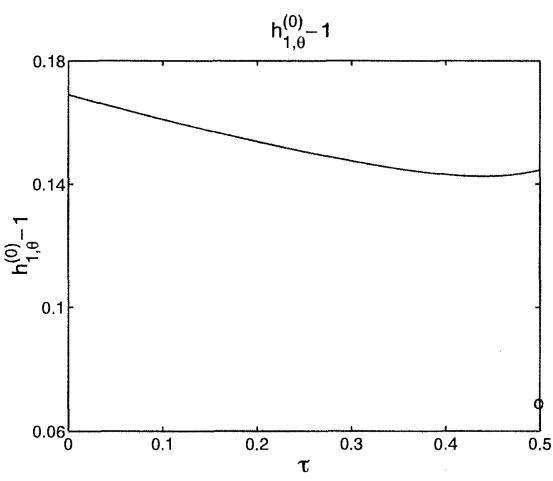

(a)

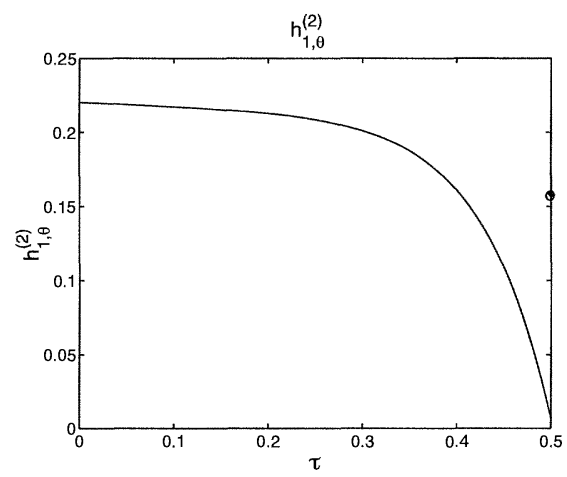

(c)

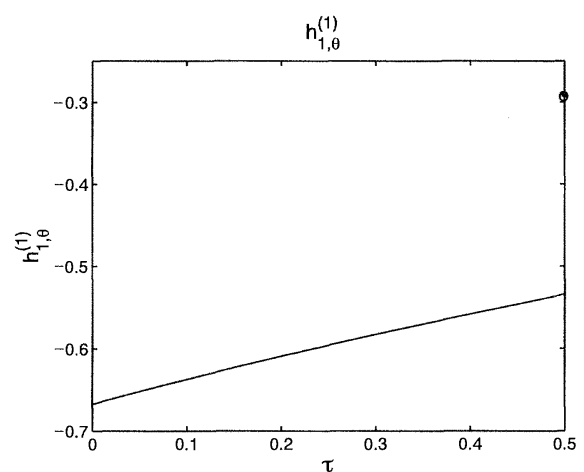

(b)

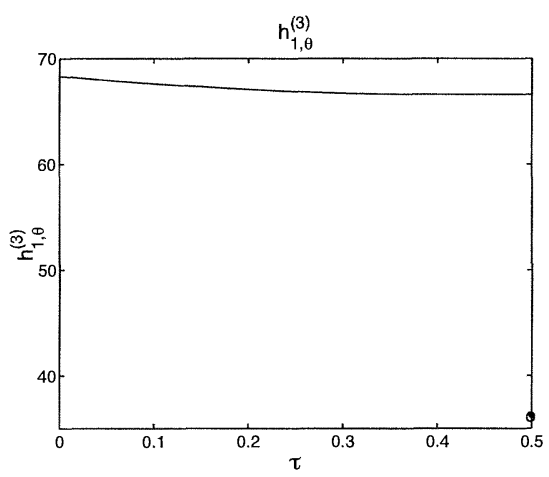

(d)

Figure 6. Intraday patterns of an informed investor's stock holding under asymmetric information. The figures plot the time variation of an informed investor's mean stock holding $h_{1, \theta}^{(0)}$ and the sensitivities of his holding to changes in the private investment opportunities of class 1 and class 2 investors, and the dividend growth-rate, $h_{1, \theta}^{(1)}, h_{1, \theta}^{(2)}$ and $h_{1, \theta}^{(3)}$, respectively. Their values at the close $(\tau=T)$ are indicated by circles. The parameters are set at the following values: $T=0.5, N=0.5, \omega=0.5, \gamma=1000, r=0.001, \sigma_{D}=0.08, a_{G}=0.85, \sigma_{G}=0.08$, $\sigma_{q}=0.5, a_{Y}=0.25, \sigma_{1}=\sigma_{2}=7, \kappa_{D q}=0.5, \kappa_{12}=0$.

than the uninformed investors, they perceive lower risk for holding the stock, thereby holding more stock shares on average $\left(h_{1, \theta}^{(0)}>1\right)$. Their excess stock positions tend to decrease after the market opens as information asymmetry decreases, and drop discretely at the close. ${ }^{28}$

${ }^{28}$ Another factor in determining the average stock holdings of the two classes of investors is the allocation of risk among them. For Figure 6, the informed investors are only a small fraction of the market (i.e., $\omega=0.05$ ). With $\sigma_{1}=\sigma_{2}$, each individual investor faces similar nontraded risk. But the nontraded risk of uninformed investors is less diversifiable because they are the majority and they face the same risk. In other words, they bear most of the aggregate nontraded risk. Given the positive correlation between nontraded income and stock payoff, the uninformed investors are less willing to hold the stock. Both of these factors tend to yield $h_{1, \theta}^{(0)}-1>0$. 
The informed investors' hedging activity, as characterized by $h_{1, \theta}^{(1)}$, is shown in Figure 6, Panel B. It behaves in a fashion similar to the case of symmetric information. The informed investors actively trade in the stock in response to changes in their private investments, but reduce their positions abruptly at the close. The coefficient $h_{1, \theta}^{(2)}$ characterizes the market-making activity of informed investors, which in turn reflects the intensity of uninformed investors' hedging activities. Factors affecting the informed investors' hedging activities are also affecting the uninformed investors. Additionally, the continuous revelation of information through the day reduces the risk of taking stock positions, thereby increasing the hedging activities of the uninformed. Also, as the market closes, market prices cease to be a source of information about the stock's future payoffs, and the uninformed investors rely solely on the information contained in realized dividends and public announcements. Consequently, changes in their expectations of dividend growth, now completely driven by dividend realizations, become more correlated with shocks to their nontraded income. Thus, the stock is perceived to be a better hedging vehicle during the night than during the day, causing uninformed investors to increase their hedging positions at the close. Both of these additional factors tend to increase the uninformed investors' hedging activity around the close as Figure 6, Panel C shows, thereby increasing $\lambda_{2}$ (see Figure 5, Panel C).

Finally, we consider the speculative activities of the informed investors. The informed investors speculate actively during the day. At the close, they abruptly reduce their speculative positions because the risk of an overnight speculative position is high and its expected payoff is low as discussed earlier. The time variation of the informed investors' speculative trade is illustrated by $h_{1, \theta}^{(3)}$ in Figure 6, Panel D.

\section{C.3. Time Pattern of Returns}

Given the price process, we can now calculate the intraday patterns in the mean return and return volatility. Two factors are driving the time variation in the return-generating process, both associated with market closures. The first factor is the time variation in investors' hedging demand. In particular, all investors reduce their hedging demand at the close, causing the stock price to decrease and become less responsive to investors' technological shocks as the close approaches. Consequently, the mean return and volatility decrease during the day. The second factor is the time variation in information asymmetry. As trading stops during the night, the information asymmetry on the stock (as measured by $\delta$ ) increases over time. But as trading resumes, the information asymmetry decreases over time as Figure 3 shows. Consequently, the stock price increases during the day as a smaller premium is required on the stock by the uninformed investors. Also, the price becomes more volatile because it reflects more information about the stock's future payoffs. Thus, the time variation in information asymmetry tends to cause the mean and volatility of stock returns to increase during the day. The 


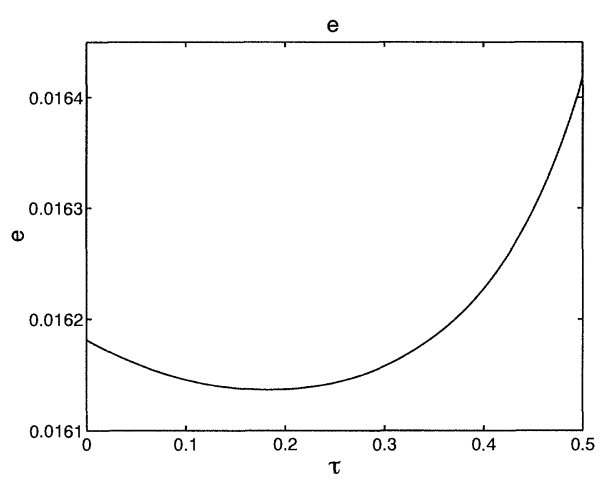

(a)

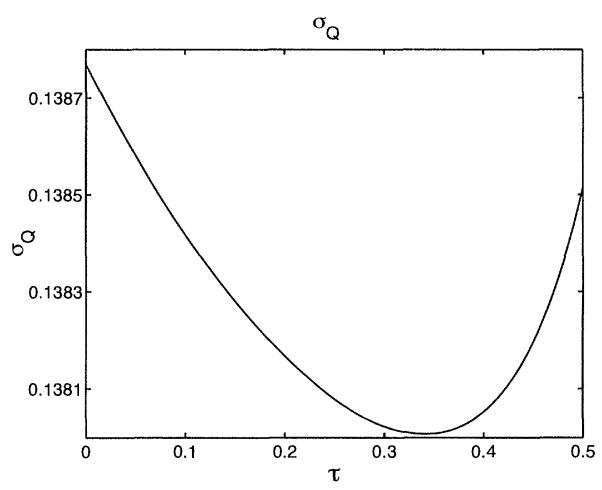

(b)

Figure 7. Intraday patterns of stock returns under asymmetric information. The figures plot the time variation of, Panel $\mathrm{A}$, instantaneous mean excess share return, $e$, and Panel $\mathrm{B}$, the return volatility, $\sigma_{Q}$. The parameters are set at the following values: $T=0.5, N=0.5, \omega=$ $0.5, \gamma=1000, r=0.001, \sigma_{D}=0.08, a_{G}=0.85, \sigma_{G}=0.08, \sigma_{q}=0.5, a_{Y}=0.25, \sigma_{1}=\sigma_{2}=7, \kappa_{D q}=$ $0.5, \kappa_{12}=0$.

resulting time pattern of stock returns is determined by the interaction between these two factors. In the extreme case when the effect of time-varying hedging demand dominates throughout the day, both the return and its volatility decrease monotonically as in the case of symmetric information. In another extreme case, when the effect of time-varying information asymmetry dominates throughout the day, the return and volatility increase monotonically during the day.

A more interesting case arises when the effect of time-varying hedging demand dominates early in the day and the effect of time-varying information asymmetry dominates later in the day. In this case, the U-shaped intraday patterns emerge for both the mean and volatility of returns. Figure 7 illustrates these patterns for the benchmark parameters. In particular, both the mean and the volatility of instantaneous stock returns are increasing over time right before the close, instead of decreasing as under symmetric information. These patterns are consistent with those observed empirically.

It is important to note that even though the U-shaped patterns occur for a wide range of parameter values, they are by no means the only patterns in addition to the simple, monotonic patterns. For some parameter values, we have also found inverted U-shaped patterns for both the mean and volatility of stock returns. Needless to say, these patterns depend on different parameters of the model, such as those characterizing the $G_{t}$ process and $Y_{i, t}$ process and the length of the day and night. We refer the readers to our working paper (Hong and Wang (1998)) for more discussions on various possible patterns of return and volatility. 


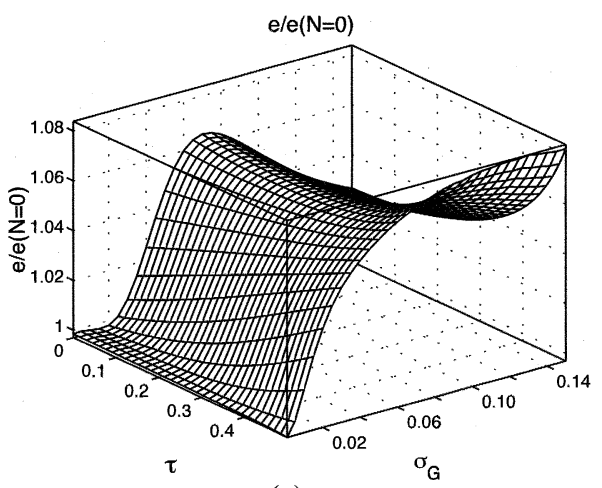

(a)

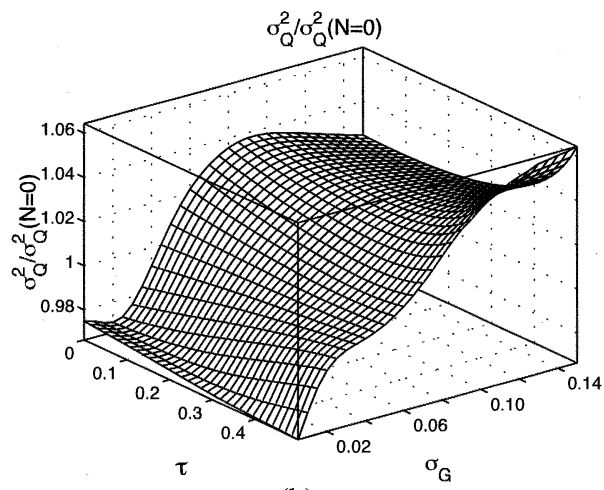

(b)

Figure 8. Intraday patterns of returns under asymmetric information for different volatility of dividend growth rate. The figures plot the time variation of, Panel A, the instantaneous mean excess share return, $e$, and, Panel $\mathrm{B}$, the return volatility, $\sigma_{Q}$, for different values of the volatility of dividend growth rate, $\sigma_{G}$. For ease of comparison, the mean return and return volatility are normalized by their respective values in the absence of closures, $e(N=0)$ and $\sigma_{Q}^{2}(N=0)$. Other parameters are set at the following values: $T=0.5, N=0.5$, $\omega=0.05, \gamma=1000, r=0.001, \sigma_{D}=0.08, a_{G}=0.85, \sigma_{q}=0.5, a_{Y}=0.25, \sigma_{1}=\sigma_{2}=7, \kappa_{D q}=0.5$, $\kappa_{12}=0$.

\section{C.4. Further Discussions}

We now examine how intraday return patterns may change as model parameters change. For brevity, we focus in this paper only on a subset of parameters, including those affecting the $G_{t}$ and $Y_{i, t}$ processes and the length of closures, and a limited range of their values (see Hong and Wang (1998) for more details on the effect of a larger set of parameters on return patterns and a wider range for their values).

1. Varying $\sigma_{G}$ and $a_{G}$. As discussed in Section V.B, $\sigma_{G}$ and $a_{G}$ are important in determining the time-variation of information asymmetry. Consequently, they affect the equilibrium return process. Figure 8 shows how the stock return and its volatility change with $\sigma_{G}$. For small values of $\sigma_{G}$, the degree of information asymmetry is small as shown in Figure 3, Panel A, and so is its effect on the equilibrium. In this case, the return patterns are similar to those under symmetric information: both the mean return and return volatility decrease monotonically during the day. For large values of $\sigma_{G}$, the effect of time-varying information asymmetry dominates. The mean return and return volatility tend to increase as information asymmetry diminishes over the day. For the middle range of $\sigma_{G}$, the effect of time-varying hedging trade tends to be more important earlier in the day, and the effect of time-varying information asymmetry tends to be more important later in the day. Thus, the mean return and return volatility both exhibit U-shaped intraday patterns. 


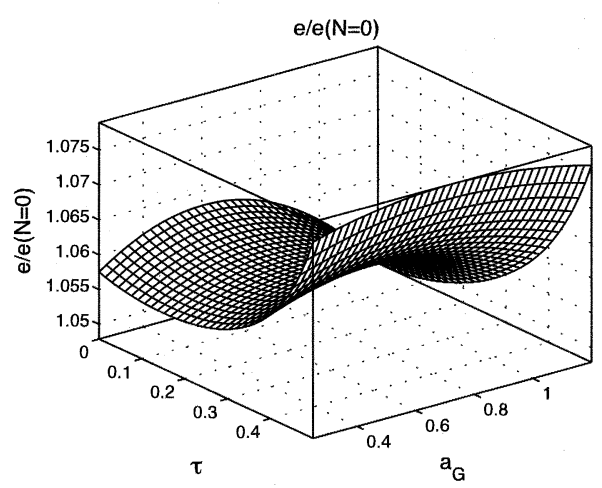

(a)

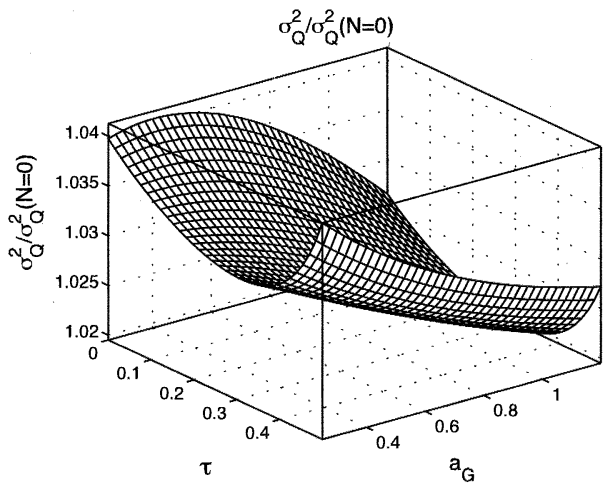

(b)

Figure 9. Intraday patterns of returns under asymmetric information for different persistence of dividend growth rate. The figures plot the time variation of, Panel $\mathrm{A}$, the instantaneous mean excess share return, $e$, and, Panel $\mathrm{B}$, the return volatility, $\sigma_{Q}$, for different values of the persistence of dividend growth rate, $a_{G}$. For ease of comparison, the mean return and return volatility are normalized by their respective values in the absence of closures, $e(N=0)$ and $\sigma_{Q}^{2}(N=0)$. Other parameters are set at the following values: $T=0.5, N=0.5$, $\omega=0.05, \gamma=1000, r=0.001, \sigma_{D}=0.08, \sigma_{G}=0.08, \sigma_{q}=0.5, a_{Y}=0.25, \sigma_{1}=\sigma_{2}=7, \kappa_{D q}=0.5$, $\kappa_{12}=0$.

Figure 9 illustrates how intraday return patterns change with $a_{G}$. For most values of $a_{G}$, the U-shaped patterns appear for both the mean return and the return volatility. However, as $a_{G}$ becomes large, the intraday variation of information asymmetry increases as shown in Figure 3, Panel B. Thus, the effect of information asymmetry becomes more important and so the increasing trend in the mean and the volatility become more prominent (particularly for the mean).

2. Varying $a_{Y}$. Figure 10 shows the intraday patterns in mean stock return (Panel A) and return volatility for different values of $a_{Y}($ Panel B). For most values of $a_{Y}$, the patterns are U-shaped. However, as $a_{Y}$ increases, the initial decrease in both the mean and the volatility becomes less significant. As discussed earlier, the U-shaped patterns appear when the hedging effect is more important earlier in the day and the information effect is more important later in the day. Moreover, the hedging effect is mainly driven by the decrease in investors' hedging demand at the close. When $a_{Y}$ becomes larger, the hedging demand at the close is less related to the hedging demand at the open. The hedging effect tends to be less important earlier in the day. Also, faster revelation of private information through the sequence of prices makes the information effect more important. Thus, the upward trend in the mean return and the return volatility becomes more important as $a_{Y}$ increases. (Moreover, as $a_{Y}$ increases, the cutback at the close is larger, which tends to increase the overall size of the hedging effect at the close.) 


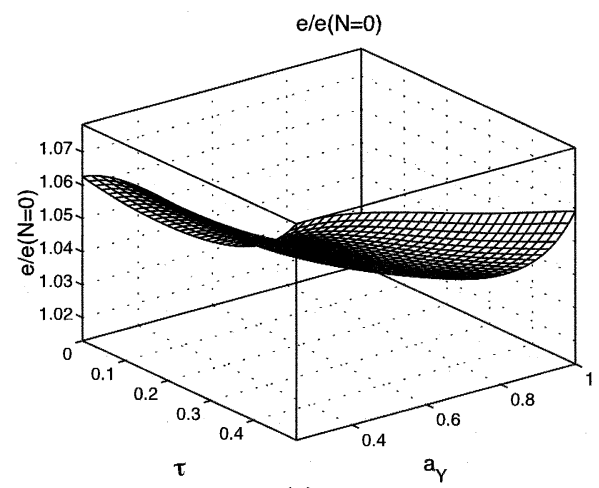

(a)

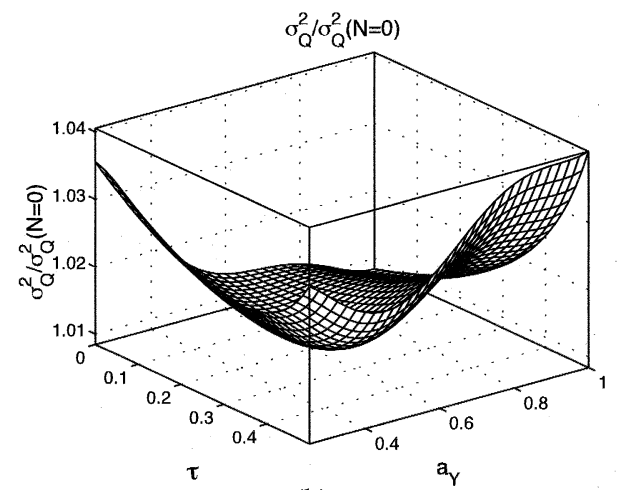

(b)

Figure 10. Intraday patterns of returns under asymmetric information for different persistence of investors' private investment opportunities. The figures plot the time variation of, Panel $\mathrm{A}$, the instantaneous mean excess share return, $e$, and, Panel $\mathrm{B}$, the return volatility, $\sigma_{Q}$, for different values of the persistence of investors' private investment opportunities, $\sigma_{G}$. For ease of comparison, the mean return and return volatility are normalized by their respective values in the absence of closures, $e(N=0)$ and $\sigma_{Q}^{2}(N=0)$. Other parameters are set at the following values: $T=0.5, N=0.5, \omega=0.05, \gamma=1000, r=0.001, \sigma_{D}=0.08, a_{G}=0.85$, $\sigma_{G}=0.08, \sigma_{q}=0.5, \sigma_{1}=\sigma_{2}=7, \kappa_{D q}=0.5, \kappa_{12}=0$.

3. Varying $N$ and $T$. We now examine how the lengths of night, $N$, and day, $T$, affect the interaction between market open and close. As discussed earlier, changing the length of night changes the risk of carrying overnight positions. For larger $N$, investors reduce their hedging positions more dramatically at the close, giving rise to larger intraday variation in both hedging trade and information asymmetry (see Figure 3, Panel D). ${ }^{29}$ As the length of the day $(T)$ changes, the effect of the closure on the prices and returns around the open also changes. For example, larger values of $T$ tend to diminish the effect of reduction in hedging trade on returns around the open. For smaller values of $T$, however, both the effect of time-varying hedging trade and information asymmetry are squeezed into a small interval, leading to less time variation and relatively simpler patterns in returns.

Figure 11 shows how the intraday patterns of return and volatility change as we change $T$ and $N$, holding the total length of a calendar day $T+N$ constant (so that the total amount of risk per calendar day is fixed). Note that in Figure 11, we only show the range of small $N$ (from 0.1 to 0.2 ). This is motivated by the observation that in economic time, measured by the amount of information arrival, a night may only be a small fraction of a day. As $N$ approaches zero (and $T$ approaches one), we approach the case of the

${ }^{29}$ We discussed in Section V.B that longer nights also make overnight speculative positions riskier and less profitable. Thus, as $N$ increases, investors' speculative trades tend to decrease as well, which makes prices less informative. For the cases we consider here, the effect from decreasing hedging trade dominates. 


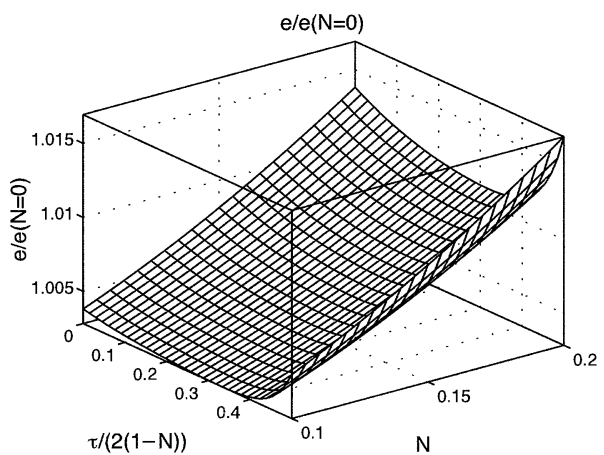

(a)

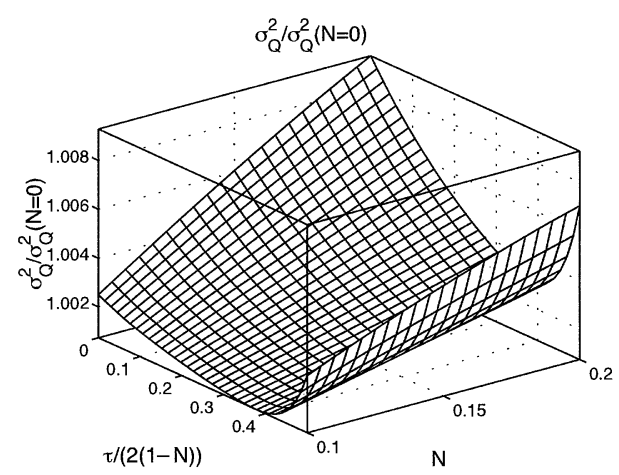

(b)

Figure 11. Intraday patterns of returns under asymmetric information for different lengths of closures. The figures plot the time variation of, Panel A, the instantaneous mean excess share return, $e$, and, Panel $\mathrm{B}$, the return volatility, $\sigma_{Q}$, for different values of the length of closures, $N$. For ease of comparison, the mean return and return volatility are normalized by their respective values in the absence of closures, $e(N=0)$ and $\sigma_{Q}^{2}(N=0)$. Other parameters are set at the following values: $\omega=0.05, \gamma=1000, r=0.001, \sigma_{D}=0.08, a_{G}=0.85, \sigma_{G}=0.08, \sigma_{q}=$ $0.5, a_{Y}=0.25, \sigma_{1}=\sigma_{2}=7, \kappa_{D q}=0.5, \kappa_{12}=0$.

market being permanently open. Our intuition suggests that there is less variation across the trading day in mean return and return volatility. As $N$ gets larger (and $T$ gets smaller), we expect more intraday variation in these quantities, reflecting the increasing importance of market closure. From Figure 11 we see that even for small $N / T$ (around 0.1), the closures can still give rise to U-shaped intraday patterns in mean return and return volatility. For larger values of $N / T$, the mean return and return volatility both become monotonically increasing.

\section{Discrete Simple Returns}

We now reconsider the discrete returns related to some of the empirical results. We use the same parameter values as in Table $I$ for the case of symmetric information (except that $\omega$ is now 0.05 instead of 0.5 ). The first two moments are reported in Table II. The mean return over the trading periods is now positive, and the mean return over the nontrading periods is negative. This is simply due to the fact that the risk discount on the stock $\lambda_{0}$, which depends on the degree of information asymmetry, is higher at the open than at the close (see Figure 5) hence the (mean) stock price is lower at the open than at the close. From the periodicity of the equilibrium, it then follows that the daily return is positive and the nightly return is negative.

The return over trading periods is more volatile than the return over nontrading periods. For the parameter values chosen here, the variance during market open is 0.0166 , which is larger than the variance during market close which is 0.0148 . The ratio between the variance of returns over the 
Table II

\section{Discrete Stock Returns under Asymmetric Information}

This table reports the unconditional mean and variance of various discrete returns produced by the model under asymmetric information. $R_{o o}, R_{c c}, R_{o c}$, and $R_{c o}$ denote, respectively, the simple stock returns from a market open to the next open, from a market close to the next close, from a market open to the next close, and from a market close to the next open. The parameters are set at the following values: $T=0.5, N=0.5, \omega=0.5, \gamma=1000, r=0.001, \sigma_{D}=0.08, a_{G}=0.85$, $\sigma_{G}=0.08, \sigma_{q}=0.5, a_{Y}=0.25, \sigma_{1}=\sigma_{2}=7, \kappa_{D q}=0.5, \kappa_{12}=0$.

\begin{tabular}{lcc}
\hline Returns & Mean $\left(10^{-4}\right)$ & Variance \\
\hline$R_{o o}$ & 0.00 & 0.0454 \\
$R_{c c}$ & 0.00 & 0.0441 \\
$R_{o c}$ & 4.80 & 0.0166 \\
$R_{c o}$ & -4.80 & 0.0148 \\
\hline
\end{tabular}

trading period and the nontrading period is 1.12 . Note that we have chosen the length of trading periods and nontrading periods to be the same and the exogenous information flow to be constant over time. Under symmetric information, the volatility of returns over the trading periods and over the nontrading periods is the same (see Table I). Under asymmetric information, trading among investors reveals investors' private information to the market. Consequently, returns over trading periods are more volatile than returns over nontrading periods since more information is impounded into the equilibrium price over the trading periods. Table II also shows that the return variance from open-to-open, 0.0454 , is larger than the return variance from close-to-close, 0.0441 . The ratio between the variances of the two returns is 1.03 .

\section{Concluding Remarks}

In this paper, we consider a continuous-time stock market with periodic market closures where investors optimally trade for both hedging and speculative reasons. We show that periodic market closures can generate variations in the trading and return distribution that are consistent with the empirical findings. In general, open-to-open returns are more volatile than close-to-close returns and intraday trading volume is U-shaped. When investors have asymmetric information about the stock's future cash flows, (1) the intraday returns and volatility can be U-shaped as well, (2) returns are positive over periods of market open but negative over periods of market close, and (3) returns are more volatile over the trading periods than over the nontrading periods. Our results point to the importance of market closures in explaining documented intraday and intraweek return patterns.

The paper focuses solely on the impact of market closures on trading and returns. Details of the market microstructure concerning the actual trading mechanism are ignored. Also, by assuming a competitive stock market, the 
paper rules out any strategic behavior on the part of investors (see, e.g., Kyle (1985) and Vayanos (1999)). Given that the patterns of interest here are on daily or weekly frequency, these factors can be important in fully understanding the empirical patterns.

We have also made simplifying assumptions on the model for parsimony, but some of these assumptions can be partially relaxed. For example, the model allows only two classes of investors. The better-informed investors (class 1) trade for both hedging and speculative reasons. In general, hedging trades and speculative trades need not come from the same set of investors. We can have hedging and speculative trade initiated from different investors (while still maintaining a hierarchical information structure).

For simple exposition, we have also assumed that all of the informed investors continuously receive new information about the dividend growth of the stock. This assumption can also be relaxed by only allowing a subset of informed investors to receive each piece of private information. This alternative assumption does not affect the equilibrium as long as the informed investors receive the same information on other state variables. In this case, the other informed investors, knowing everything else, can extract that piece of private information from the market clearing price.

Another assumption of the model is continuous dividends from the stock. Although seemingly unrealistic, this assumption is harmless and is made only for ease of exposition. We can assume instead that cumulative dividends are paid out infrequently. In this case, we only need to include the accrued dividend in the stock price. The equilibrium return distributions remain the same and the investors' investment and consumption decisions are unaffected (investors' consumption decisions do not depend on how dividends are paid since they can finance any desired consumption policy for any finite time-period through the money market).

Although the focus of this paper is on the time variation in trading and returns generated by market closures, the model developed here can also be used to analyze other issues and phenomena associated with market closures such as welfare implications of market closures, overnight trading, the interaction across markets in different time zones (see, e.g., Barclay et al. (1990), Chan et al. (1996)), etc. We leave these issues for future research.

\section{Appendix A. Mathematical Preliminaries}

This appendix provides some technical results needed for later use.

We begin by introducing some additional notation. Given a matrix $\boldsymbol{m}, m_{i j}$ denotes its $(i j)$ element, $\boldsymbol{m}_{(i, \cdot)}$ its $i$-th row, $\boldsymbol{m}_{(i, p: q)}$ the row vector with elements in the $i$-th row from the $p$-th to $q$-th column, $\boldsymbol{m}_{(p: q, j)}$ the column vector with elements in the $j$-th column from the $p$-th to $q$-th row, and $\operatorname{tr}(\boldsymbol{m})$ its trace. Additionally, let $[\boldsymbol{m}]$ be the column matrix consisting of its independent elements and $\|\boldsymbol{m}\|=\max \left|m_{i, j}\right|$, its norm. When $\boldsymbol{m}$ is positive semidefinite (positive definite), we state $\boldsymbol{m} \geq 0(>0)$. Also, let $\boldsymbol{i}_{i j}^{(m, n)}$ be an index matrix of order $m \times n$ with its $(i j)$-th element being one and all the other 
elements being zero. And let $\mathbf{1}^{(n)}$ be an identity matrix of rank $n . \Theta=\{r>0$, $\gamma>0, a_{G}>0, a_{Y}>0, \sigma_{D} \geq 0, \sigma_{G} \geq 0, \sigma_{1} \geq 0, \sigma_{2} \geq 0, \sigma_{q} \geq 0,0<\kappa_{D q}<1,-1 \leq$ $\left.\kappa_{12} \leq 1, T \geq 0, N \geq 0\right\}$ denotes the set of parameter values and $\theta \in \Theta$ denotes a generic element. For $d \boldsymbol{X}_{j}=\boldsymbol{a}_{X_{j}} d t+\boldsymbol{b}_{X_{j}} d \boldsymbol{w}_{t}, j=1,2$, let $\boldsymbol{\sigma}_{X_{i} X_{j^{\prime}}}=\boldsymbol{b}_{X_{j}} \boldsymbol{b}_{X_{j^{\prime}}^{\prime}}$ denote the instantaneous covariation of $\boldsymbol{X}_{\mathbf{j}}$ and $\boldsymbol{X}_{j^{\prime}}$ (e.g., see Karatzas and Shreve (1988) for a discussion of covariation processes).

In deriving several results in the paper, we often encounter the two-point boundary-value problem for a (vector) first-order ODE. Here, we give a formal and relatively general definition of the two-point boundary-value problem and state some known results concerning its solution.

Definition A.1: Let $\boldsymbol{f}: \mathfrak{R}_{+} \otimes \mathfrak{R}^{n} \otimes \mathfrak{R}^{m} \otimes \Re^{\prime} \rightarrow \mathfrak{R}^{n}$, and $\boldsymbol{g}: \mathfrak{R}^{n} \otimes \Re^{m} \otimes$ $\mathfrak{R} \rightarrow \mathfrak{R}^{n}$. A two-point boundary-value problem is defined as

$$
\left\{\begin{array}{l}
\dot{\boldsymbol{z}}=\boldsymbol{f}(t, \boldsymbol{z} ; \theta, \omega) \quad \forall t \in[0, T] \\
\mathbf{0}=\boldsymbol{g}[\boldsymbol{z}(0), \boldsymbol{z}(T) ; \theta, \omega]
\end{array}\right.
$$

where $T>0, \theta \in \Theta$, and $\omega \in[0,1]$.

We also define the terminal value problem:

$$
\left\{\begin{array}{l}
\dot{\boldsymbol{z}}=\boldsymbol{f}(t, \boldsymbol{z} ; \theta, \omega) \quad \forall t \in[0, T] \\
\boldsymbol{z}(T)=\boldsymbol{z}_{T} .
\end{array}\right.
$$

Under appropriate smoothness conditions on $\boldsymbol{f}(t, \boldsymbol{z} ; \theta, \omega)$, system (A2) has a unique solution $\boldsymbol{z}=\boldsymbol{z}\left(t ; \theta, \omega ; \boldsymbol{z}_{T}\right)$, which is differentiable in $\boldsymbol{z}_{T}$ (see Keller (1992), Thm. 1.1.1). Solving the two-point boundary-value (A1) is to seek a value for $\boldsymbol{z}_{T}$ that solves

$$
\mathbf{0}=\boldsymbol{g}\left[\boldsymbol{z}\left(0 ; \theta, \omega ; \boldsymbol{z}_{T}\right), \boldsymbol{z}_{T} ; \theta, \omega\right] \equiv \boldsymbol{g} \circ \boldsymbol{z}\left(\boldsymbol{z}_{T} ; \theta, \omega\right) .
$$

The existence of a root to equation (A3) relies on the properties of $\boldsymbol{g} \circ \boldsymbol{z}\left(\boldsymbol{z}_{T} ; \theta, \omega\right)$. Furthermore, let $(\boldsymbol{g} \circ \boldsymbol{z}+1)\left(\boldsymbol{z}_{T} ; \theta, \omega\right) \equiv \boldsymbol{g} \circ \boldsymbol{z}\left(\boldsymbol{z}_{T} ; \theta, \omega\right)+\boldsymbol{z}_{T}$.

Lemma A.1: If $(\boldsymbol{g} \circ \boldsymbol{z}+1)(\cdot ; \theta, \omega): \Re^{n} \rightarrow \Re^{n}$ is continuous and there exists a nonempty, closed, bounded, and convex subset of $\Re^{n}, L$, such that $(\boldsymbol{g} \circ \boldsymbol{z}+1)$ $(\cdot ; \theta, \omega)$ maps $L$ into itself, then equation (A3) has a root and the two-point boundary value problem (A1) has a solution.

Proof: Existence of a root to equation (A3) follows from Brouwer's Fixed Point Theorem (see, e.g., Border (1985, p. 28), Cronin (1994, p. 352)). Q.E.D.

The condition on $(\boldsymbol{g} \circ \boldsymbol{z}+1)$ required by Lemma A.1 is not always easy to verify, in which case the existence of a solution to problem (A1) is not readily confirmed. However, if a solution exists for $\omega_{0}$, the existence of a solution for $\omega$ close to $\omega_{0}$ is easy to establish. 
Definition A.2: $\boldsymbol{z}=\boldsymbol{z}\left(t ; \theta ; \omega_{0}\right)$ is an isolated solution of system (A1) if the linearized system

$$
\left\{\begin{array}{l}
\dot{\boldsymbol{y}}=\nabla_{z} \boldsymbol{f}\left(t, \boldsymbol{z} ; \theta, \omega_{0}\right) \quad \forall t \in[0, T] \\
0=\nabla_{z} \boldsymbol{g}\left(\boldsymbol{z}_{0} ; \theta, \omega_{0}\right) \boldsymbol{y}(0)+\nabla_{z} \boldsymbol{g}\left(\boldsymbol{z}_{T} ; \theta, \omega_{0}\right) \boldsymbol{y}(T)
\end{array}\right.
$$

has $\boldsymbol{y}=0$ as the only solution, where $\nabla$ denotes the partial derivative operator.

Lemma A.2: Suppose that (a) system (A1) has an isolated solution $\boldsymbol{z}=\boldsymbol{z}\left(t ; \theta, \omega_{0}\right)$ for $\omega=\omega_{0}$, and $(\mathrm{b}) \boldsymbol{f}(t, \cdot ; \theta, \omega)$ and $\boldsymbol{g}(\cdot ; \theta, \omega)$ are continuously differentiable in the neighborhood of $\left(t, \boldsymbol{z}\left(t ; \theta, \omega_{0}\right), \omega_{0}\right)$. Then, system (A1) has a solution for $\omega$ close to $\omega_{0}$.

Proof: See Keller (1992, p. 199). Q.E.D.

We next state the general form of the boundary-value problems we encounter in the paper and show that they can be reduced to the two-point boundary-value problem (A1). Let $j=a, b, \bar{T}=T+N$, and $\boldsymbol{z}=\left[\boldsymbol{z}_{a} ; \boldsymbol{z}_{b}\right]$. Our boundary value problem is given by:

$$
\begin{aligned}
\dot{\boldsymbol{z}} & =\boldsymbol{f}(\boldsymbol{z}, \theta, \omega), \quad \forall t \in[0, T] \\
\boldsymbol{z}_{a}(T) & =\boldsymbol{g}_{a}^{c}\left[\boldsymbol{z}_{a}\left(T^{+}\right) ; \theta, \omega\right] \quad \text { and } \quad \boldsymbol{z}_{b}(T)=\boldsymbol{z}_{b}\left(T^{+}\right), \quad t=T \\
\dot{\boldsymbol{z}}_{j} & =\boldsymbol{f}_{j}\left(\boldsymbol{z}_{j}, \theta, \omega\right), \quad \forall t \in(T, \bar{T}) \quad \text { and } j=a, b ; \\
\boldsymbol{z}_{a}\left(\bar{T}^{-}\right) & =\boldsymbol{g}_{a}^{o}\left[\boldsymbol{z}_{a}(\bar{T}) ; \theta, \omega\right] \quad \text { and } \quad \boldsymbol{z}_{b}(\bar{T})=\boldsymbol{g}_{b}^{o}\left[\boldsymbol{z}_{b}\left(\bar{T}^{-}\right) ; \theta, \omega\right], \quad t=\bar{T} \\
\boldsymbol{z}(0) & =\boldsymbol{z}(\bar{T})
\end{aligned}
$$

where $\boldsymbol{f}, \boldsymbol{g}_{j}^{c}, \boldsymbol{f}_{j}$, and $\boldsymbol{g}_{j}^{o}$ are continuously differentiable. Given $\boldsymbol{z}_{\alpha}(0)$, periodicity condition (A5e) and condition (A5d) give $\boldsymbol{z}_{a}\left(\bar{T}^{-}\right)=\boldsymbol{g}_{a}^{o}\left[\boldsymbol{z}_{a}(0) ; \theta, \omega\right]$ as the terminal condition for equation (A5c). Integrating equation (A5c) yields $\boldsymbol{z}_{a}\left(T^{+}\right)=\boldsymbol{z}_{a}^{N}\left(T^{+} ; \boldsymbol{g}_{a}^{o}\left[\boldsymbol{z}_{a}(0) ; \theta, \omega\right]\right)$. Equation (A5b) gives a two-point boundary condition for $\boldsymbol{z}_{a}(0)$ and $\boldsymbol{z}_{\alpha}(T)$ :

$$
\boldsymbol{z}_{a}(T)=\boldsymbol{g}_{a}^{c}\left[\boldsymbol{z}_{a}^{N}\left(T^{+} ; \boldsymbol{g}_{a}^{o}\left[\boldsymbol{z}_{a}(0) ; \theta, \omega\right]\right) ; \theta, \omega\right] \equiv \boldsymbol{g}_{a}^{c} \circ \boldsymbol{z}_{a}^{N} \circ \boldsymbol{g}_{a}^{o}\left(\boldsymbol{z}_{a}(0) ; \theta, \omega\right) .
$$

Given $\boldsymbol{z}_{b}(T)$, conditions (A5b) and (A5c) lead to $\boldsymbol{z}_{b}^{N}\left(\bar{T}^{-} ; \boldsymbol{z}_{b}(T)\right)$. From equation (A5d) it follows that $\boldsymbol{z}_{b}(\bar{T})=\boldsymbol{g}_{b}^{o}\left[\boldsymbol{z}_{b}^{N}\left(\bar{T}^{-} ; \boldsymbol{z}_{b}(T)\right) ; \theta, \omega\right]$. Condition (A5e) gives

$$
\boldsymbol{z}_{b}(0)=\boldsymbol{g}_{b}^{o}\left[\boldsymbol{z}_{b}^{N}\left(\bar{T}^{-} ; \boldsymbol{z}_{b}(T) ; \theta, \omega\right) ; \theta, \omega\right] \equiv \boldsymbol{g}_{b}^{o} \circ \boldsymbol{z}_{b}^{N}\left(\boldsymbol{z}_{b}(T) ; \theta, \omega\right)
$$


Our boundary-value problem now reduces to the system given by equations (A5a), (A6), and (A7), which is in the form of problem (A1).

For future use, we also state two auxiliary lemmas, which are needed in proving Lemma 1, Lemma 3, and Theorem 1.

Definition A.3: Let $\boldsymbol{a}_{0} \geq 0$ and $\boldsymbol{a}_{2}>0$ be constant, symmetric matrices. Also let $\boldsymbol{r}$ be the variable of interest, which is a symmetric matrix. Finally, let $\boldsymbol{a}_{3}(t, \boldsymbol{r})$ be a positive, linear operator mapping symmetric matrices into themselves. A matrix Riccati differential equation is defined as

$$
\dot{\boldsymbol{r}}+\boldsymbol{a}_{0}+\left(\boldsymbol{a}_{1}^{\prime} \boldsymbol{r}+\boldsymbol{r} \boldsymbol{a}_{1}\right)-\boldsymbol{r a} \boldsymbol{a}_{2} \boldsymbol{r}+\boldsymbol{a}_{3}(t, \boldsymbol{r})=0 \quad \text { (a.e.) } \quad \forall t \in[0, T],
$$

where $\dot{\boldsymbol{r}}=d \boldsymbol{r} / d t$ and $\boldsymbol{r}(T)=\boldsymbol{r}_{T}$.

Lemma A.3: For any given terminal value $\boldsymbol{r}_{T} \geq 0$, the matrix Riccati equation (A8) has a unique, symmetric, positive, semidefinite solution. Let $\boldsymbol{m}$ be an arbitrary (bounded measurable) matrix defined on $[0, T]$ and let $\boldsymbol{e}(t)$ be the solution of the following linear equation:

$$
\dot{\boldsymbol{e}}+\boldsymbol{a}_{0}+\left(\boldsymbol{a}_{1}-\boldsymbol{m}\right)^{\prime} \boldsymbol{e}+\boldsymbol{e}\left(\boldsymbol{a}_{1}-\boldsymbol{m}\right)+\boldsymbol{m}^{\prime} \boldsymbol{a}_{2} \boldsymbol{m}+\boldsymbol{a}_{3}(t, \boldsymbol{e})=0 \quad \forall t \in[0, T],
$$

where $\boldsymbol{e}(T)=\boldsymbol{r}_{T}$. If $\boldsymbol{r}(t)$ is the solution of equation (A8), then $\boldsymbol{r}(t) \leq \boldsymbol{e}(t), \forall t \in$ $[0, T]$.

Proof: See Wonham (1968). Q.E.D.

Lemma A.4: Let $f: \mathcal{D} \rightarrow \Re$ be an analytic function, where $\mathcal{D}=D_{1} \otimes \cdots \otimes D_{n}$ is an open, bounded subset of $\Re^{n}$. Let $\mathcal{Z}=\{x \in \mathcal{D}: f(x)=0\}$ be its zero set. Then, either $\mathcal{Z}=\mathcal{D}$ or $\mu_{n}(\mathcal{Z})=0$, where $\mu_{n}$ is the $n$-dimensional Lebesgue measure.

Proof: We prove Lemma A.4 by induction. First note that $\mathcal{Z}$ is closed and therefore measurable. For $n=1, \mathcal{Z}$ is either finite or has an accumulation point. In the latter case, $f$ is identically zero on $\mathcal{D}$ (see, e.g., Ahlfors (1979)). Noting that any finite set has zero Lebesgue measure concludes this part of the proof. Let us suppose that the conclusion of the lemma holds for certain $k \geq 1$ and prove it for $n=k+1$. We can write $f$ as a function of two variables $f(t, x)$ on $\mathcal{D}_{1} \otimes \mathcal{D}_{2}$, where $\mathcal{D}_{2}=D_{2} \otimes \cdots \otimes D_{k+1}$. Clearly, $f$ is a real analytic function in both $t$ and $x$ separately. Consider the set $\mathcal{S}=$ $\left\{t \in \mathcal{D}_{1}: \forall x \in \mathcal{D}_{2}, f(t, x)=0\right\}$. For $t \notin \mathcal{S}, \int_{\mathcal{D}_{2}} 1_{f(t, x)=0} d x=0$ by the inductive assumption. If $\mathcal{S}$ is finite, it is of zero Lebesgue measure in $\mathcal{D}_{1}$. Thus, $\mu_{n}(\mathcal{Z})=\int_{\mathcal{D}_{1}} \int_{\mathcal{D}_{2}} 1_{f(t, x)=0} d x d t=0$ by the Fubini theorem (see, e.g., Doob (1991)). If $\mathcal{S}$ is not finite, it has an accumulation point. From the result for $n=1, \forall x \in \mathcal{D}_{2}, f(t, x)$ is identically zero in $\mathcal{D}_{1}$. Thus, $f(t, x)$ is identically zero on $\mathcal{D}=\mathcal{D}_{1} \otimes \mathcal{D}_{2}$. Q.E.D. 


\section{Appendix B. Proofs of Lemmas 1-3}

Proof of Lemma 1: We first derive the results for a general system and then show that our system is a special case of the general system. Consider the following general system. Let $\boldsymbol{Z}_{t}$ be the state vector, where $\boldsymbol{Z}_{t}$ follows:

$$
\boldsymbol{Z}_{t}=\boldsymbol{Z}_{0}+\int_{0}^{t}\left(-\boldsymbol{a}_{Z} \boldsymbol{Z}_{s} d s+\boldsymbol{b}_{Z} d \boldsymbol{w}_{s}\right)
$$

where $\boldsymbol{a}_{Z}(>0)$ and $\boldsymbol{b}_{Z}$ are constant over time. Next, define

$$
\begin{aligned}
& \boldsymbol{S}_{t}^{a}=\boldsymbol{S}_{0}^{a}+\int_{0}^{t}\left(\boldsymbol{a}_{S^{a}} \boldsymbol{Z}_{s} d s+\boldsymbol{b}_{S^{a}} d \boldsymbol{w}_{s}\right), \\
& \boldsymbol{S}_{t}^{b}=\boldsymbol{S}_{0}^{b}+\int_{0}^{t}\left(\boldsymbol{a}_{S^{b}} \boldsymbol{Z}_{s} d s+\boldsymbol{b}_{S^{b}} d \boldsymbol{w}_{s}\right),
\end{aligned}
$$

where $\boldsymbol{a}_{S^{a}}, \boldsymbol{b}_{S^{a}}, \boldsymbol{a}_{S^{b}}$, and $\boldsymbol{b}_{S^{b}}$ are deterministic and bounded. Then we define $\boldsymbol{S}_{\mathrm{t}}=\left[\boldsymbol{S}_{t}^{a} ; \boldsymbol{S}_{t}^{b}\right]$ to be the signal vector during the day and $\boldsymbol{S}_{t}^{*}=\boldsymbol{S}_{t}^{b}$ to be the signal vector during the night. Then

$$
\begin{aligned}
& t \in \mathcal{T}: \quad \boldsymbol{S}_{t}=\boldsymbol{S}_{0}+\int_{0}^{t}\left(\boldsymbol{a}_{S} \boldsymbol{Z}_{s} d s+\boldsymbol{b}_{S} d \boldsymbol{w}_{s}\right), \\
& t \in \mathcal{N}: \quad \boldsymbol{S}_{t}^{*}=\boldsymbol{S}_{0}^{*}+\int_{0}^{t}\left(\boldsymbol{a}_{S^{*}} \boldsymbol{Z}_{s} d s+\boldsymbol{b}_{S^{*}} d \boldsymbol{w}_{s}\right),
\end{aligned}
$$

where $\boldsymbol{a}_{S}, \boldsymbol{b}_{S}, \boldsymbol{a}_{S^{*}}$, and $\boldsymbol{b}_{S^{*}}$ are deterministic and bounded. Further assume that $\boldsymbol{b}_{S} \boldsymbol{b}_{S}^{\prime}$ and $\boldsymbol{b}_{S^{*}} \boldsymbol{b}_{S^{*}}^{\prime}$ are uniformly nonsingular on $\mathcal{T}$ and $\mathcal{N}$ respectively. Additionally, $\boldsymbol{Z}_{0}$ and $\boldsymbol{S}_{0}$ are jointly normal. Let $\mathcal{H}_{t}$ be observations of $\boldsymbol{S}$ and $\boldsymbol{S}^{*}$ up to $t$ (including the prior).

The first part of the lemma states that the process $\left\{\boldsymbol{Z}_{t},\left(\boldsymbol{S}_{t}, \boldsymbol{S}_{t}^{*}\right)\right\}$ is conditionally Gaussian; that is, $\forall s, s_{1}, \ldots, s_{n} \in[0, t](t \geq 0),\left\{\boldsymbol{Z}_{s_{1}}, \ldots, \boldsymbol{Z}_{s_{n}}\right\}$ has a joint Gaussian distribution conditioned on $\mathcal{H}_{s}$. We prove this by induction: We first show that the result holds for $t<t_{1}$, and then show that it also holds for $t<t_{k+1}$ if it holds for $t<t_{k}$. Since $\boldsymbol{Z}_{0}$ and $\boldsymbol{S}_{0}$ are jointly normal, it follows that $\boldsymbol{Z}_{0}$ conditioned on $\boldsymbol{S}_{0}$ is Gaussian. For $t<n_{0}$, our result follows as a special case of Theorem 12.6 of Liptser and Shiryayev (1978). Since $\boldsymbol{Z}_{n_{0}}$ conditioned on $\mathcal{H}_{n_{0}}$ is Gaussian and $\boldsymbol{a}_{S^{*}}$ and $\boldsymbol{b}_{S^{*}}$ are bounded, the result continues to hold for $t<t_{1}$. Suppose the result holds for $t<t_{k}$ with $k>1$. Since $\boldsymbol{Z}_{t_{k}}$ conditioned on $\mathcal{H}_{t_{\bar{k}}}$ is Gaussian, by the same argument as for 
$t_{0}=0, \boldsymbol{Z}_{t_{k}}$ conditioned on $\mathcal{H}_{t_{k}}$ is also Gaussian. By Theorem 12.6 of Liptser and Shiryayev again, the result also holds for $t<t_{k+1}$. The result then follows.

Let $\hat{\boldsymbol{Z}}_{t}=\mathbf{E}\left[\boldsymbol{Z}_{t} \mid \mathcal{H}_{t}\right]$ and $\boldsymbol{o}(t)=\mathrm{E}\left[\left(\boldsymbol{Z}_{t}-\hat{\boldsymbol{Z}}_{t}\right)\left(\boldsymbol{Z}_{t}-\hat{\boldsymbol{Z}}_{t}\right)^{\prime} \mid \mathcal{H}_{t}\right]$. We now prove the second part of the lemma. For $t \in \mathcal{T}_{k}$ or $t \in \mathcal{N}_{k}$, the dynamics of $\hat{Z}_{t}$ and $\boldsymbol{o}(t)$ follow from Theorem 10.3 of Liptser and Shiryayev (1977). At $t=n_{k}$, both $\hat{Z}$ and $\boldsymbol{o}$ are continuous. At $t=t_{k}$, given $\hat{Z}_{t_{\bar{k}}^{-}}$and $\boldsymbol{o}\left(t_{k}^{-}\right)$, we can obtain $\hat{Z}_{t_{k}}$ and $\boldsymbol{o}\left(t_{k}\right)$ from the fact that $\boldsymbol{S}_{t_{k}}^{a}$ can be written as $\boldsymbol{S}_{t_{k}}^{a}=\tilde{\boldsymbol{\lambda}}_{Z}\left(t_{k}\right) \boldsymbol{Z}_{t_{k}}+\boldsymbol{b}_{\tilde{\lambda}}\left(t_{k}\right) \boldsymbol{\epsilon}_{t_{k}}$ (where $\boldsymbol{\epsilon}_{t_{k}}$ is a standard normal vector) and the law of conditional expectations for Gaussian variables (see, e.g., Jazwinski (1970, Thm. 7.1)). Summarizing these results, we have

$$
\begin{aligned}
t=t_{k}: & \hat{Z}_{t_{k}}=\hat{Z}_{t_{k}^{-}}+\boldsymbol{k}^{o}\left(\boldsymbol{S}_{t_{k}}^{a}-\mathrm{E}\left[\boldsymbol{S}_{t_{k}}^{a} \mid \mathcal{H}_{t}\right]\right) ; \quad \boldsymbol{o}\left(t_{k}\right)=\boldsymbol{o}\left(t_{k}^{-}\right)+\boldsymbol{g}_{o}^{o} \\
t \in\left[t_{k}, n_{k}\right]: & d \hat{Z}_{t}=\boldsymbol{a}_{Z} \hat{Z}_{t} d t+\boldsymbol{k}\left(d \boldsymbol{S}_{t}-\mathrm{E}\left[d \boldsymbol{S}_{t} \mid \mathcal{H}_{t}\right]\right) ; \quad \dot{\boldsymbol{o}}=\boldsymbol{g}_{o} ; \\
t=n_{k}: & \hat{Z}_{n_{k}^{+}}=\hat{Z}_{n_{k}} ; \quad \boldsymbol{o}\left(n_{k}\right)=\boldsymbol{o}\left(n_{k}^{+}\right)+\boldsymbol{g}_{o}^{c} ; \\
t \in\left(n_{k}, t_{k+1}\right): & d \hat{Z}_{t}=\boldsymbol{a}_{Z} \hat{Z}_{t} d t+\boldsymbol{k}^{*}\left(d \boldsymbol{S}_{t}^{*}-\mathrm{E}\left[d \boldsymbol{S}_{t}^{*} \mid \mathcal{H}_{t}\right]\right) ; \quad \dot{\boldsymbol{o}}=\boldsymbol{g}_{o}^{*},
\end{aligned}
$$

where $k=0,1,2, \ldots$ and

$$
\begin{aligned}
t=t_{k}: \quad \boldsymbol{k}^{o}=\boldsymbol{o}\left(t_{k}^{-}\right) \tilde{\boldsymbol{\lambda}}_{Z}^{\prime}\left(t_{k}\right)\left[\tilde{\boldsymbol{\lambda}}_{Z}\left(t_{k}\right) \boldsymbol{o}\left(t_{k}^{-}\right) \tilde{\boldsymbol{\lambda}}_{Z}^{\prime}\left(t_{k}\right)+\boldsymbol{b}_{\tilde{\lambda}} \boldsymbol{b}_{\tilde{\lambda}}^{\prime}\right]^{-1} ; \\
\boldsymbol{g}_{o}^{o}=-\boldsymbol{k}^{o} \tilde{\boldsymbol{\lambda}}_{Z}\left(t_{k}\right) \boldsymbol{o}\left(t_{k}^{-}\right) ; \\
t \in\left[t_{k}, n_{k}\right]: \quad \boldsymbol{k}=\left(\boldsymbol{\sigma}_{Z S}+\boldsymbol{o} \boldsymbol{a}_{S}^{\prime}\right) \boldsymbol{\sigma}_{S S}^{-1} ; \\
\boldsymbol{g}_{o}=-\left(\boldsymbol{a}_{Z} \boldsymbol{o}+\boldsymbol{o} \boldsymbol{a}_{Z}^{\prime}\right)+\boldsymbol{\sigma}_{Z Z}-\boldsymbol{k}\left(\boldsymbol{\sigma}_{Z S}^{\prime}+\boldsymbol{a}_{S} \boldsymbol{o}\right) ; \\
t=n_{k}: \quad \boldsymbol{k}^{c}=\boldsymbol{k}^{*}\left(n_{k}^{+}\right) ; \\
\boldsymbol{g}_{o}^{c}=\mathbf{0} ; \\
t \in\left(n_{k}, t_{k+1}\right): \quad \boldsymbol{k}^{*}=\left(\boldsymbol{\sigma}_{Z S^{*}}+\boldsymbol{o a}_{S *}^{\prime}\right) \boldsymbol{\sigma}_{S^{*} S^{*}}^{-1} ; \\
\boldsymbol{g}_{o}^{*}=-\left(\boldsymbol{a}_{Z} \boldsymbol{o}+\boldsymbol{o a}_{Z}^{\prime}\right)+\boldsymbol{\sigma}_{Z Z}-\boldsymbol{k}^{*}\left(\boldsymbol{\sigma}_{Z S^{*}}^{\prime}+\boldsymbol{a}_{S^{*}} \boldsymbol{o}\right) .
\end{aligned}
$$

In particular, $\boldsymbol{o}$ is deterministic and independent of $\mathcal{H}_{t}$. It only depends on the parameters of the system (e.g., $\boldsymbol{a}_{Z}, \boldsymbol{b}_{Z}, \boldsymbol{a}_{S}, \boldsymbol{b}_{S}, \boldsymbol{a}_{S^{*}}$, and $\boldsymbol{b}_{S^{*}}$ ) and the initial condition.

The problem we consider in the paper maps directly into the system of equations (B1)-(B3). First, note that our state vector given in equation (3) maps over to equation (B1) since $\boldsymbol{a}_{\mathrm{Z}}=\operatorname{diag}\left\{a_{G}, a_{Y}, a_{Y}\right\}>0$ and $\boldsymbol{b}_{Z}=\left[\boldsymbol{b}_{G} ; \boldsymbol{b}_{1} ; \boldsymbol{b}_{2}\right]$ are con- 
stant through time. Second, we show that the investors' signal vectors $\boldsymbol{S}_{i, t}$ can be mapped into system (B1)-(B3). To begin with, observe that these signal vectors can be rewritten in a more compact form. For investor 1 who observes all the state variables, $P_{t}$ is a redundant signal. For investor 2 , observing $P_{t}$ is equivalent to observing $\boldsymbol{\lambda}_{1} \mathrm{E}_{1, t}\left[\boldsymbol{Z}_{t}\right]=\boldsymbol{\lambda}_{1}\left[G_{t}, Y_{1, t}, Y_{2, t}\right]^{\prime}$. Knowing $Y_{2, t}$, observing $\lambda_{1} \mathrm{E}_{1, t}\left[\boldsymbol{Z}_{t}\right]$ allows him to infer $\lambda_{1 G} G_{t}-\lambda_{11} Y_{1, t}$. Define $\tilde{P}_{i, t}=\tilde{\boldsymbol{\lambda}}_{i} \boldsymbol{Z}_{t}$ where $\tilde{\boldsymbol{\lambda}}_{1}=$ $[0,0,1]$ and $\tilde{\lambda}_{2}=\left[\lambda_{1 G},-\lambda_{11}, 0\right]$ for $t \in \mathcal{T}$ and $\tilde{\lambda}_{1}=\tilde{\lambda}_{2}=0$ for $t \in \mathcal{N}$. Hence $\widetilde{P}_{i, t}$ denotes the effective signal investor $i$ extracts from the price. Combined with the other signals, investor $i$ 's signal vector $\boldsymbol{S}_{i, t}$ can be rewritten as: $\boldsymbol{S}_{1, t}=\left[\boldsymbol{U}_{t} ; D_{t} ; \widetilde{P}_{1, t} ; G_{t} ; Y_{1, t} ; Y_{2, t} ; q_{1, t}\right]$ and $\boldsymbol{S}_{2, t}=\left[\boldsymbol{U}_{t} ; D_{t} ; \tilde{P}_{2, t} ; Y_{2, t} ; q_{2, t}\right]$. For investor $1, \tilde{\boldsymbol{\lambda}}_{Z}=\tilde{\boldsymbol{\lambda}}_{1}, \boldsymbol{b}_{\tilde{\lambda}}=0$ and

$$
\begin{aligned}
\boldsymbol{a}_{S} & =\left[\boldsymbol{a}_{U} ;[1,0,0] ; \dot{\tilde{\boldsymbol{\lambda}}}_{1}-\tilde{\boldsymbol{\lambda}}_{1} \boldsymbol{a}_{Z} ;-\boldsymbol{a}_{Z} ;[0,1,0]\right] ; \quad \boldsymbol{b}_{S}=\left[\boldsymbol{b}_{U} ; \boldsymbol{b}_{D} ; \tilde{\boldsymbol{\lambda}}_{1} \boldsymbol{b}_{Z} ; \boldsymbol{b}_{Z} ; \boldsymbol{b}_{q}\right] ; \\
\boldsymbol{a}_{S^{*}} & =\left[\boldsymbol{a}_{U} ;[1,0,0] ;-\boldsymbol{a}_{Z} ;[0,1,0]\right] ; \quad \boldsymbol{b}_{S^{*}}=\left[\boldsymbol{b}_{U} ; \boldsymbol{b}_{D} ; \boldsymbol{b}_{Z} ; \boldsymbol{b}_{q}\right] .
\end{aligned}
$$

For investor 2 , let $\tilde{\boldsymbol{\lambda}}_{Z}=\tilde{\boldsymbol{\lambda}}_{2}, \boldsymbol{b}_{\tilde{\lambda}}=0$ and

$$
\begin{aligned}
\boldsymbol{a}_{S} & =\left[\boldsymbol{a}_{U} ;[1,0,0] ; \dot{\tilde{\lambda}}_{2}-\tilde{\boldsymbol{\lambda}}_{2} \boldsymbol{a}_{Z} ;\left[0,0,-a_{Y}\right] ;[0,0,1]\right] \\
\boldsymbol{b}_{S} & =\left[\boldsymbol{b}_{U} ; \boldsymbol{b}_{D} ; \tilde{\boldsymbol{\lambda}}_{2} \boldsymbol{b}_{Z} ; \boldsymbol{b}_{2} ; \boldsymbol{b}_{q}\right] ; \\
\boldsymbol{a}_{S^{*}} & =\left[\boldsymbol{a}_{U} ;[1,0,0] ;\left[0,0,-a_{Y}\right] ;[0,0,1]\right] ; \quad \boldsymbol{b}_{S^{*}}=\left[\boldsymbol{b}_{U} ; \boldsymbol{b}_{D} ; \boldsymbol{b}_{2} ; \boldsymbol{b}_{q}\right] .
\end{aligned}
$$

Their signal vectors have the same form as in system (B3). Thus, their conditional expectations are given by system (B4) with appropriate substitutions for $\tilde{\boldsymbol{\lambda}}_{Z}, \boldsymbol{b}_{\tilde{\lambda}}, \boldsymbol{a}_{S}, \boldsymbol{b}_{S}, \boldsymbol{a}_{S^{*}}$, and $\boldsymbol{b}_{S^{*}}$. If we define

$$
\begin{aligned}
t=t_{k}: \quad \boldsymbol{a}_{i, S}=\delta_{D}\left(t_{k}\right) \boldsymbol{a}_{S}\left(t_{k}\right) ; \quad \boldsymbol{b}_{i, S}=\boldsymbol{b}_{S}\left(t_{k}\right) ; \quad \boldsymbol{k}_{i}=\boldsymbol{k}^{o} ; \\
\boldsymbol{g}_{i, o}=\delta_{D}\left(t_{k}\right) \boldsymbol{g}_{o}^{o} ; \\
t \in\left(t_{k}, n_{k}\right): \quad \boldsymbol{a}_{i, S}=\boldsymbol{a}_{S} ; \quad \boldsymbol{b}_{i, S}=\boldsymbol{b}_{S} ; \quad \boldsymbol{k}_{i}=\boldsymbol{k} ; \quad \boldsymbol{g}_{i, o}=\boldsymbol{g}_{o} ; \\
t=n_{k}: \quad \boldsymbol{a}_{i, S}=\boldsymbol{a}_{S^{*}}\left(n_{k}^{+}\right) ; \quad \boldsymbol{b}_{i, S}=\boldsymbol{b}_{S^{*}}\left(n_{k}^{+}\right) ; \quad \boldsymbol{k}_{i}=\boldsymbol{k}^{c} ; \quad \boldsymbol{g}_{i, o}=\boldsymbol{g}_{o}^{c} ; \\
t \in\left(n_{k}, t_{k+1}\right): \quad \boldsymbol{a}_{i, S}=\boldsymbol{a}_{S^{*}} ; \quad \boldsymbol{b}_{i, S}=\boldsymbol{b}_{S^{*}} ; \quad \boldsymbol{k}_{i}=\boldsymbol{k}^{*} ; \quad \boldsymbol{g}_{i, o}=\boldsymbol{g}_{o}^{*} ;
\end{aligned}
$$

where $\delta_{D}\left(t_{k}\right) \equiv \delta_{D}\left(t-t_{k}\right)$ is the Dirac delta-function (see, e.g., Lighthill (1958)), we can then formally express our signal vector $\boldsymbol{S}_{i, t}$ in the following form:

$$
\boldsymbol{S}_{i, t}=\boldsymbol{S}_{i, 0}+\int_{0}^{t}\left(\boldsymbol{a}_{i, S} \boldsymbol{Z}_{s} d s+\boldsymbol{b}_{i, S} d \boldsymbol{w}_{s}\right) \quad(i=1,2),
$$


and $\boldsymbol{Z}_{i, t}$ and $\boldsymbol{o}_{i}(t)$ can be expressed in the form of system given by equations $(16 \mathrm{a})$ and (16b). Given an initial value $\boldsymbol{o}_{i}(s)$, we can find $\boldsymbol{o}_{i}(t)$ by integrating equation (16b).

A periodic solution is given by the initial value $\boldsymbol{o}_{i}\left(t_{k}\right)$, which equals $\boldsymbol{o}_{i}\left(t_{k+1}\right)$. We now prove its existence under the assumption that $\boldsymbol{a}_{S}, \boldsymbol{b}_{S}$, and $\boldsymbol{a}_{S^{*}}, \boldsymbol{b}_{S^{*}}$ are periodic on $\mathcal{T}$ and $\mathcal{N}$, respectively. The following lemma summarizes the result.

Lemma B.1: The system (16b) and (17) has a solution.

Proof: The system (16b) and (17) is equivalent to a special case of the two-point boundary value problem specified in system (A5) with $\boldsymbol{z}=\boldsymbol{z}_{b}=\boldsymbol{o}_{i}$ and $\boldsymbol{f}, \boldsymbol{f}_{b}, \boldsymbol{g}_{b}^{o}$ derived from $\boldsymbol{g}_{\mathrm{i}, \mathrm{o}}$ for $i=1,2$ in an obvious fashion. Hence, proving existence of a periodic solution is equivalent to proving existence of a solution to the analogous two-point boundary value problem. First, we prove a simple property this system satisfies. Using the notation of Lemma A.3, let $\boldsymbol{r}=\boldsymbol{o}_{i}, \boldsymbol{a}_{0}=\boldsymbol{\sigma}_{Z Z}-\boldsymbol{\sigma}_{i, Z S} \boldsymbol{\sigma}_{i, S S}^{-1} \boldsymbol{\sigma}_{i, Z S}^{\prime}, \boldsymbol{a}_{1}=-\boldsymbol{a}_{Z}-\boldsymbol{a}_{i, S}^{\prime} \boldsymbol{\sigma}_{i, S S}^{-1} \boldsymbol{\sigma}_{i, Z S}^{\prime}, \boldsymbol{a}_{2}=$ $\boldsymbol{a}_{i, S}^{\prime} \boldsymbol{\sigma}_{i, S S}^{-1} \boldsymbol{a}_{i, S}$, and $\boldsymbol{a}_{3}(t, \boldsymbol{r})=0$. It is easy to verify that $\boldsymbol{a}_{0}$ and $\boldsymbol{a}_{2}$ are positive definite. Thus, equation (16b) is a matrix Riccati equation. The initial value problem for $\boldsymbol{o}_{i}\left(t_{k}\right)=\boldsymbol{o}_{i, t_{k}}$ has a solution $\boldsymbol{o}_{i}\left(t ; \boldsymbol{o}_{i, t_{k}}\right)$ for $t \in\left[t_{k}, t_{k+1}\right)$. A linear bound on the solution $\left\|\boldsymbol{o}_{i}\left(t ; \boldsymbol{o}_{i, t_{k}}\right)\right\| \leq \alpha_{i}\left\|\boldsymbol{o}_{i, t_{k}}\right\|+\beta_{i}$ can be achieved by letting

$$
\begin{aligned}
& \alpha_{i}=\exp \left\{-\left(2 a_{Y}+\frac{1}{2} T\right)\right\} ; \quad \boldsymbol{\zeta}_{i}=\exp \left\{\left[-\left(\boldsymbol{a}_{Z}+\frac{1}{2} \mathbf{1}^{(3)}\right)\right](T-s)\right\} ; \\
& \beta_{i}=\left\|\int_{0}^{T} \zeta_{i}\left\{\boldsymbol{m}(T-s)^{\prime} \boldsymbol{a}_{2}^{-1} \boldsymbol{m}(T-s)+\boldsymbol{a}_{3}(T-s)+\boldsymbol{a}_{0}(T-s)\right\} \zeta_{i} d s\right\| ;
\end{aligned}
$$

where $\boldsymbol{m}=-\boldsymbol{a}_{i, S}^{\prime} \boldsymbol{\sigma}_{i, S S}^{-1} \boldsymbol{\sigma}_{i, Z S}^{\prime} \oplus \frac{1}{2}$. (Here, $\boldsymbol{m} \oplus \frac{1}{2}$ means adding $\frac{1}{2}$ to each element of matrix $\boldsymbol{m}$.) Clearly, $\alpha_{i}<1$. Next, using the notation of system (A5), define

$$
\begin{aligned}
\boldsymbol{k}_{b}^{o} & =\boldsymbol{o}_{i}\left(t_{k+1}^{-}\right) \tilde{\boldsymbol{\lambda}}_{i}^{\prime}\left(t_{k+1}\right)\left[\tilde{\boldsymbol{\lambda}}_{i}\left(t_{k+1}\right) \boldsymbol{o}_{i}\left(t_{k+1}^{-}\right) \tilde{\boldsymbol{\lambda}}_{i}^{\prime}\left(t_{k+1}\right)\right]^{-1} \\
\boldsymbol{g}_{b}^{o}\left[\boldsymbol{o}_{i}\left(t_{k+1}^{-}\right)\right] & =\boldsymbol{o}_{i}\left(t_{k+1}^{-}\right)-\boldsymbol{k}_{b}^{o} \tilde{\boldsymbol{\lambda}}_{i}\left(t_{k+1}\right) \boldsymbol{o}_{i}\left(t_{k+1}^{-}\right) .
\end{aligned}
$$

Imposing the periodicity condition $\boldsymbol{o}_{i}\left(t_{k+1}\right)=\boldsymbol{o}_{i, t_{k}}$, we can rewrite as $\boldsymbol{o}_{i, t_{k}}=$ $\boldsymbol{g}_{b}^{o}\left[\boldsymbol{o}_{i}\left(t_{k+1}^{-} ; \boldsymbol{o}_{i, t_{k}}\right)\right]$. Let $L_{i}$ be the space of symmetric, positive semidefinite matrices such that their norms are less than $\psi_{i}$ where $\psi_{i}=\beta_{i} /\left(1-\alpha_{i}\right)$. It is clear that $L_{i}$ is a nonempty, closed, bounded convex subset of a finite-dimensional normed vector space. Suppose $\boldsymbol{o}_{i, t_{k}} \in L_{i}$. Let $M_{i}\left(\boldsymbol{o}_{i, t_{k}}\right)=\boldsymbol{g}_{b}^{o}\left[\boldsymbol{o}_{i}\left(t_{k+1}^{-} ; \boldsymbol{o}_{i, t_{k}}\right)\right] . M_{i}$ is clearly continuous and $M_{i}\left(\boldsymbol{o}_{i, t_{k}}\right)$ is symmetric and positive semidefinite. Furthermore, $\left\|M_{i}\left(\boldsymbol{o}_{i, t_{k}}\right)\right\| \leq \beta_{i} /\left(1-\alpha_{i}\right)$. So $M_{i}: L_{i} \rightarrow L_{i}$. By Lemma A.1 the result follows.

This completes the proof of Lemma 1. Q.E.D. 
Proof of Lemma 2: For $t \in \mathcal{T}_{k}, \boldsymbol{\Psi}_{1, t}=\boldsymbol{\Psi}_{t}=\left[\boldsymbol{Z}_{1, t} ; \boldsymbol{Z}_{2, t}\right]$ and $\boldsymbol{\Psi}_{2, t}=\left[\boldsymbol{Z}_{2, t} ; \boldsymbol{Z}_{2, t}\right]$. Next $\boldsymbol{\Psi}_{1, t}=\left[\boldsymbol{Z}_{1, t} ;\left(\boldsymbol{Z}_{2, t}\right)_{(1: 2,1)} ;\left(\boldsymbol{Z}_{1, t}\right)_{(3,1)}\right]$ and $\boldsymbol{\Psi}_{2, t}=\left[\left(\boldsymbol{Z}_{2, t}\right)_{(1: 2,1)} ;\left(\boldsymbol{Z}_{1, t}\right)_{(3,1)} ; \boldsymbol{Z}_{2, t}\right]$ for $t \in \mathcal{N}_{k}$. It is obvious that $\boldsymbol{\Psi}_{i, t}$ is a subvector of $\boldsymbol{\Psi}_{t}$. From the solution of $\boldsymbol{Z}_{i, t}$, it clearly follows a Gaussian Markov process under $\mathcal{I}_{i, t}$. It is immediate that $\Psi_{2, t}$ follows a Gaussian Markov process under $\mathcal{I}_{2, t}$. However, $\left(\boldsymbol{Z}_{2, t}\right)_{(1: 2,1)}$ is Gaussian but not Markovian under $\mathcal{I}_{1, t}$. What remains to be shown is that $\left[\boldsymbol{Z}_{1, t} ;\left(\boldsymbol{Z}_{2, t}\right)_{(1: 2,1)}\right]$ is Markovian under $\mathcal{I}_{1, t}$. Given $\boldsymbol{S}_{2, t}$, this is easy to verify. Thus, $\boldsymbol{\Psi}_{i, t}$ follows a Gaussian Markov process under $\mathcal{I}_{i, t}, i=1,2$. By similar arguments, $\Psi_{t}$ is a Gaussian Markov process under $\mathcal{I}_{t}$. Q.E.D.

Proof of Lemma 3: For the solution to the investors' optimization problem, we derive the evolution of each investor's state vector, $\boldsymbol{X}_{i, t}=\mathbf{E}_{i, t}\left[\boldsymbol{X}_{t}\right]$, given in equation (19). Let $\boldsymbol{m}_{1}=\left[\boldsymbol{i}_{15}^{(1,6)} ; \boldsymbol{i}_{13}^{(1,6)} ; \boldsymbol{i}_{14}^{(1,6)} ;\left[\left(\boldsymbol{\lambda}_{2, Z}\right)_{(1,1)},\left(\boldsymbol{\lambda}_{2, Z}\right)_{(1,2)}, 0,0,0,0\right]\right.$; $\left.\boldsymbol{i}_{16}^{(1,6)}\right]$ and $\boldsymbol{m}_{2}=\boldsymbol{k}_{2} \boldsymbol{a}_{2, \mathrm{~S}}$. Then,

$$
\begin{aligned}
& \boldsymbol{a}_{1, X}=\operatorname{diag}\left\{\operatorname{diag}\left\{0, a_{Y}, a_{Y}\right\}, \operatorname{diag}\left\{a_{G}, a_{Y}\right\}-\boldsymbol{m}_{2,(1: 2,1: 2)}\right\} \\
& \boldsymbol{k}_{1, X}=\left[\mathbf{0} ; \boldsymbol{k}_{1,(2, \cdot)} ; \boldsymbol{k}_{1,(3, \cdot)} ; \boldsymbol{k}_{1,(1: 2, \cdot)}-\boldsymbol{k}_{2,(1: 2, \cdot)} \boldsymbol{m}_{1}\right] \\
& \boldsymbol{a}_{2, X}=\operatorname{diag}\left\{0, a_{Y}, a_{Y}\right\} ; \quad \boldsymbol{k}_{2, X}=\left[\mathbf{0} ; \boldsymbol{k}_{2,(2: 3, \cdot)}\right] .
\end{aligned}
$$

Equation (19) follows immediately from equation (16a). Given the processes of the stock price and the state vector, the return processes for investor $i$ can be expressed as follows:

$$
\begin{aligned}
& d Q_{t}=\boldsymbol{a}_{i, Q} \boldsymbol{X}_{i, t} d t+\boldsymbol{b}_{i, Q} d \boldsymbol{w}_{i, t} \quad \forall t \in \mathcal{T} ; \\
& d q_{i, t}=\boldsymbol{a}_{i, q} \boldsymbol{X}_{i, t} d t+\boldsymbol{b}_{i, q} d \boldsymbol{w}_{i, t} \quad \forall t \in \mathcal{T} \cup \mathcal{N},
\end{aligned}
$$

where

$$
\begin{array}{ll}
\boldsymbol{a}_{1, Q}=\lambda_{1}\left(r \mathbf{1}^{(5)}-\boldsymbol{a}_{1, X}\right)-\dot{\boldsymbol{\lambda}}_{1} ; & \boldsymbol{b}_{1, Q}=\left(\boldsymbol{i}_{14}^{(1,6)}+\frac{\boldsymbol{k}_{1,(1, \cdot)}}{r+a_{G}}-\boldsymbol{\lambda}_{1} \boldsymbol{k}_{1, X}\right) \boldsymbol{\sigma}_{1, S S}^{1 / 2} \\
\boldsymbol{a}_{2, Q}=\lambda_{2}\left(r \mathbf{1}^{(3)}-\boldsymbol{a}_{2, X}\right)-\dot{\boldsymbol{\lambda}}_{2} ; & \boldsymbol{b}_{2, Q}=\left(\boldsymbol{i}_{13}^{(1,5)}+\frac{\boldsymbol{k}_{2,(1, \cdot)}}{r+a_{G}}-\boldsymbol{\lambda}_{2} \boldsymbol{k}_{2, X}\right) \boldsymbol{\sigma}_{2, S S}^{1 / 2} .
\end{array}
$$

$\boldsymbol{a}_{1, q}=\boldsymbol{i}_{12}^{(1,5)} ; \boldsymbol{b}_{1, q}=\boldsymbol{i}_{13}^{(1,6)} ; \boldsymbol{a}_{2, q}=i_{13}^{(1,3)} ; \boldsymbol{b}_{2, q}=\boldsymbol{i}_{12}^{(1,5)} ;$ and $d \boldsymbol{w}_{i, t}=\boldsymbol{\sigma}_{i, S S}^{-1 / 2}\left(d \boldsymbol{S}_{i, t}\right.$ $\left.\mathrm{E}_{i, t}\left[d \boldsymbol{S}_{i, t}\right]\right)(i=1,2)$. Note that $d \boldsymbol{w}_{i, t}$ behave as increments of a standard Wiener process everywhere except at $t=t_{k}$ where a jump occurs with a normal distribution as given by system (B4).

$\boldsymbol{X}_{i, t}$ governs the expected excess returns (on both the stock and private technology) faced by investor $i$. Given that $\boldsymbol{X}_{i, t}$ follows a Gaussian Markov process, it fully determines investor $i$ 's current and future investment opportunities. Also, $\boldsymbol{a}_{i, Q}$ and $\boldsymbol{b}_{i, Q}$ are periodic functions of time on $\mathcal{T}$, and $\boldsymbol{a}_{i, q}$ and $\boldsymbol{b}_{i, q}$ are constant matrices. Thus, the return process exhibits periodicity 
over time with periodicity $T+N$. Given equation (B7), investor $i$ 's control problem can be solved explicitly. We start by conjecturing that his value function has the following form:

$$
\begin{aligned}
t \in \mathcal{T}_{k}: \quad J_{i, t}=-\exp \left\{-\rho t-r \gamma W_{i, t}-\frac{1}{2} \boldsymbol{X}_{i, t}^{\prime} \boldsymbol{v}_{i} \boldsymbol{X}_{i, t}\right\}, \\
t \in \mathcal{N}_{k}: \quad J_{i, t}^{*}=-\exp \left\{-\rho t-r \gamma W_{i, t}-\frac{1}{2} \boldsymbol{X}_{i, t}^{* \prime} \boldsymbol{v}_{i} \boldsymbol{X}_{i, t}^{*}\right\},
\end{aligned}
$$

where $i=1,2, k=0,1, \ldots, W_{i, t}=W_{i, t}^{0}+\theta_{i, n_{k}} F_{i, t}, W_{i, t}^{0}$ is investor $i$ 's investment in the risk-free asset, $\boldsymbol{X}_{i, t}^{*}=\left[\boldsymbol{X}_{i, t}^{\prime}, \theta_{i, n_{k}}\right]^{\prime}, \boldsymbol{v}_{1}$ is a symmetric matrix $(5 \times 5$ for $t \in \mathcal{T}$ and $6 \times 6$ for $t \in \mathcal{N})$, and $\boldsymbol{v}_{2}$ is a symmetric matrix $(3 \times 3$ for $t \in \mathcal{T}$ and $4 \times 4$ for $t \in \mathcal{N})$. Define $m_{1}=6, m_{2}=4$, and $n_{i}=m_{i}-1$ for $i=1,2$. We show that the conjectured value function gives the solution to investor $i$ 's control problem by verifying that it satisfies the Bellman equation:

$$
\begin{aligned}
t \in \mathcal{T}_{k}: \quad 0= & \sup _{c_{i}, \theta_{i}, y_{i}}\left\{-e^{-\rho t-\gamma c_{i}}+\mathrm{E}_{i, t}\left[d J_{i, t}\right] / d t\right\} \\
& \text { s.t. } d W_{i, t}=\left(r W_{i, t}-c_{i, t}\right) d t+\theta_{i, t} d Q_{t}+y_{i, t} d q_{i, t} ; \\
t \in \mathcal{N}_{k}: \quad 0= & \sup _{c_{i}, y_{i}}\left\{-e^{-\rho t-\gamma c_{i}}+\mathrm{E}_{i, t}\left[d J_{i, t}^{*}\right] / d t\right\} \\
& \text { s.t. } d W_{i, t}^{0}=\left(r W_{i, t}^{0}-c_{i, t}\right) d t+\theta_{i, n_{k}} d D_{t}+y_{i, t} d q_{i, t} ;
\end{aligned}
$$

and the required boundary conditions. Define $\boldsymbol{a}_{i, W}=\left[\boldsymbol{a}_{i, Q} ; \boldsymbol{a}_{i, q}\right]$ and $\boldsymbol{b}_{i, W}=$ $\left[\boldsymbol{b}_{i, Q} ; \boldsymbol{b}_{i, q}\right]$. Substituting the conjectured form of the value function into the Bellman's equation and applying Itô's lemma, we obtain the following expression for the optimal policies:

$$
\begin{aligned}
t \in\left[t_{k}, n_{k}\right):\left[\begin{array}{l}
\boldsymbol{h}_{i, \theta} \\
\boldsymbol{h}_{i, y}
\end{array}\right] & =\frac{1}{r \gamma} \boldsymbol{\sigma}_{i, W W}^{-1}\left(\boldsymbol{a}_{i, W}-\boldsymbol{\sigma}_{i, W X} \boldsymbol{v}_{i}\right) \\
t=n_{k}:\left[\begin{array}{c}
\boldsymbol{h}_{i, \theta} \\
\boldsymbol{h}_{i, y}
\end{array}\right] & {\left[\begin{array}{l}
\boldsymbol{v}_{i,\left(m_{i}, m_{i}\right)}^{-1}\left(r \gamma \boldsymbol{\lambda}_{i}-\boldsymbol{v}_{i,\left(m_{i}, 1: n_{i}\right)}\right) \\
\frac{1}{r \gamma} \sigma_{q q}^{-1}\left\{\boldsymbol{a}_{i, q}-\boldsymbol{\sigma}_{i, q X}\left(\boldsymbol{v}_{i,\left(1: n_{i}, 1: n_{i}\right)}-\boldsymbol{v}_{i,\left(m_{i}, 1: n_{i}\right)}^{\prime} \boldsymbol{h}_{i, \theta}\right)\right.
\end{array}\right] } \\
t \in\left(n_{k}, t_{k+1}\right):\left[\begin{array}{c}
\left.-r \gamma \sigma_{i, q F} \boldsymbol{h}_{i, \theta}\right\} \\
h_{i, y}
\end{array}\right] & =\left[\begin{array}{c}
\frac{1}{r \gamma} \sigma_{q q}^{-1}\left(\boldsymbol{a}_{i, q}-\boldsymbol{\sigma}_{i, q X} \boldsymbol{v}_{i,\left(1: n_{i}, 1: n_{i}\right)}\right) \\
-\frac{1}{r \gamma} \sigma_{q q}^{-1}\left(r \gamma \sigma_{i, F q}-\boldsymbol{\sigma}_{i, q X} \boldsymbol{v}_{i,\left(1: n_{i}, 1: n_{i}\right)}^{\prime}\right)
\end{array}\right] .
\end{aligned}
$$


Next, define

$$
\begin{aligned}
& \boldsymbol{b}_{1, X}=\boldsymbol{k}_{1, X} ; \quad \boldsymbol{b}_{2, X}=\boldsymbol{k}_{2, X} ; \quad \overline{\boldsymbol{b}}_{2, F}=\left[\lambda_{G}\left(t_{k+1}\right),-\lambda_{1}\left(t_{k+1}\right)\right] \boldsymbol{o}_{2,(1: 2,1: 2)}^{1 / 2}\left(t_{k+1}^{-}\right) ; \\
& \boldsymbol{a}_{1, X}^{o}=\operatorname{diag}\left\{\mathbf{1}^{(3)}, \mathbf{1}^{(2)}-\boldsymbol{k}_{2,(1: 2)}\left(t_{k+1}\right)\left[\lambda_{G}\left(t_{k+1}\right),-\lambda_{1}\left(t_{k+1}\right)\right] \boldsymbol{o}_{2,(1: 2,1: 2)}^{1 / 2}\left(t_{k+1}^{-}\right)\right\} \\
& \boldsymbol{b}_{1, X}^{o}=\left[\mathbf{0} ; \mathbf{0} ; \boldsymbol{k}_{1,(3, \cdot)}\left(t_{k+1}\right) \boldsymbol{o}_{1,(3,3)}^{1 / 2}\left(t_{k+1}^{-}\right) ; \mathbf{0} ; \mathbf{0}\right] ; \\
& \boldsymbol{a}_{2, X}^{o}=\mathbf{1}^{(3)} ; \quad \boldsymbol{b}_{1, F}^{o}=\lambda_{2}\left(t_{k+1}\right) \boldsymbol{k}_{1,(3, \cdot)}\left(t_{k+1}\right) \boldsymbol{o}_{1,(3,3)}^{1 / 2}\left(t_{k+1}^{-}\right) ; \\
& \boldsymbol{b}_{2, X}^{o}=\left[\mathbf{0} ; \boldsymbol{k}_{2,(1: 2,)}\left(t_{k+1}\right)\left[\lambda_{G}\left(t_{k+1}\right) ;-\lambda_{1}\left(t_{k+1}\right)\right] \boldsymbol{o}_{2,(1: 2,1: 2)}^{1 / 2}\left(t_{k+1}^{-}\right)\right] \\
& \boldsymbol{b}_{2, F}^{o}=\left(\lambda_{G}\left(t_{k+1}\right) \boldsymbol{k}_{2,(1, \cdot)}\left(t_{k+1}\right)-\lambda_{1}\left(t_{k+1}\right) \boldsymbol{k}_{2,(2, \cdot)}\left(t_{k+1}\right)\right) \overline{\boldsymbol{b}}_{2, F}
\end{aligned}
$$

and

$$
\begin{aligned}
& \boldsymbol{\delta}_{i, 2}=\boldsymbol{a}_{i, X}^{o \prime} \boldsymbol{v}_{i}\left(t_{k+1}\right) \boldsymbol{b}_{i, X}^{o}\left[\mathbf{1}^{\left(n_{i}\right)}+\boldsymbol{b}_{i, X}^{o \prime} \boldsymbol{v}_{i}\left(t_{k+1}\right) \boldsymbol{b}_{i, X}^{o}\right]^{-1} \boldsymbol{b}_{i, X}^{o \prime} \boldsymbol{v}_{i}\left(t_{k+1}\right) \boldsymbol{a}_{i, X}^{o} ; \\
& \boldsymbol{\delta}_{i, 1}=\left[\boldsymbol{b}_{i, F}^{o}-\lambda_{i}\left(t_{k+1}\right) \boldsymbol{b}_{i, X}^{o}\right]\left[\mathbf{1}^{\left(n_{i}\right)}+\boldsymbol{b}_{i, X}^{o \prime} \boldsymbol{v}_{i}\left(t_{k+1}\right) \boldsymbol{b}_{i, X}^{o}\right]^{-1} \boldsymbol{b}_{i, X}^{o \prime} \boldsymbol{v}_{i}\left(t_{k+1}\right) \boldsymbol{a}_{i, X}^{o} ; \\
& \delta_{i, 0}=\boldsymbol{b}_{i, F}^{o}\left[\mathbf{1}^{\left(n_{i}\right)}+\boldsymbol{b}_{i, X}^{o \prime} \boldsymbol{v}_{i}\left(t_{k+1}\right) \boldsymbol{b}_{i, X}^{o}\right]^{-1} \boldsymbol{b}_{i, F}^{o \prime} .
\end{aligned}
$$

Substituting the optimal policies into the Bellman's equation, we obtain equality with the conjectured form when $\boldsymbol{v}_{i}$ satisfies the following:

$$
\begin{aligned}
& t=t_{k+1}: \quad \boldsymbol{v}_{i}\left(t_{k+1}^{-}\right)=\Delta^{o}\left[\boldsymbol{v}_{i}\left(t_{k+1}\right)\right] \\
& t \in\left(n_{k}, t_{k+1}\right): \quad \dot{\boldsymbol{v}}_{i}=\boldsymbol{v}_{i} \boldsymbol{\sigma}_{i, X^{*} X^{*}} \boldsymbol{v}_{i}-\left(\boldsymbol{a}_{i, q}-\boldsymbol{\sigma}_{i, q X^{*}} \boldsymbol{v}_{i}\right)^{\prime} \boldsymbol{\sigma}_{i, q q}^{-1}\left(\boldsymbol{a}_{i, q}-\boldsymbol{\sigma}_{i, q X^{*}} \boldsymbol{v}_{i}\right) \\
& +r \boldsymbol{v}_{i}+\left(\boldsymbol{a}_{i, X^{*}} \boldsymbol{v}_{i}+\boldsymbol{v}_{i} \boldsymbol{a}_{i, X^{*}}^{\prime}\right)-\left[\bar{v}+\operatorname{tr}\left(\boldsymbol{\sigma}_{i, X^{*} X^{*}} \boldsymbol{v}_{i}\right)\right] \boldsymbol{i}_{11} ; \\
& t=n_{k}: \quad \boldsymbol{v}_{i}\left(n_{k}\right)=\Delta^{c}\left[\boldsymbol{v}_{i}\left(n_{k}^{+}\right)\right] ; \\
& t \in\left[t_{k}, n_{k}\right]: \quad \dot{\boldsymbol{v}}_{i}=\boldsymbol{v}_{i} \boldsymbol{\sigma}_{i, X X} \boldsymbol{v}_{i}-\left(\boldsymbol{a}_{i, W}-\boldsymbol{\sigma}_{i, W X} \boldsymbol{v}_{i}\right)^{\prime} \boldsymbol{\sigma}_{i, W W}^{-1}\left(\boldsymbol{a}_{i, W}-\boldsymbol{\sigma}_{i, W X} \boldsymbol{v}_{i}\right) \\
& +r \boldsymbol{v}_{i}+\left(\boldsymbol{a}_{i, X} \boldsymbol{v}_{i}+\boldsymbol{v}_{i} \boldsymbol{a}_{i, X}^{\prime}\right)-\left[\bar{v}+\operatorname{tr}\left(\boldsymbol{\sigma}_{i, X X} \boldsymbol{v}_{i}\right)\right] \boldsymbol{i}_{11} ; \\
& \boldsymbol{v}_{i}\left(t_{k}\right)=\boldsymbol{v}_{i}\left(t_{k+1}\right)
\end{aligned}
$$

where

$$
\begin{aligned}
& \Delta^{o}\left(v_{i}\right)=\left[\begin{array}{cc}
\boldsymbol{a}_{i, X}^{o \prime} \boldsymbol{v}_{i} \boldsymbol{a}_{i, X}^{o}-\boldsymbol{\delta}_{i, 2} & r \gamma\left(\boldsymbol{\lambda}_{i} \boldsymbol{a}_{i, X}^{o}-\boldsymbol{\delta}_{i, 1}\right)^{\prime} \\
r \gamma\left(\boldsymbol{\lambda}_{i} \boldsymbol{a}_{i, X}^{o}-\boldsymbol{\delta}_{i, 1}\right) & (r \gamma)^{2} \delta_{i, 0}
\end{array}\right], \\
& \Delta^{c}\left(v_{i}\right)=\boldsymbol{v}_{i,\left(1: n_{i}, 1: n_{i}\right)}+\boldsymbol{v}_{i,\left(m_{i}, m_{i}\right)}^{-1}\left(r \gamma \boldsymbol{\lambda}-\boldsymbol{v}_{i,\left(m_{i}, 1: n_{i}\right)}\right)^{\prime}\left(r \gamma \boldsymbol{\lambda}-\boldsymbol{v}_{i,\left(m_{i}, 1: n_{i}\right)}\right),
\end{aligned}
$$

and $\bar{v}=2(\rho+r \ln r-r)$. 
To yield equation (22), define $\Delta \boldsymbol{v}_{i}\left(t_{k}\right) \equiv \boldsymbol{v}_{i}\left(t_{k}\right)-\boldsymbol{v}_{i}\left(t_{k}^{-}\right)$and $\Delta \boldsymbol{v}_{i}\left(n_{k}\right) \equiv$ $\boldsymbol{v}_{i}\left(n_{k-1}^{+}\right)-\boldsymbol{v}_{i}\left(n_{k-1}\right)$, and $\boldsymbol{g}_{i, v}$ as follows:

$$
\boldsymbol{g}_{i, v} \equiv \dot{\boldsymbol{v}}_{i}+\sum_{k=1}^{\infty}\left\{\delta_{D}\left(t-t_{k}\right) \Delta \boldsymbol{v}_{i}\left(t_{k}\right)+\delta_{D}\left(t-n_{k-1}^{+}\right) \Delta \boldsymbol{v}_{i}\left(n_{k}\right)\right\}
$$

A periodic solution to equation (22) is given by the initial value $\boldsymbol{v}_{i}\left(t_{k+1}\right)$ that equals $\boldsymbol{v}_{i}\left(t_{k}\right)$ from equation (22) as equation (B11e) requires.

We now prove the existence of a periodic solution to equation (22) assuming that $\lambda$ is periodic on $\mathcal{T}$ and $\boldsymbol{o}$ is periodic on $\mathcal{T} \cup \mathcal{N}$. This is accomplished through the following two lemmas. The first says that equations (B11d) and (B11b) have solutions; the second says that a periodic solution exists. For convenience, define $\boldsymbol{v}_{i}^{*}=\boldsymbol{v}_{i,\left(1: n_{i}, 1: n_{i}\right)}, \boldsymbol{u}_{i}^{*}=\boldsymbol{v}_{i,\left(m_{i}, 1: n_{i}\right)}$, and $u_{i, 0}^{*}=\boldsymbol{v}_{i,\left(m_{i}, m_{i}\right)}$.

Lemma B.2: There exist $\alpha_{i}, \beta_{i}, \alpha_{i}^{*}$, and $\beta_{i}^{*}$ all positive with $\alpha_{i}, \alpha_{i}^{*}<1$ such that (a) given the terminal value $\boldsymbol{v}_{i, n_{k}}$, which is symmetric and semipositive definite, equation (B11d) has a solution $\boldsymbol{v}_{i}\left(t ; \boldsymbol{v}_{i, n_{k}}\right)$ which is also symmetric, positive semidefinite, and $\left\|\boldsymbol{v}_{i}\left(t ; \boldsymbol{v}_{i, n_{k}}\right)\right\| \leq \alpha_{i}\left\|\boldsymbol{v}_{i, n_{k}}\right\|+\beta_{i}$; and (b) given the terminal value $\boldsymbol{v}_{i, t_{h+1}}$, which is symmetric and semipositive definite, equation (B11b) has a solution, $\boldsymbol{v}_{i}^{*}\left(t ; \boldsymbol{v}_{i, t_{h+1}^{*}}^{*}\right)$, which is symmetric, positive semidefinite, and $\left\|\boldsymbol{v}_{i}^{*}\left(t ; \boldsymbol{v}_{i, t_{k+1}}^{*}\right)\right\| \leq \alpha_{i}^{*}\left\|\boldsymbol{v}_{i, t_{k+1}}^{*}\right\|+\beta_{i}^{*}$.

Proof: First, we verify this claim for equation (B11d). Using the notation of Lemma A.3, let $\boldsymbol{r}=\boldsymbol{v}_{i}, \boldsymbol{a}_{0}=\boldsymbol{a}_{i, W}^{\prime} \boldsymbol{\sigma}_{i, W W}^{-1} \boldsymbol{a}_{i, W}, \boldsymbol{a}_{1}=-\frac{1}{2} r \mathbf{1}^{\left(n_{i}\right)}-\boldsymbol{a}_{i, X}-$ $\boldsymbol{a}_{i, W}^{\prime} \boldsymbol{\sigma}_{i, W W}^{-1} \boldsymbol{\sigma}_{i, W X}, \quad \boldsymbol{a}_{2}=\boldsymbol{\sigma}_{i, X X}-\boldsymbol{\sigma}_{i, W X}^{\prime} \boldsymbol{\sigma}_{i, W W}^{-1} \boldsymbol{\sigma}_{i, W X}$, and $\boldsymbol{a}_{3}(\mathrm{t}, \boldsymbol{r})=[\bar{v}+$ $\left.\operatorname{tr}\left(\boldsymbol{\sigma}_{i, X X} \boldsymbol{r}\right)\right] \boldsymbol{i}_{11}$. It is easy to verify that $\boldsymbol{a}_{0}$ and $\boldsymbol{a}_{2}$ are positive definite. Assume $\bar{v} \geq 0$ (this lemma can easily be shown to hold for $\bar{v}<0$ ). Since trace is a linear operator, $\boldsymbol{a}_{3}(t, \boldsymbol{r})$ is a linear positive operator. Hence, equation (B11d) is a matrix Riccati differential equation. By Lemma A.3, given $\boldsymbol{v}_{i}\left(n_{k}\right)=\boldsymbol{v}_{i, n_{k}}>0, \boldsymbol{v}_{i}\left(t ; \boldsymbol{v}_{i, n_{k}}\right)$ exists and is symmetric, positive semidefinite. Let $\boldsymbol{m}=-\left(\boldsymbol{a}_{i, W}^{\prime} \boldsymbol{\sigma}_{i, W W}^{-1} \boldsymbol{\sigma}_{i, W X}\right)$, then by Lemma A.3, $\boldsymbol{v}_{i}\left(t ; \boldsymbol{v}_{i, n_{k}}\right) \leq \boldsymbol{e}\left(t ; \boldsymbol{v}_{i, n_{k}}\right)$, where $\boldsymbol{e}$ is the solution to a linear system given in Lemma A.3 with $\boldsymbol{a}_{1}-\boldsymbol{m}=-\frac{1}{2} r \mathbf{1}^{\left(n_{i}\right)}-\boldsymbol{a}_{i, X}<0$. It follows then that $\left\|\boldsymbol{e}\left(t ; \boldsymbol{v}_{i, n_{k}}\right)\right\| \leq \alpha_{i}\left\|\boldsymbol{v}_{i, n_{k}}\right\|+$ $\beta_{i}$, where

$$
\begin{aligned}
& \alpha_{i}=\exp \left\{-\left(r+2 a_{Y}\right) T\right\} ; \quad \boldsymbol{\zeta}_{i}=\exp \left\{\left[-\frac{1}{2} r \mathbf{1}^{\left(n_{i}\right)}-\boldsymbol{a}_{i, X}\right](T-s)\right\} ; \\
& \beta_{i}=\left\|\int_{0}^{T} \zeta_{i}\left[\boldsymbol{m}(T-s)^{\prime} \boldsymbol{a}_{2}^{-1} \boldsymbol{m}(T-s)+\boldsymbol{a}_{3}(T-s)+\boldsymbol{a}_{0}(T-s)\right] \zeta_{i} d s\right\| .
\end{aligned}
$$

Next, consider equation (B11b). Let $\boldsymbol{r}=\boldsymbol{v}_{i}^{*}, \boldsymbol{a}_{0}=\boldsymbol{a}_{i, q}^{\prime} \sigma_{i, q q}^{-1} \boldsymbol{a}_{i, q}, \boldsymbol{a}_{1}=-\frac{1}{2} r \mathbf{1}^{\left(n_{i}\right)}-$ $\boldsymbol{a}_{i, X}, \boldsymbol{a}_{2}=\boldsymbol{\sigma}_{i, X X}$, and $\boldsymbol{a}_{3}(t, \boldsymbol{r})=\left[\bar{v}+\operatorname{tr}\left(\boldsymbol{\sigma}_{i, X X} \boldsymbol{r}\right)\right] \boldsymbol{i}_{11}$. Apply the same reasoning as above and equation (B11b) is a matrix Riccati equation as well. Let 
$\boldsymbol{v}_{i}^{*}\left(t_{k+1}\right)=\boldsymbol{v}_{i, t_{k+1}}^{*}>0$ and $\boldsymbol{m}=\mathbf{1}^{\left(n_{i}\right)}$, then it follows from the above reasoning that $\left\|\boldsymbol{v}_{i}^{*}\left(t ; \boldsymbol{v}_{i, t_{k+1}}^{*}\right)\right\| \leq \alpha_{i}^{*}\left\|\boldsymbol{v}_{i, t_{k+1}}^{*}\right\|+\beta_{i}^{*}$ where

$$
\begin{aligned}
& \alpha_{i}^{*}=\exp \left\{-\left(r+2 a_{Y}\right) T\right\} ; \quad \boldsymbol{\zeta}_{i}^{*}=\exp \left\{\left[-\frac{1}{2} r \mathbf{1}^{\left(n_{i}\right)}-\boldsymbol{a}_{i, X}\right](T-s)\right\} \\
& \beta_{i}^{*}=\left\|\int_{0}^{T} \boldsymbol{\zeta}_{i}^{*}\left\{\boldsymbol{m}(T-s)^{\prime} \boldsymbol{a}_{2}^{-1} \boldsymbol{m}(T-s)+\boldsymbol{a}_{3}(T-s)+\boldsymbol{a}_{0}(T-s)\right\} \zeta_{i}^{*} d s\right\| .
\end{aligned}
$$

Finally, given $\boldsymbol{v}_{i}^{*}\left(t ; \boldsymbol{v}_{i, t_{k+1}}^{*}\right), \boldsymbol{u}_{i, t_{k+1}}^{*}$, and $u_{i 0, t_{k+1}}^{*}, \boldsymbol{u}_{i}^{*}$, and $u_{i, 0}^{*}$ exist since they satisfy linear differential systems.

LeMma B.3: The system (B11) has a solution.

Proof: For $i=1,2$, let $\boldsymbol{z}_{i}=\left[\boldsymbol{v}_{i}\right]$ for $t \in \mathcal{T}, \boldsymbol{z}_{i}=\left[\left[\boldsymbol{v}_{i}^{*}\right]^{\prime},\left[\boldsymbol{u}_{i}^{*}\right]^{\prime},\left[u_{i, 0}^{*}\right]\right], t \in \mathcal{N}$, $\boldsymbol{z}_{a}=\boldsymbol{z}_{i}$. First, the system conforms to the general boundary value problem, equation (A5). Next, since $\sigma_{i, X X}-\operatorname{diag}\left\{0,0,0,\left(\sigma_{q X}\right)^{2} / \sigma_{q q}\right\}>0$, $\left\|\boldsymbol{u}_{i}^{*}\left(t ; \boldsymbol{v}_{i}\left(t ; \boldsymbol{v}_{i, n_{k}}\right)\right)\right\| \leq \beta$ where

$$
\begin{aligned}
& \zeta=\exp \left\{-\frac{1}{2}\left(r+2 a_{Y}\right)(N-t)\right\}\left(\left\|r \gamma \boldsymbol{\lambda}\left(t_{k+1}\right)\right\|+\left\|\boldsymbol{b}_{i, F}\right\|\right), \\
& \beta=\zeta+\left\|\int_{0}^{N} \exp \left\{-\left(r+2 a_{Y}\right)(N-s)\right\}\left\{r \gamma\left(\sigma_{i, F q} \sigma_{q q}^{-1} \boldsymbol{a}_{i, q}+\boldsymbol{\sigma}_{i, F X}\right)\right\} d s\right\| .
\end{aligned}
$$

Thus, $\boldsymbol{u}_{i}^{*}\left(n_{k} ; \boldsymbol{v}_{i}\left(t ; \boldsymbol{v}_{i, n_{k}}\right)\right)$ is uniformly bounded in $\boldsymbol{v}_{i}\left(t ; \boldsymbol{v}_{i, n_{k}}\right)$. Here, $\alpha_{\mathrm{i}}, \alpha_{i}^{*}, \beta_{i}$, and $\beta_{i}^{*}$ are given in Lemma B.2. Let $\zeta_{i}=\alpha_{i}^{*} \beta_{i}+\beta_{i}^{*}+\beta$ and $\eta_{i}=\alpha_{i}^{*} \alpha_{i}$. Obviously, $\eta_{\mathrm{i}}<1$. Let $L_{i}$ be the space of symmetric, positive semidefinite matrices such that their norms are less than $\psi_{i}$ where $\psi_{i}=\zeta_{i} /\left(1-\eta_{i}\right)$. Let $M_{i}: L_{i} \rightarrow L_{i}$ be given by the right-hand side of equation (B11c). $L_{i}$ is a nonempty, closed, bounded convex subset of a finite-dimensional normed vector space. $M_{i}$ is symmetric since the right-hand side of equation (B11c) is symmetric. $M_{i} \geq 0$ because $u_{i, 0}^{*}\left(n_{k}\right)>0$ is positive given its terminal value defined in equation (B11a) and $\boldsymbol{v}_{i}^{*}\left(n_{k}\right) \geq 0$ by Lemma B.2. It is easy to show that $\left\|M_{i}\left(\boldsymbol{v}_{i, n_{k}}\right)\right\| \leq \psi_{i} /\left(1-\eta_{i}\right)$. By Lemma A.1, the result follows.

A solution to system (B11) gives a solution to the Bellman equation under the desired boundary condition. This completes our proof of Lemma 2. Q.E.D.

\section{Appendix C. Proof of Theorem 1}

We have shown that (a) for a conjectured periodic price function of the form in equation (18), a periodic solution to equation (16b) exists for investor $i$, and (b) given the periodic price function and a periodic solution for $\boldsymbol{o}_{i}(t)$, a periodic solution to the control problem of investor $i$ exists. Now we want to show that an equilibrium with the conjectured price function exists for $\omega$ close to one. We do this in two steps. First, we show that for $\omega=1$, there exists a periodic price function that satisfies equation (25) in which the hold- 
ings of each investor are derived from the periodic solutions to equations (16b) and (22). Then, we verify that our system satisfies conditions stated in Lemma A.2 and Lemma A.4, which concludes the proof.

Given the investors' optimal investment policies, the market clearing condition then becomes

$$
\omega \lambda_{1} \boldsymbol{h}_{1, \theta}^{\prime}+(1-\omega) \boldsymbol{\tau}^{\prime} \boldsymbol{h}_{2, \theta}^{\prime}=\boldsymbol{i}_{11}^{(5,1)} \quad \forall t \in \mathcal{T}_{k}, k=0,1, \ldots,
$$

where $\boldsymbol{\tau}=\left[\left[\lambda_{1}, 0,0,0,0\right] ;\left[0, \lambda_{1}, 0,-\lambda_{G}, 0\right] ;\left[0,0, \lambda_{1}, 0,0\right]\right]$. The periodic condition for the price function requires for $k=0,1, \ldots$,

$$
\boldsymbol{\lambda}_{i}\left(t_{k+1}\right)=\boldsymbol{\lambda}_{i}\left(t_{k}\right)
$$

Let $\boldsymbol{z}_{i}=\left[\boldsymbol{v}_{i}\right]$ for $t \in \mathcal{T}, \boldsymbol{z}_{i}=\left[\left[\boldsymbol{v}_{i}^{*}\right] ;\left[\boldsymbol{u}_{i}^{*}\right] ; u_{i, 0}^{*}\right], t \in \mathcal{N}, i=1,2$. Let $\boldsymbol{z}_{a}=\left[\boldsymbol{\lambda}^{\prime} ; \boldsymbol{z}_{1} ; \boldsymbol{z}_{2}\right]$ for $t \in \mathcal{T}$ and $\boldsymbol{z}_{a}=\left[\boldsymbol{z}_{1} ; \boldsymbol{z}_{2}\right]$ for $t \in \mathcal{N}$. Let $\boldsymbol{z}_{b}=\left[\boldsymbol{o}_{1} ; \boldsymbol{o}_{2}\right]$. Then the problem conforms to boundary value problem, equation (A5). Let $\omega_{0}=1$. Existence of $\boldsymbol{z}\left(t, \theta, \omega_{0}\right)$ is given by Proposition 1 (see Appendix D), Lemma B.3, and Lemma B.1. We first note that in the case of asymmetric information, $\dot{z}$ and $\boldsymbol{z}$ are related implicitly: $0=\boldsymbol{F}(\dot{\boldsymbol{z}}, \boldsymbol{z} ; \theta, \omega), \forall t \in\left[t_{k}, n_{k}\right]$. By the Implicit Function Theorem (see, e.g., Protter and Morrey (1991)), $\boldsymbol{F}$ defines an implicit function: $\dot{\boldsymbol{z}}=\boldsymbol{f}(\boldsymbol{z} ; \theta, \omega)$ if $\nabla_{\dot{z}} \boldsymbol{F}$ is nonsingular. For $\omega_{0}=1$ and $\theta_{0}=[0.001,1000,1 /$ $4,1 / 2,1,1,7,7,1,1 / 2,0,1 / 2,1 / 2], \operatorname{det}\left(\nabla_{\dot{z}} \boldsymbol{F}\right) \neq 0$. By Lemma A.4, $\dot{\boldsymbol{z}}=\boldsymbol{f}(\boldsymbol{z} ; \theta, \omega)$ exists generically. It remains to verify that $\boldsymbol{z}\left(t ; \theta, \omega_{0}\right)$ is an isolated solution. This is equivalent to showing that $\boldsymbol{m}\left(\theta, \omega_{0}\right)=\nabla_{z} \boldsymbol{g}\left(\boldsymbol{z}_{0}\right)+\nabla_{z} \boldsymbol{g}\left(\boldsymbol{z}_{T}\right) \exp \left\{\int_{t_{k}}^{n_{k}} \nabla_{z} \boldsymbol{f}\right\}$ is nonsingular (see Keller $\left(1992\right.$, p. 191)). Clearly, $\boldsymbol{m}\left(\theta, \omega_{0}\right)$ is analytic. It is easy to show that $\operatorname{det}\left[\boldsymbol{m}\left(\theta_{0}, \omega_{0}\right)\right] \neq 0$. By Lemma A.4, $\mathbf{m}\left(\theta, \omega_{0}\right)$ is generically nondegenerate. By Lemma A.2, Theorem 1 holds. Q.E.D.

\section{Appendix D. Proofs of Propositions 1-4}

Under symmetric information, the state vectors of investors are given by $\boldsymbol{X}_{1, t}=\boldsymbol{X}_{2, t}=\left[1, Y_{1, t}, Y_{2, t}\right]^{\prime}$. So, using the notation in the proof of Lemma 3, we now have $\boldsymbol{v}_{i}$ is $3 \times 3$ for $t \in \mathcal{T}$ and $4 \times 4$ during the night; $\boldsymbol{v}_{i}^{*}=\boldsymbol{v}_{i,\left(1: n_{i}, 1: n_{i}\right)}$, $\boldsymbol{u}_{i}^{*}=\boldsymbol{v}_{i,\left(m_{i}, 1: n_{i}\right)}$, and $u_{i, 0}^{*}=\boldsymbol{v}_{i,\left(m_{i}, m_{i}\right)}$, where $m_{i}=4$ for $i=1,2$ and $n_{i}=3$ for $i=1,2$.

Proof of Proposition 1: When $\kappa_{12}=1, Y_{1, t}=Y_{2, t}=Y_{t}$, and $\theta_{i, t}=1, i=1,2$. Thus $P_{t}=F_{t}-\left(\lambda_{0}+\lambda Y_{t}\right)$ and $\lambda_{0}$ and $\lambda$ are constants. Given that the two investors are identical, we can drop the index $i$. Let $\tilde{\boldsymbol{v}}$ equal $\boldsymbol{v}$ for $t \in \mathcal{T}$ and $\boldsymbol{v}^{*}$ for $t \in \mathcal{N}$. Equation (B11d) then reduces to the following:

$$
\begin{aligned}
& 0=r \tilde{\boldsymbol{v}}-\left(\boldsymbol{a}_{X} \tilde{\boldsymbol{v}}+\tilde{\boldsymbol{v}} \boldsymbol{a}_{X}^{\prime}\right)+\tilde{\boldsymbol{v}} \boldsymbol{\sigma}_{X X} \tilde{\boldsymbol{v}}-\frac{1}{\sigma_{q}^{2}} \boldsymbol{a}_{1, q}^{\prime} \boldsymbol{a}_{1, q}-\overline{\boldsymbol{v}} \boldsymbol{i}_{11} \\
& \boldsymbol{u}^{*}=r \gamma \lambda ; \quad u_{0}^{*}=r \gamma^{2}\left(\bar{\sigma}_{F}^{2}+\lambda^{2} \sigma_{Y}^{2}\right)
\end{aligned}
$$


where $\boldsymbol{\lambda}=\left[\lambda_{0}, \lambda, \lambda\right]$ and $\overline{\boldsymbol{v}}=2(\rho-r \log r-r)$. Given $\boldsymbol{\lambda}$, the equation for $\tilde{\boldsymbol{v}}$ has only two roots, one positive and one negative. The positive root corresponds to the optimal solution of the investors' control problem since it gives higher expected utility. $\lambda_{0}^{*}$ and $\lambda^{*}$ can then be solved from $\theta_{i, t}=1$. Q.E.D.

Proof of Proposition 2: From Proposition 1, it is easy to show that $\boldsymbol{u}_{1}^{*}=$ $r \gamma[0, \bar{\lambda}, 0], \boldsymbol{u}_{2}^{*}=r \gamma[0,0, \bar{\lambda}]$, and $u_{0}^{*}=r \gamma^{2}\left[\bar{\sigma}_{F}^{2}+(\bar{\lambda})^{2} \sigma_{Y}^{2}\right]$. Next $h(T)=-\bar{\lambda} / \bar{\lambda}_{0}$. Given the definition of $\bar{h}$ stated in Proposition 2 and $\bar{\lambda}$ and $\bar{\lambda}_{0}$ from Proposition 1 , it is straightforward to show that $\bar{\lambda} / \bar{\lambda}_{0}<|\bar{h}|$. Next, $\lambda_{0}$ is given by $r \lambda_{0}-\dot{\lambda}_{0}=r \gamma \bar{\sigma}_{F}^{2}$, with $\lambda_{0}(T)=\bar{\lambda}_{0}$, which has the solution $\lambda_{0}(t)=$ $\left(\bar{\lambda}_{0}-\gamma \bar{\sigma}_{F}^{2}\right) e^{-r(T-t)}+\gamma \bar{\sigma}_{F}^{2} \cdot \lambda_{0}(\mathrm{t})$ is strictly increasing over time. Q.E.D.

Proof of Proposition 3: Given the periodicity in equilibrium, $\mathrm{E}\left[P_{t_{h}}\right]=$ $-\lambda_{0}(0)$ and $\mathrm{E}\left[P_{n_{k}}\right]=\lambda_{0}(T)$, independent of $k=0,1, \ldots$ Thus $\mathrm{E}\left[R^{o o}\right]=$ $\mathrm{E}\left[R^{c c}\right]=0$. Furthermore, $\mathrm{E}\left[R^{c o}\right]=\lambda_{0}(T)-\lambda_{0}(0)=-\mathrm{E}\left[R^{o c}\right]$. For $\lambda_{0}(0)<$ $\lambda_{0}(T)$, we have $\mathrm{E}\left[R^{c o}\right]>0>\mathrm{E}\left[R^{o c}\right]$. Q.E.D.

Proof of Proposition 4: Since $P_{t}=F_{t}-\lambda_{0}(t)-\lambda(t) Y_{t}$ for $t \in \mathcal{T}$, we have

$$
R_{t, s}=\int_{t}^{s} d D_{\tau} d \tau+\left(F_{s}-F_{t}\right)-\left[\lambda(s) Y_{s}-\lambda(t) Y_{t}\right]
$$

Note that $Y_{s}=e^{-a_{Y}(s-t)} Y_{t}+\int_{t}^{s} e^{-a_{Y}(s-\tau)} \boldsymbol{b}_{Y} d \boldsymbol{w}_{\tau}$. It then follows that var$\left[R^{o o}\right]-\operatorname{var}\left[R^{c c}\right]=\left[\lambda^{2}(0)-\lambda^{2}(T)\right] \operatorname{var}\left[Y_{T}-Y_{0}\right]>0$ for $\lambda(0)>\lambda(T)$ and $\operatorname{var}\left[R^{o c}\right]-\operatorname{var}\left[R^{c o}\right]=0$. Q.E.D.

\section{Appendix E. Numerical Procedure}

We briefly discuss the numerical procedure used to solve for the periodic equilibrium. We use the Newton-Kantorovich method to solve this problem numerically (see, e.g., Kubicek (1983)). This recursive method linearizes the system and the boundary conditions around a conjectured solution to the nonlinear problem at a discrete number of points in the interval $\left[t_{k}, n_{k}\right]$. Since the system is linearized, it is easy to calculate an updated solution that satisfies the linearized system and boundary conditions from the conjectured solution. The updated solution is then used as the conjectured solution to start the next recursion. It can be shown that the limit of this recursion converges to the solution of the nonlinear problem given that the initial conjectured solution is not too far away from the true solution.

This method requires a sufficiently accurate initial guess of the true solution. We obtain such a guess by starting the recursion at $\omega=1$ for any given set of parameters since a solution exists at $\omega=1$. In order to calculate a solution at $\omega_{0}<1$, we begin by using the solution at $\omega=1$ as the initial guess to find a solution for an $\omega$ close to one and we repeat the same procedure to move toward $\omega_{0}$. 
Since we have no knowledge on the uniqueness of the solution, the above procedure also guarantees that we stay on the same branch of solutions. In particular, the solution gives the expected results when we take the limit $\omega \rightarrow 1$. We have also checked the solution by taking other limits in the parameter space such as $\sigma_{G} \rightarrow 0$ and have obtained the expected results.

\section{REFERENCES}

Admati, Anat R., and Paul Pfleiderer, 1988, A theory of intraday patterns: Volume and price variability, Review of Financial Studies 1, 3-40.

Admati, Anat R., and Paul Pfleiderer, 1989, Divide and conquer: A theory of intraday and day of the week mean effects, Review of Financial Studies 1, 189-223.

Ahlfors, Lars V., 1979, Complex Analysis (McGraw-Hill Book Co., New York).

Amihud, Yakov, and Haim Mendelson, 1987, Trading mechanisms and stock returns: An empirical investigation, Journal of Finance 42, 533-553.

Amihud, Yakov, and Haim Mendelson, 1991, Volatility, efficiency and trading: Evidence from the Japanese stock market, Journal of Finance 46, 1765-1791.

Andersen, Torben, and Tim Bollerslev, 1997, Intraday periodicity and volatility persistence in financial markets, Journal of Empirical Finance 4, 115-158.

Barclay, Michael J., Robert H. Litzenberger, and Jerold B. Warner, 1990, Private information, trading volume, and stock return variances, Review of Financial Studies 3, 233-253.

Border, Kim, 1985, Fixed Point Theorems with Applications to Economics and Game Theory (Cambridge University Press, Cambridge, U.K.).

Brock, William A., and Allan W. Kleidon, 1992, Periodic market closure and trading volume: A model of intraday bids and asks, Journal of Economic Dynamics and Control 16, 451-489.

Cao, Charles, Hyuk Choe, and Frank Hatheway, 1995, What is special about the opening? Evidence from Nasdaq, Working paper, Pennsylvania State University.

Campbell, John Y., and Albert S. Kyle, 1993, Smart money, noise trading, and stock price behavior, Review of Economic Studies 60, 1-34.

Chan, K. C., Wai-Ming Fong, Bong-Chan Kho, and René M. Stulz, 1996, Information, trading and stock returns: Lessons from dually-listed securities, Journal of Banking \& Finance 20, 1161-1187.

Cronin, Jane, 1994, Differential Equations: Introduction and Qualitative Theory (Marcel Dekker, New York).

Doob, Joseph L., 1991, Measure Theory (Springer-Verlag, New York).

Fama, Eugene F., 1965, The behavior of stock market prices, Journal of Business 38, 34-105.

Foster, F. Douglas, and S. Viswanathan, 1990, A theory of interday variations in volume, variance, and trading costs in securities markets, Review of Financial Studies 3, 593-624.

Foster, F. Douglas, and S. Viswanathan, 1993, The effect of public information and competition on trading volume and price volatility, Review of Financial Studies 6, 23-56.

French, Kenneth R., 1980, Stock returns and the weekend effect, Journal of Financial Economics 8, 55-69.

French, Kenneth R., and Richard Roll, 1986, Stock return variances: The arrival of information and the reaction of traders, Journal of Financial Economics 17, 5-26.

Gerety, Mason S., and Harold Mulherin, 1994, Price formation on stock exchanges: The evolution of trading within the day, Review of Financial Studies 7, 609-629.

Gibbons, Michael R., and Patrick Hess, 1981, Day of the week effects and asset returns, Journal of Business 54, 579-596.

Grossman, Sanford J., 1976, On the efficiency of competitive stock markets where traders have diverse information, Journal of Finance 31, 573-585.

Grossman, Sanford J., 1981, An introduction to the theory of rational expectations under asymmetric information, Review of Economic Studies 48, 541-559.

Harris, Lawrence, 1986, A transaction data study of weekly and intradaily patterns in stock returns, Journal of Financial Economics 16, 99-117. 
Harris, Lawrence, 1988, Intraday stock return patterns; in Elroy Dimson, ed.: Stock Market Anomalies (Cambridge University Press, Cambridge, U.K.).

Harris, Lawrence, 1989, A day-end transaction price anomaly, Journal of Financial and Quantitative Analysis 24, 29-45.

Harrison, J. Michael, and Stanley R. Pliska, 1981, Martingales and stochastic integrals in the theory of continuous trading, Stochastic Process Applications 11, 215-260.

He, Hua, and Jiang Wang, 1995, Differential information and dynamic behavior of stock trading volume, Review of Financial Studies 8, 919-972.

Hong, Harrison, and Jiang Wang, 1998, Trading and returns under periodic market closures, Working paper, MIT Sloan School of Management.

Huang, Chi-fu, 1985, Information structure and equilibrium asset prices, Journal of Economic Theory 35, 33-71.

Huang, Jennifer, and Jiang Wang, 1997, Market structure, security prices and informational efficiency, Macroeconomic Dynamics 1, 169-205.

Jain, Prem, and Gun-Ho Joh, 1988, The dependence between hourly prices under transactions and return uncertainty, Journal of Financial and Quantitative Analysis 23, 269-283.

Jazwinski, Andrew H., 1970, Stochastic Processes and Filtering Theory (Academic Press, New York).

Karatzas, Ioannis, and Steven E. Shreve, 1988, Brownian Motion and Stochastic Calculus (Springer, New York).

Keim, Donald, and Robert Stambaugh, 1984, A further investigation of weekend effect in stock returns, Journal of Finance 39, 819-835.

Keller, Herbert B., 1992, Numerical Methods for Two-point Boundary Value Problems (Dover Publications, New York).

Kleidon, Allan, and Ingrid Werner, 1996, U.K. and U.S. trading of British cross-listed stocks: An intraday analysis of market integration, Review of Financial Studies 9, 619-644.

Kubicek, Milan, 1983, Numerical Solution of Nonlinear Boundary Value Problems with Applications (Prentice-Hall, Englewood Cliffs, N.J).

Kyle, Albert, 1985, Continuous auctions and insider trading, Econometrica 53, 1315-1335.

Lighthill, M. James, 1958, Introduction to Fourier Analysis and Generalized Functions (Cambridge University Press, Cambridge, U.K.).

Liptser, Robert S., and Albert N. Shiryayev, 1977, Statistics of Random Processes, Volume I (Springer-Verlag, New York).

Liptser, Robert S., and Albert N. Shiryayev, 1978, Statistics of Random Processes, Volume II (Springer-Verlag, New York).

Lockwood, Larry J., and Scott C. Linn, 1990, An examination of stock market return volatility during overnight and intraday periods, 1964-1989, Journal of Finance 45, 591-601.

Merton, Robert C., 1969, Lifetime portfolio selection under uncertainty: The continuous time case, Review of Economics and Statistics 51, 247-257.

Merton, Robert C., 1971, Optimal consumption and portfolio rules in a continuous time model, Journal of Economic Theory 3, 373-413.

Merton, Robert C., 1990, Continuous-Time Finance (Blackwell, Oxford, U.K.).

Oldfield, George S., and Richard J. Rogalski, 1980, A theory of common stock returns over trading and non-trading periods, Journal of Finance 35, 729-751.

Protter, Murray H., and Charles B. Morrey, 1991, A First Course in Real Analysis (SpringerVerlag, New York).

Rogalski, Richard, 1984, New findings regarding day-of-the-week returns over trading and nontrading periods: A note, Journal of Finance 35, 1603-1614.

Rubinstein, Mark, 1974, An aggregation theorem for security markets, Journal of Financial Economics 3, 201-224.

Slezak, Steve L., 1994, A theory of the dynamics of security returns around market closures, Journal of Finance 49, 1163-1212.

Smirlock, Michael, and Laura Starks, 1986, Day-of-the-week and intraday effects in stock returns, Journal of Finance 35, 197-210.

Spiegel, Matthew, and Avanidhar Subrahmanyam, 1995, On intraday risk premium, Journal of Finance 50, 319-340. 
Stoll, Hans, and Robert Whaley, 1990, Stock market structure and volatility, Review of Financial Studies 3, 37-71.

Vayanos, Dimitri, 1999, Strategic trading and welfare in a dynamic market, Review of Economic Studies 66, 219-254.

Wang, Jiang, 1993, A model of intertemporal asset prices under asymmetric information, Review of Economic Studies 60, 249-282.

Wang, Jiang, 1994, A model of competitive stock trading volume, Journal of Political Economy $102,127-168$.

Wonham, William M., 1968, On a matrix Riccati equation of stochastic control, SIAM Journal of Control 6, 681-697.

Wood, Robert A., Thomas H. McInish, and J. Keith Ord, 1985, An investigation of transaction data for NYSE stocks, Journal of Finance 40, 723-741. 